University of Louisville

ThinkIR: The University of Louisville's Institutional Repository

Electronic Theses and Dissertations

$8-2020$

\title{
Trust in primary care providers: perspectives from deaf people.
}

Greir Ander Huck Flynn

University of Louisville

Follow this and additional works at: https://ir.library.louisville.edu/etd

Part of the Family Practice Nursing Commons

\section{Recommended Citation}

Flynn, Greir Ander Huck, "Trust in primary care providers: perspectives from deaf people." (2020).

Electronic Theses and Dissertations. Paper 3491.

https://doi.org/10.18297/etd/3491

This Doctoral Dissertation is brought to you for free and open access by ThinkIR: The University of Louisville's Institutional Repository. It has been accepted for inclusion in Electronic Theses and Dissertations by an authorized administrator of ThinkIR: The University of Louisville's Institutional Repository. This title appears here courtesy of the author, who has retained all other copyrights. For more information, please contact thinkir@louisville.edu. 
TRUST IN PRIMARY CARE PROVIDERS: PERSPECTIVES FROM DEAF PEOPLE

By

Greir Ander Huck Flynn

Bachelor of Science in Human Biology, Indiana University - Bloomington, IN, 2010

Bachelor of Science in Nursing, Bellarmine University - Louisville, KY, 2012

Master of Science in Nursing, Bellarmine University - Louisville, KY, 2015

A Dissertation

Submitted to the Faculty of the School of Nursing of the University of Louisville

In Partial Fulfillment of the Requirements

For the Degree of

Doctor of Philosophy in Nursing

School of Nursing

University of Louisville

Louisville, Kentucky

August 2020 
Copyright 2020 by Greir Ander Huck Flynn

All rights reserved 

TRUST IN PRIMARY CARE PROVIDERS: PERSPECTIVES FROM DEAF PEOPLE

By

Greir Ander Huck Flynn

Bachelor of Science in Human Biology, Indiana University - Bloomington, IN, 2010

Bachelor of Science in Nursing, Bellarmine University - Louisville, KY, 2012

Master of Science in Nursing, Bellarmine University - Louisville, KY, 2015

A Dissertation Approved on

June 4, 2020

by the following Dissertation Committee:

Dissertation Chair

Barbara Polivka, PhD, RN, FAAN

Becky J. Christian, PhD, RN, FNAP

Whitney Nash, PhD, APRN, FAANP

R. Kevin Mallinson, PhD, RN, AACRN, FAAN 


\section{DEDICATION}

This dissertation presents findings regarding the thoughts and opinions of people who identify as culturally 'Deaf'. In addition, it discusses the representation (and lack of representation) of this population in healthcare research and healthcare settings.

As such, I wish to dedicate this work to members of the Deaf community.

I hope this work contributes to the ever-increasing body of knowledge related to Deaf people and Deaf culture. May it draw attention to the exclusion of Deaf voices in

healthcare literature, be a beacon to researchers regarding the importance of including Deaf voices, encourage change in those who practice within all healthcare fields, and speak truly about the experiences of individuals. 


\section{ACKNOWLEDGEMENT}

This dissertation represents the culmination of five years of study, professional development, and community engagement. However, the success of this project, and the knowledge within, reflects more than my efforts alone. Countless individuals assisted in a plethora of profound ways and deserve credit for their (often voluntary) contributions.

In particular, Dr. Barbara Polivka deserves credit for initiating my interest in obtaining a $\mathrm{PhD}$ in Nursing. In addition, she deserves commendations for maintaining the role as my dissertation chair, despite moving to another state and university. Her encouragement, and dedication to my development, were more than appreciated. I was fortunate to have her as a mentor, and I look forward to becoming her colleague.

Several other individuals deserve credit for contributing to the development and success of this project. These individuals include all of my former instructors, including many from other departments or universities. Some of these individuals and their contributions include Dr. Kevin Mallinson for topic identification, Dr. Lynne Hall for topic refinement, Dr. Paul Clark for data collection, Dr. Becky Christian for data analysis, and Dr. Imelda Wright for dissertation preparation.

Finally, several members of the Deaf community (and the affiliated, ASL interpreter community) were instrumental throughout the project. Eric, Jessica, Moira, Randy, Zach, and others (including the participants) were essential in building my understanding of Deaf culture and increasing my involvement. Thank you to everyone. 


\begin{abstract}
TRUST IN PRIMARY CARE PROVIDERS: PERSPECTIVES FROM DEAF PEOPLE
\end{abstract}

\author{
Greir Ander Huck Flynn
}

June, 4, 2020

Trust is an integral part of the patient-provider relationship, responsible for influencing several key aspects of healthcare behaviors and patient perceptions of health outcomes. Though the topic has previously been discussed by scholars, little evidence exists to support the application of our understanding of trust in culturally Deaf populations. As such, this dissertation seeks to lay the necessary groundwork for further investigations on the topic by first understanding the nature of trust in patient-provider relationships from the perspectives of culturally Deaf people.

The dissertation is comprised of five chapters. The first chapter provides an introduction to salient terms and concepts used throughout the dissertation. It also provides a brief history and description of the literature dedicated to both general trust and trust within the context of healthcare relationships. The second chapter identifies a gap in the patient-provider trust literature by presenting a scoping review of the literature from nursing and allied health fields. The findings of this review highlight a lack of literature regarding patient-provider trust in relation to Deaf culture and those who identify as Deaf. The third chapter discusses unforeseen challenges faced by the primary researcher while conducting cross-cultural research in American Sign Language (ASL) 
as a conversationally fluent individual. Issues establishing linguistic equivalence between English and ASL as well as challenges securing and working with licensed ASL interpreters were described.

The fourth chapter examines the perspectives of individuals who are culturally Deaf in regard to patient-provider trust. Using a qualitative descriptive design, findings from a single focus group discussion and one-on-one interview are presented. The fifth, and final chapter, provides a synthesis of the findings from all previous chapters and makes recommendations for future nursing research, practice, education, and policy.

The primary findings of this dissertation were a large degree of congruence between current models and conceptualizations of trust in patient-provider relationship when compared to the conceptualizations of study participants. General attributes such as ability, integrity, and benevolence appear relevant to participants when discussing the nature of trust in patient-provider relationships. However, the weight and importance of each attribute appears unique for members of Deaf communities. In particular, the themes of sameness, power dynamics, professionalism, and culture clash were evident in transcripts. Likewise, communication was a key overarching theme, containing a unique set of subthemes more prominent for individuals who rely on visual forms of communication than those who predominantly rely of oral forms of communication.

Collectively, this dissertation supports claims made by others regarding the lack of Deaf voices in the healthcare literature and fills a small portion of this gap by focusing on the experiences of Deaf people in patient-provider relationships. In addition, this dissertation highlights the need for researchers and healthcare providers to consider the unique needs of Deaf communities and offers a guidance to achieve this goal. 


\section{TABLE OF CONTENTS}

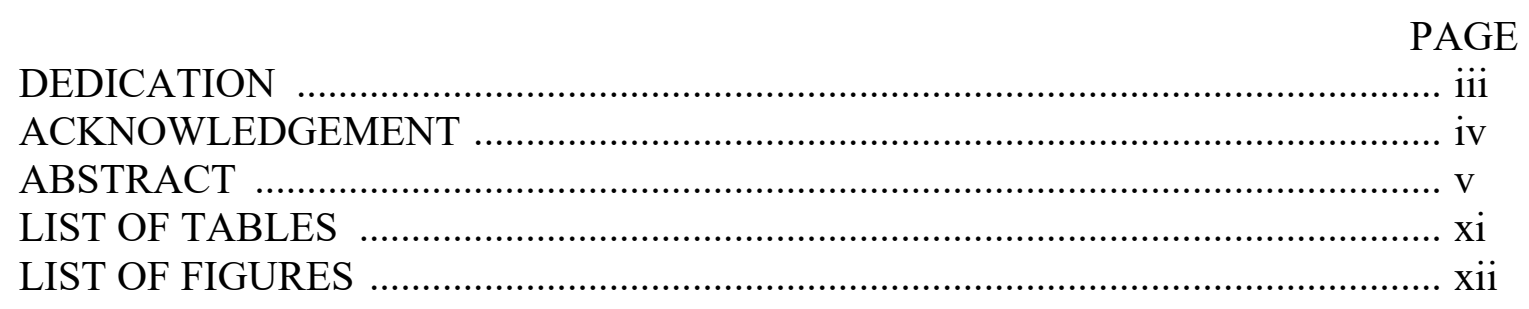

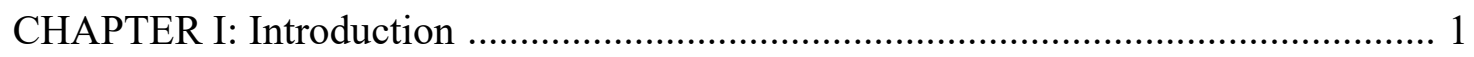

The Importance of Trust in Healthcare Settings ..................................................... 1

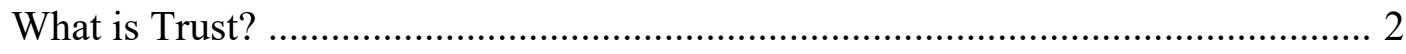

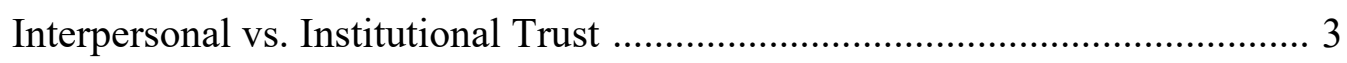

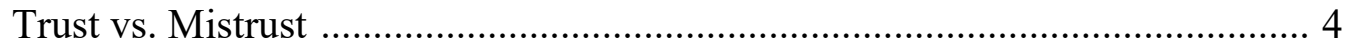

Trust vs. Other Constructs …………………………………………….. 5

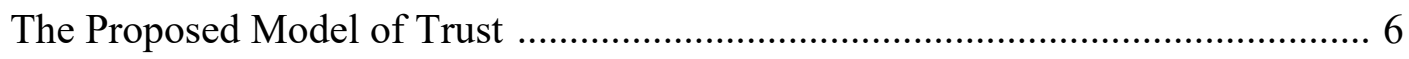

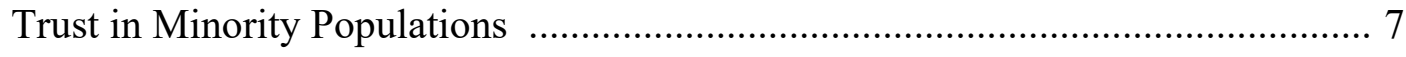

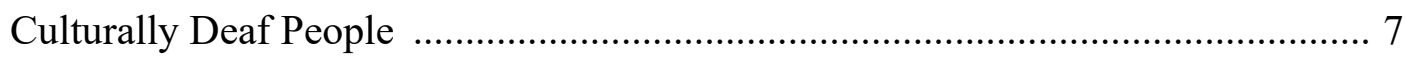

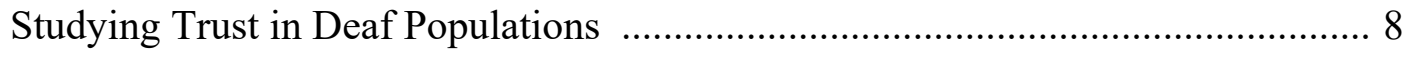

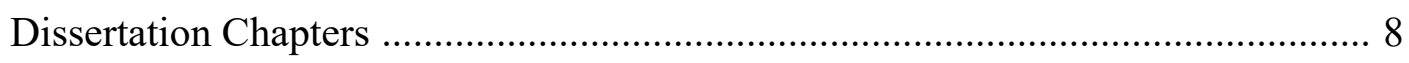

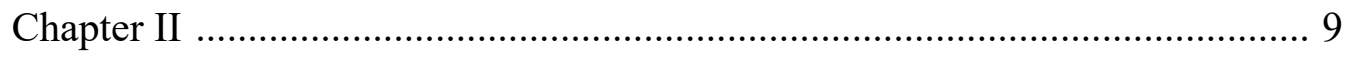

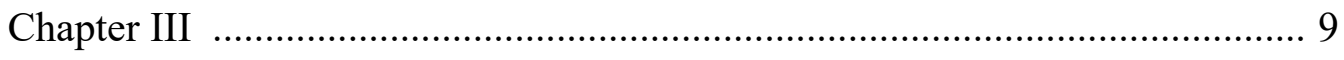

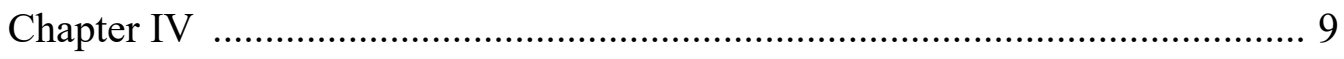

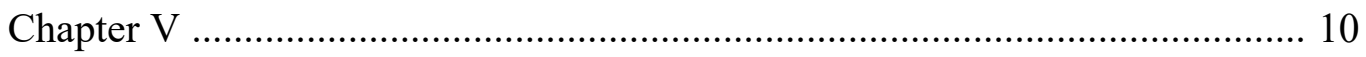

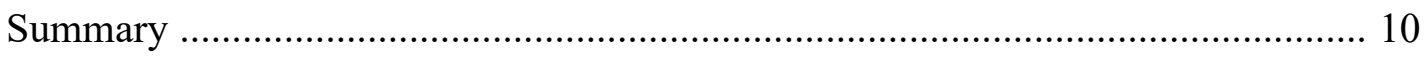


CHAPTER II: Patient-Provider Trust in Deaf Communities .................................... 12

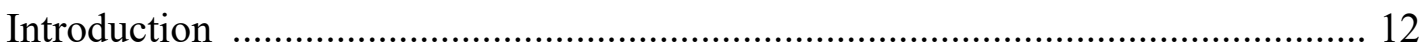

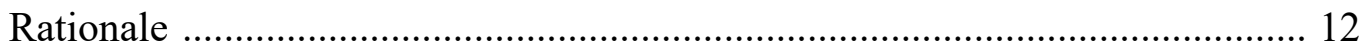

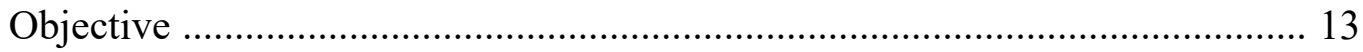

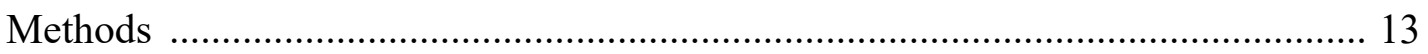

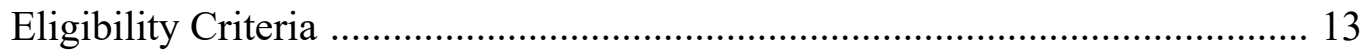

Information Sources [......................................................................... 14

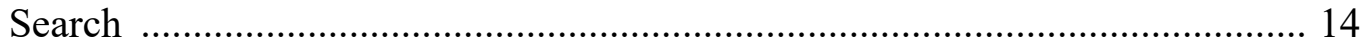

Selection of Source of Evidence .................................................................. 14

Data Charting Process ............................................................................ 15

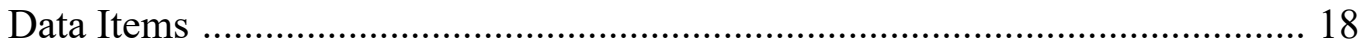

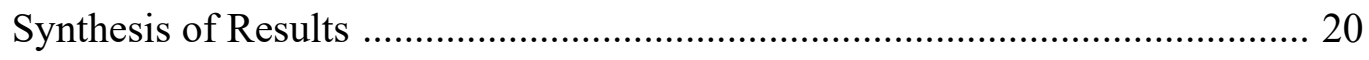

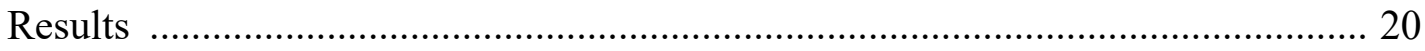

Selection of Sources of Evidence …......................................................... 20

Characteristics of Source of Evidence ...................................................... 21

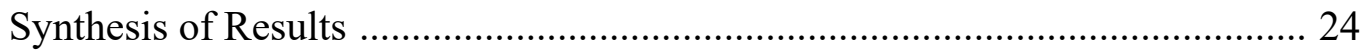

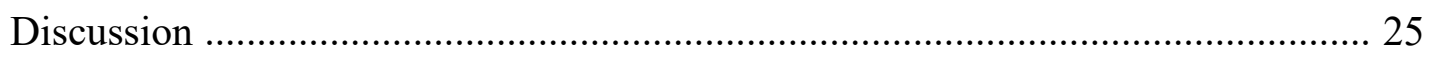

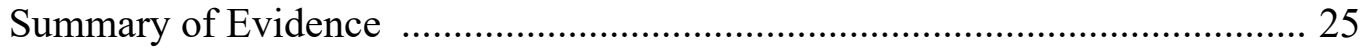

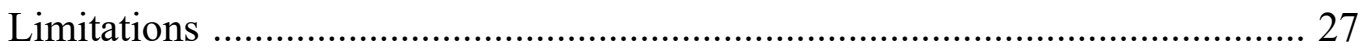

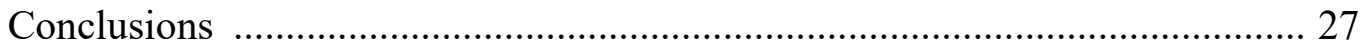

CHAPTER III: Methodological Challenges and Strategies for Cross-Cultural Research

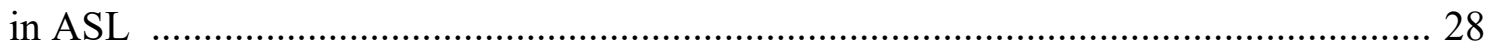

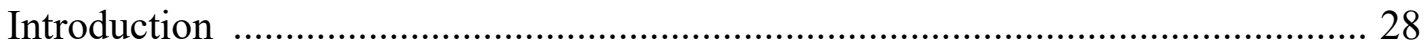




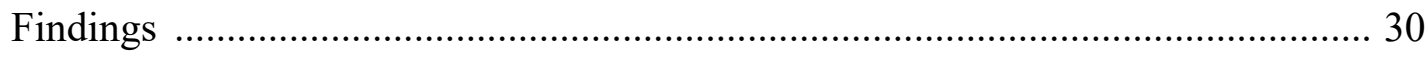

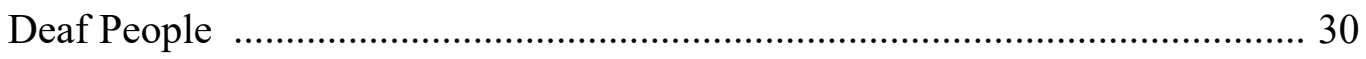

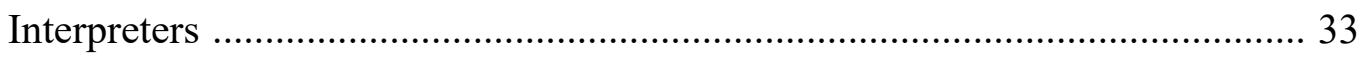

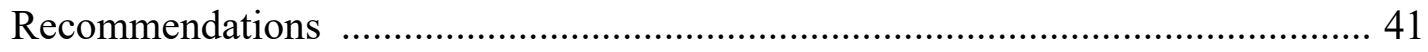

Team-Up with Deaf People ......................................................................... 41

Establish Yourself in the Community .................................................................. 41

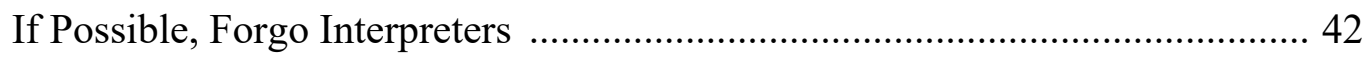

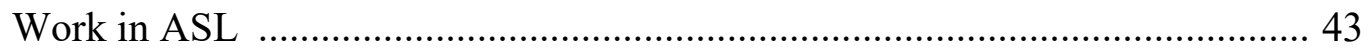

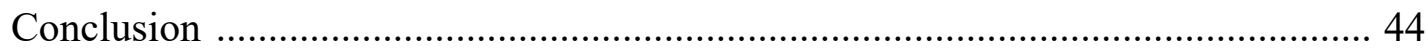

CHAPTER IV: Trust in Primary Care Providers: Perspectives from Deaf Persons ...... 45

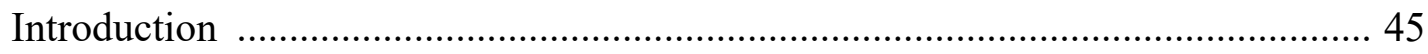

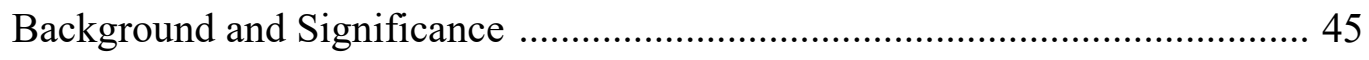

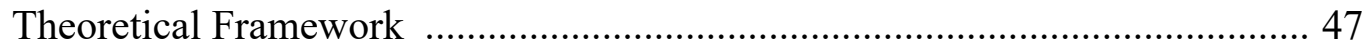

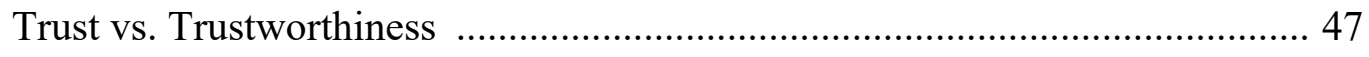

Research Design and Methods ...................................................................... 48

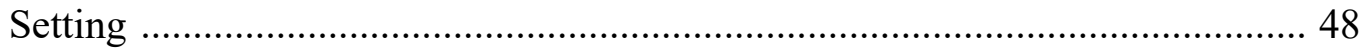

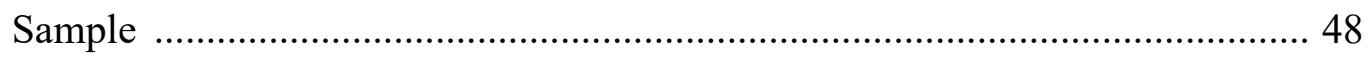

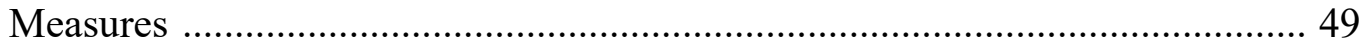

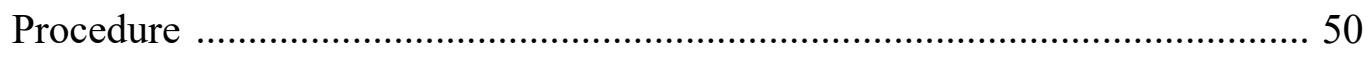

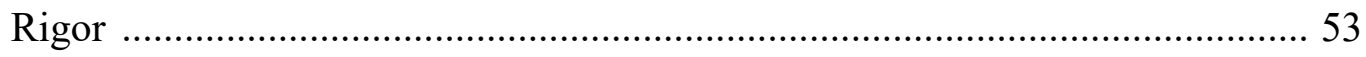

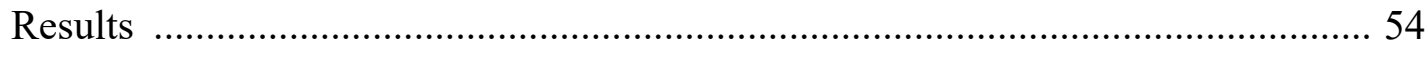

Demographic Characteristics ......................................................................... 54

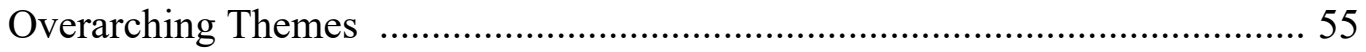




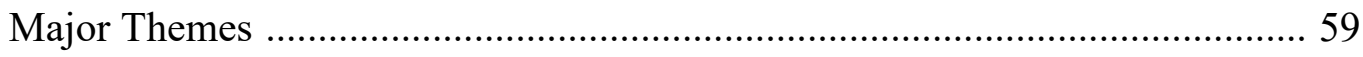

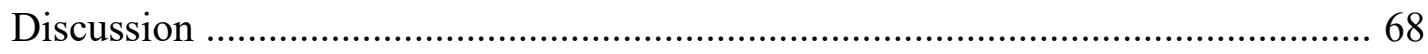

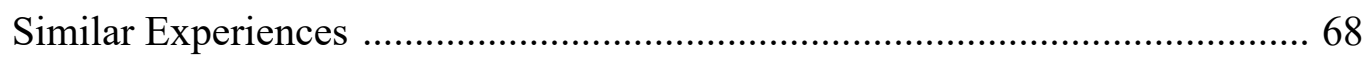

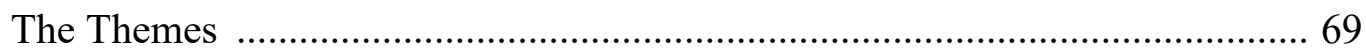

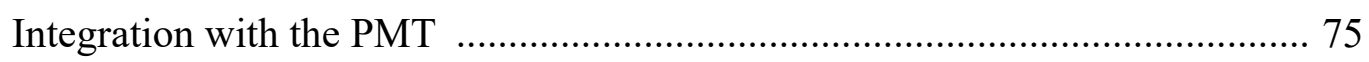

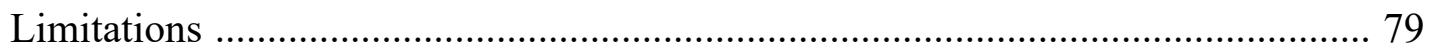

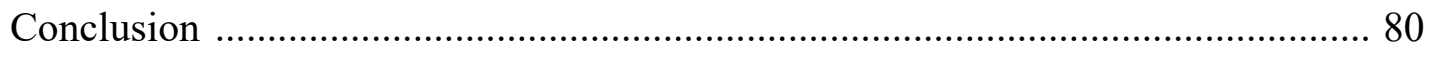

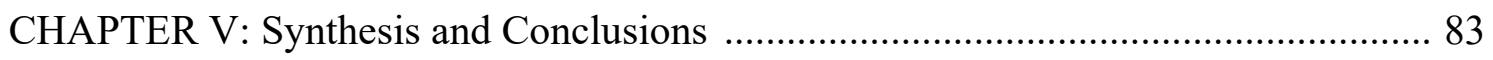

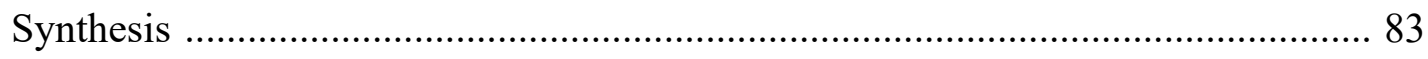

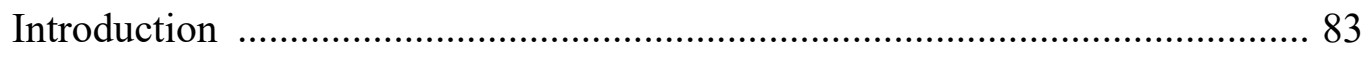

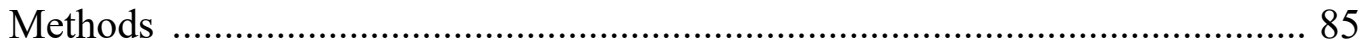

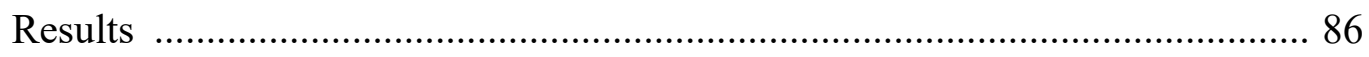

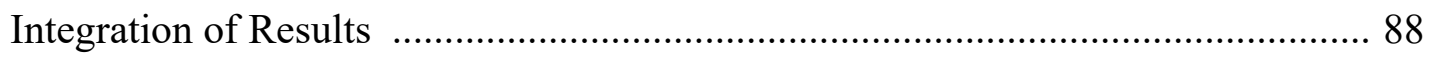

Theoretical Frameworks in Deaf-Focused Research ........................................ 88

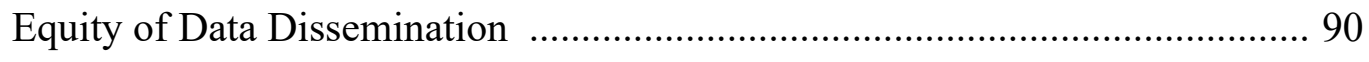

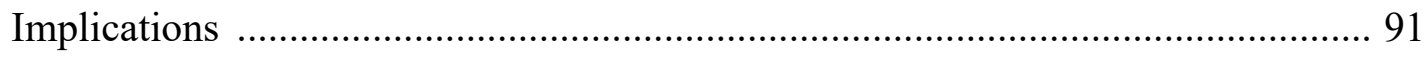

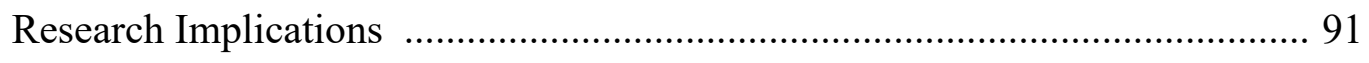

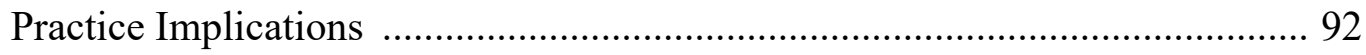

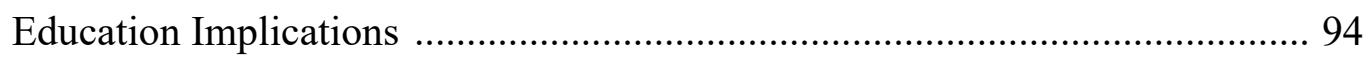

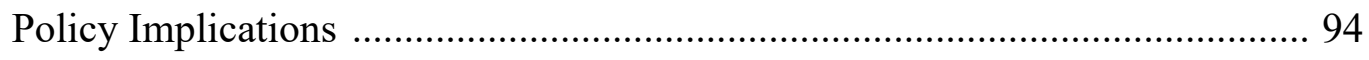

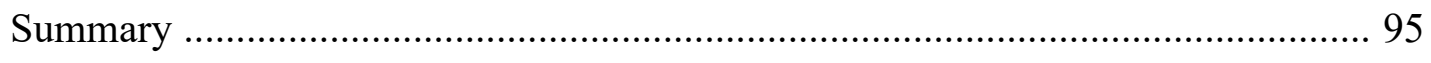

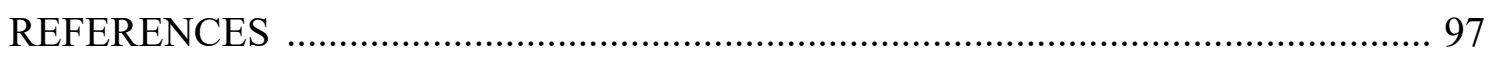

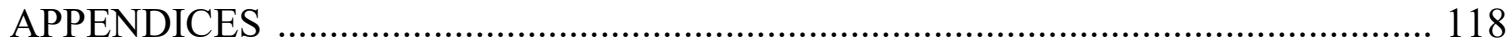

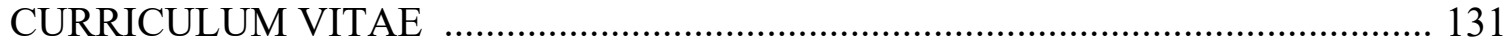




\section{LIST OF TABLES}

TABLES

PAGE

1. Citation Identification, Screening, Review, and Inclusion by Order of Search Terms Used 120

2. Data Charting Form (DCF) Columns by Processing Order of Data 121

3. Articles with Substantive and Cursory Content Related to Trust

4. Frequency of Trust-Related Concepts in Patient-Provider Relationships 125

5. Participant Demographics 128 


\section{LIST OF FIGURES}

TABLES

PAGE

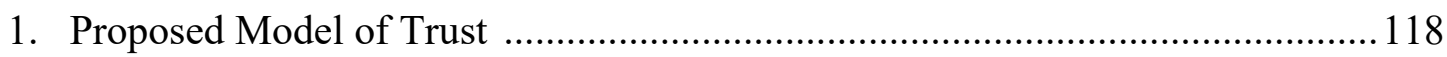

2. Trust Components of the Proposed Model of Trust ........................................ 119

3. Flow Diagram of Study Selection ............................................................ 122

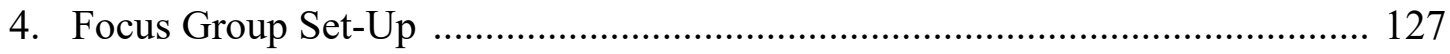

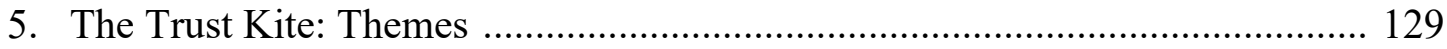

6. The Trust Kite: Trustworthiness ....................................................... 130 


\section{CHAPTER I}

\section{INTRODUCTION}

The purpose of this dissertation is to explore elements of trust within the patientprovider relationship from the perspective of Deaf people. This dissertation uses a qualitative descriptive design to examine factors affecting the perceptions of Deaf participants towards Primary Care Providers (PCPs) with a specific focus on perceptions of a trustworthiness and the propensity to trust others as defined by the Proposed Model of Trust (PMT) by Mayer et al. (1995). Likewise, the concept of trust is generally discussed to verify cross-cultural (and cross-lingual) application of the terms. In total, this dissertation comprises an introduction, three manuscripts, and a conclusive chapter with synthesis of findings. The first manuscript provides a scoping review on trust in patientprovider relationships from the perspective of culturally Deaf people within the nursing and allied health literature. The second manuscript addresses specific challenges, possible solutions, and general recommendations when conducting research with members of Deaf communities. The third manuscript reports the primary findings of this dissertation by using elements of the PMT to explore trust and trustworthiness towards PCPs from the perspectives of Deaf people.

\section{The Importance of Trust in Healthcare Settings}

perceived health gains (Caterinicchio, 1979; Hall et al., 2001). In addition, a combination of factors influenced by trust are theorized to impact several aspects of healthcare including delivery costs, overall use, and efficacy of the healthcare system as a whole (Thom et al., 2004). Fortunately, aspects of trust in healthcare professionals remains high with $85 \%$ of the general US public indicating nurses are both honest and ethical in their 
practice, followed by engineers (66\%), physicians (65\%), pharmacists (64\%), and dentists (61\%) (Jones \& Saad, 2019). Over the past two decades, healthcare professions have each seen a steady increase in trust (Jones \& Saad, 2019) amid growing concerns over increased levels of mistrust and decreasing levels of trust reported by others (Daniel J. Edelman Holdings Inc., 2019; Blendon et al., 2014; Mechanic, 1996; Platt et al., 2018). Factors influencing this discrepancy include differences in the conceptualization of trust, the instruments used to measure trust, and the demographics of the populations surveyed. However, additional reported rationale includes changing clinical recommendations due to exponential increases in medical advances (Lynch et al., 2019), and contentious media coverage of physician advocacy in political arenas (Blendon et al., 2014; Mechanic, 1996). Regardless of the cause or existence of any discrepancy, trust is universally acknowledged as an important concept in healthcare and worthy of further consideration.

\section{What is Trust?}

Trust may be universally acknowledged and experienced, but much debate exists regarding its true (or empirical) nature. In its broadest sense, trust is the belief an individual holds about the probability of a future reality, and it became a concept of interest in the late 1950s to early 1960s with the work of Deutsch, an American social psychologist interested in conflict resolution. At the time, Deutsch $(1958 ; 1960)$ theorized that trust contained two dimensions: perceptions of outcomes and anxiety. These dimensions of trust were later adopted by healthcare researchers (Caterinicchio, 1979) amid a surge of philosophical debates regarding the dimensionality of trust (Baier, 1986; Bok, 1978; Luhmann, 1979). Though these original dimensions of trust hold less 
prevalence among the patient-provider trust literature, new scientific inquiries emerged from these debates, reshaping the trust literature in profound ways.

Bok (1978) was among the first to identify different types of trust, providing three categories: fairness, self-interest, and results. Though still removed from modern conceptualizations of trust in healthcare arenas, Bok challenged theorists to investigate the dimensionality of trust and the transferability of trust in different settings. Luhmann (1979) mirrored Bok's sentiments; however, Luhmann (1979) suggested two types of trust: interpersonal and systems. Within healthcare, Luhmann's work can be seen in the debate over the relevance of interpersonal or institutional trust in patient-provider relationships (Hall et al., 2001). Additional complexities, such as the relationship between trust, mistrust and conflated terms, were later enhanced by the work of Baier.

\section{Interpersonal vs. Institutional Trust}

Many scholars believe interpersonal trust, or the trust an individual has towards another, holds sway over patient-provider relationships because at their core, patientprovider relationships require some form of interaction between two specific people: the person providing the service and the person receiving the service. Therefore, several models of trust and instruments used to measure trust within patient-provider relationships target levels of interpersonal trust (e.g., Anderson and Dedrick (1990), Kao et al. (1998), and Stepanikova et al. (2006)). In contrast, many scholars believe institutional trust, or the trust an individual has towards an organization, or a collection of relatively unknown individuals, is the best way of understanding trust within patientprovider relationships due to the ever-increasing ubiquity of technology and the changing healthcare system (Hall et al., 2001). These scholars see the interaction between patients 
and providers as being moderated by circumstantial factors including the need for

patients to see affiliated providers instead of their own PCP (Leisen \& Hyman, 2001) and the need to trust a network of unidentified individuals, like technologists and technicians, who work behind the scenes to support PCPs (Rose et al., 2004). Moreover, some scholars see patient-provider trust as being mediated by circumstantial factors like technology (van Velsen et al., 2017), data sharing (Platt et al., 2018), and interactions through public forums (Buhr \& Blendon, 2011).

In some cases, scholars incorporate both interpersonal trust and institutional trust into their analysis by acknowledging the separation between the two (Russell, 2005). Others unite the two in a holistic approach to the patient experience (Lynch et al., 2019), and still others combine these techniques by separating and uniting the two forms of trust by examining their mediating effects on each other (Gratz, 2018).

\section{Trust vs. Mistrust}

Within the healthcare literature, the relationship between trust and mistrust (or trust and distrust) is conceptualized in two conflicting, cardinal configurations. The first, and possibly most prolific, asserts that trust and mistrust exist on a continuum. On one side of the continuum "trust" is placed and on the other side of the continuum mistrust (or distrust) is placed. Alternatively, some scholars substitute mistrust with an utter lack of trust (i.e., no trust), believing the absence of trust - even when combined with distrust does not infer the existence of an opposing concept (Schoorman et al., 2007). These individuals believe all aspects of trust, and its absence, exist on one continuum.

The second prevalent configuration of trust and mistrust avers that trust and mistrust are two separate constructs best represented by two perpendicular axes. One axis 
contains a trust/lack-of-trust continuum, the other contains a mistrust/lack-of-mistrust continuum. With this configuration, individuals can simultaneously hold high levels of trust and mistrust, low levels of trust and mistrust, or any combination in between (Rose et al., 2004; Wang et al., 2009). The difference between these two configurations of trust influence several facets of scholarship including which model or theoretical frameworks to base scientific inquiry, which instruments to use when conducting trust research, and how to interpret data. Among those working with minority populations, a proclivity for the biaxial configuration exists. The majority of this research unilaterally focusing on the mistrust axis. However, some scholars, like Stepanikova et al. (2006) and Wang et al. (2009), acknowledge or incorporate both axes in their research.

\section{Trust vs. Other Constructs}

Like many abstract concepts, trust is difficult to distinguish from similar, but separate, constructs. For example, reliance is closely aligned with trust. While both hinge on future outcomes, trust is given in situations where more than one option is presented and reliance is coerced when there is only one possible source of assistance (Baier, 1986). Communication is also different from trust. Though communication is an integral part of trust (Zhao et al., 2016), communication is the means by which meaning is conveyed and trust is a prediction about the future based on the interpretation of the information conveyed. Communication is a salient domain in $93 \%$ of instruments used to measure trust in the health literature (Ozawa \& Sripad, 2013), but what is directly or indirectly communicated (e.g., integrity or knowledge) - not the communication itself is the dimension of trust (Mechanic \& Meyer, 2000). Finally, several alternative concepts are used as proxies for trust in both formal and colloquial discussions. These proxies are 
copious, and include satisfaction, integrity, and confidence. Many times, these substitutions are inaccurate (Luhmann, 1979), but often they are one of several dimensions of trust (Schoorman et al., 2007).

\section{The Proposed Model of Trust}

This dissertation conceptualizes trust in accordance with the PMT (Mayer et al., 1995). This model was originally created as an organizational theory to explain the feedback loop associated with an individual's level of trust towards, and subsequent behavior with, an organization (Mayer et al., 1995). It identifies trust as a uniaxial construct, which both differentiates and incorporates several conflated concepts, all while assessing either institutional and interpersonal trust, depending on the 'organization' identified (Schoorman et al., 2007). For this dissertation, the 'organization' in question is PCPs generally, with many participants recalling specific interactions with established providers.

The PMT has four major components, arranged in a linear fashion, with each component sequentially influencing the next (see Appendix A). This dissertation focuses on the first two components (i.e., 'factors of perceived trustworthiness' and trust), and the moderating component, 'propensity to trust' (see Appendix B). Working backwards, trust is defined as the willingness to be vulnerable (Mayer et al., 1995), which requires an individual to consciously consider the probability of a desired outcome based on three attributes: the trustee's ability, integrity, and benevolence. Ability is situational, asserting that having skills for one task does not ensure success of other tasks. Integrity incorporates both the acceptance of, and adherence to, the same set of principles. Benevolence implies a degree of attachment and desire to do 'good' things for the 
individual. All three attributes are moderated by the trustor's propensity to trust others. Incorporating the trustor's propensity to trust others as a moderating variable accommodates individual and cultural trends that influence an individual's ability to trust others.

\section{Trust in Minority Populations}

General metrics for trust in healthcare providers might be high (Jones \& Saad, 2019), but several researchers have found mistrust (or distrust) of providers high among several American subcultures. In particular, minority populations like Blacks/African Americans (Boulware et al., 2003) and Hispanics (Altice et al., 2001), as well as other marginalized populations like women and individuals with lower incomes (Blendon et al., 2014) report higher levels of mistrust than affluent white males. However, a general search of the healthcare literature did not reveal any studies reporting levels of trust or mistrust among people who are culturally Deaf, despite a traumatic and oppressive history with healthcare providers and members of healthcare-affiliated professions (Kaplan, 1996; Lane, 1999). Several studies do reference a general degree of mistrust among culturally Deaf individuals towards the hearing world (Steinberg et al., 2006), and healthcare providers (Steinberg et al., 1998). However, these publications predominantly focus on sharing the experiences of Deaf people in healthcare settings rather than addressing the concept of trust (or mistrust) as it is addressed in other publications pertaining to individuals from other populations.

\section{Culturally Deaf People}

People who identify as 'Deaf' (spelled with a capital 'D') exist as a unique subculture in American society. Like other subcultures Deaf people have their own 
language, art, belief systems, and cultural norms (Moore \& Levitan, 2011), that can sometimes be at odds with hearing and healthcare cultures (Meador \& Zazove, 2005). They also have a history of being misunderstood and oppressed by hearing majority cultures (Lane, 1999), with healthcare and allied providers frequently assuming the role of 'villain' by promoting cochlear implants and continuing to display a lack of cultural competence (Kaplan, 1996; Lapinksi, 2015; Nagakura, 2015). However, a paucity of information regarding trust between Deaf patients and hearing providers exists in the healthcare and allied health literature, suggesting the need for further exploration.

\section{Studying Trust in Deaf Populations}

Understanding the concept of trust from the perspective of Deaf people, especially within the patient-provider relationship, will provide greater understanding of the innerworkings of patient-provider relationships. However, prior to investigating this relationship it is prudent to investigate the current state of the science regarding patientprovider relationships from the perspective of Deaf people as the knowledge gained from this activity can be used to improve the future study designs in the area of interest.

\section{Dissertation Chapters}

This dissertation is comprised of five chapters. This chapter is followed by a manuscript for a scoping review of healthcare-related trust literature in Deaf populations, a manuscript recalling challenges met conducting research in American Sign Language (ASL) with licensed ASL interpreters, and a manuscript presenting data from a focus group and interview with Deaf people on PCPs and their level of trustworthiness. The final chapter provides a synthesis of these manuscripts with commentary on future implications of this work. 


\section{Chapter II}

Chapter II of this dissertation presents a scoping review of the nursing literature on trust and deafness, with a focus on trust within patient-provider relationships as perceived by people who identify as culturally Deaf. To collect these data, the Preferred Reporting Items for Systematic Reviews and Meta-Analyses extension for Scoping Reviews (PRISMA-ScR) guidelines were followed: a predetermined set of criteria were established, citations from the Cumulative Index to Nursing and Allied Health Literature (CINAHL) were compiled, and a detailed report of identified publications is presented. These data are synthesized, highlighting the gaps in available information dedicated to understanding patient-provider trust within Deaf communities.

\section{Chapter III}

Chapter III presents challenges to conducting cross-cultural research with members of Deaf communities from the perspective of a conversationally ASL-fluent researcher with seven years of community involvement. This chapter focuses on communication challenges including the use of ASL and interpreters when recruiting and working with Deaf participants. In addition, this chapter provides solutions to presented problems and recommendations for other researchers interested in conducting research with this population. Examples include the importance of incorporating members of the Deaf community into the research team and establishing linguistic equivalence throughout the research process.

\section{Chapter IV}

Chapter IV of this dissertation presents the findings of a qualitative study focused on trust in PCPs, as perceived by people who identify as culturally Deaf. The purpose of 
this chapter is to present the common themes expressed by participants and their level of congruence with established conceptualizations of trust. Identified themes include (a) sameness, (b) power dynamics, (c) professionalism, and (d) culture clash, with communication as an overarching theme. Additional analysis of these themes in conjunction with the theoretical framework (i.e., the PMT) is also presented.

In general, members of the Deaf community perceived trust in ways consistent with members of other communities, though the specifics of their experiences and perceptions contained similarities and differences within the group and across groups. The three attributes of perceived trustworthiness outlined by the PMT (i.e., ability, integrity, and benevolence) were consistently expressed, with the attributes of ability and integrity holding more weight than benevolence. In addition, several participants expressed an underlying desire to trust others, but an inability to extend this propensity to members of hearing cultures.

\section{Chapter V}

Chapter V provides a synthesis of the findings from Chapters II, III, and IV. In addition, an argument is made for further inclusion of Deaf people in healthcare research as well as a greater emphasis on incorporating healthcare concepts into the design and analysis of data collected from Deaf participants.

\section{Summary}

In summation, trust within patient-provider relationships constitutes a developed body of research from which Deaf people and Deaf patients appear to be excluded. This dissertation seeks to identify the extent to which research on patient-provider trust has incorporated the experiences of Deaf people and what investigate any differences that 
might stem from cultural differences. Finally, recommendations for future scholars will be made. 


\section{CHAPTER II}

\section{PATIENT-PROVIDER TRUST IN DEAF COMMUNITIES:}

\section{A SCOPING REVIEW OF NURSING LITERATURE}

\section{Introduction}

\section{Rationale}

Trust is an integral part of the patient-provider relationship influencing several aspects of care. Use of preventative care services (Russell, 2005; van Velsen, 2017), adherence to treatment regimens (Caterinicchio, 1979; Hall et al., 2002; Penman et al., 1984), and continuity of care (Thom, 1999; van Velsen, 2017) have been positively correlated with a patient's levels of trust in their provider. To date, valid and reliable instruments exist to measure trust between patients and providers (Ozawa \& Sripad, 2013), and these instruments have been used to study levels of patient-provider trust in a variety of cultural groups (Müller et al., 2014). However, a meta-analysis on the use of patient-provider trust instruments revealed a dearth of publications using trust instruments with culturally Deaf populations (Müller et al., 2014). Furthermore, an overview of literature in the fields of healthcare, education, and business produced few citations addressing the concept of trust in conjunction with Deaf populations. However, several tangential publications were identified. The lack of definitive evidence revealed in three systematic reviews regarding trust in healthcare providers (Müller et al., 2014; Ozawa \& Sripad, 2013; Røtveit et al., 2015), and the aforementioned independent review 
suggests the need for deeper exploration into the current discussion of patient-provider trust as it pertains to people who identify as culturally Deaf.

\section{Objective}

The purpose of this scoping review is to identify the use of the term 'trust' (and its derivatives) within the nursing and allied health literature. Borrowing an organizational theory about trust (Mayer et al., 1995) and incorporating other healthcare conceptualizations of trust (Ozawa \& Sripad, 2013), this scoping review seeks to present a holistic view of trust-related discussions surrounding Deaf patients and their providers by identifying the extent to which tangential concepts to trust are discussed in the nursing and allied health literature. The results indicate whether a systematic review of the literature pertaining to trust within patient-provider relationships is warranted for Deaf populations and what key terminology should be incorporated in such a review.

\section{Methods}

\section{Eligibility Criteria}

The content of this review was only limited by the search terms, database, and aggregator used. As such, no filters were placed on the type of material (e.g., journal article), quality of the material (e.g., peer-reviewed), extent to which the content was accessible (e.g., full-text availability), language of publication (e.g., English), or location of search terms within the material's citation (e.g., title). In addition, no filter was placed on the date of publication (e.g., last ten years); however, the database used only referenced publications from 1961 onward. These search parameters were chosen after a filtered pilot search using key terminology revealed few favorable results. Eliminating 
traditional filters permitted the greatest amount of referenceable data and the widest capture of the topic in the literature (Arksey \& O’Malley, 2005).

\section{Information Sources}

The University of Louisville's electronic 'World Catalog' was used to perform all searches in the Cumulative Index to Nursing and Allied Health Literature (CINAHL) database. Preliminary searchers were completed between May and June of 2018 with additional searches in February of 2020. Additional citations discovered in reference lists of CINAHL-identified articles were retrieved through a variety of additional sources including Google Scholar and PubMed.

\section{Search}

Several rounds of searches were conducted for this review, each incorporating additional synonyms for trust. This process resulted in crossing the term 'deaf' with fifteen additional search terms. A full list of search terms and the resulting number of citations found in CINAHL from each pairing is provided in Appendix C. In several instances, truncated search terms such as 'empath*' were used instead of performing multiple searches with more specific terminology (e.g., empathy and empathetic). In addition, reference lists from reviewed articles identified 13 additional citations.

\section{Selection of Sources of Evidence}

The CINAHL database was chosen because it is the primary source of nursing literature, indexing more than 5,500 journals in nursing and related fields (EBSCO Information Services, 2020). The University of Louisville's 'World Catalog' was chosen to collect citations as it permitted access to full-text versions of citations through a university account. Sources used to retrieve additional citations were chosen by their 
ability to provide a full-text version of the desired citation. No filters were used in conjunction with any citation source.

\section{Data Charting Process}

\section{Creating the Data Charting Form}

Citations were originally extracted from the World Catalog in bulk using the embedded 'export to citation manager' option. A limit on the number of exportable citations required the results of some search term combinations to be extracted in batches, but all available citation fields were transferred including abstracts and hyperlinks. Fulltext articles pertaining to each citation were not initially retrieved.

These citations were then transferred to the citation manager EndNote where citations were sorted into folders ('groups') based on the search terms used to identify them. After all citations were added to EndNote, duplicates within groups and across groups were identified using the 'find duplicate' feature and searching for citations with identical authors, years of publication, and article titles. Two new groups were created, one containing a single copy of every retrieved citation and one containing any duplicate citations.

After the duplicates were removed, a 'data charting form' (DCF) was created per the recommendations of Arksey and O'Malley (2005) in which a single copy of each citation was extracted from EndNote and imported into Excel. A total of 1566 citations were transferred in this manner. Transferred citation fields included the author, year of publication, title, abstract, and EndNote reference number. During the data charting processing, the author and publication year were used to identify citations within the DCF 
and the EndNote reference number was used to quickly identify the citations between the DCF in Excel and the repository of citations in EndNote.

Additional fields were then generated as columns on the DCF to record the stage of processing (i.e., review of title, abstract, or article), target population of the citation (e.g., health experts), conceptual topic of the citation (e.g., educational needs), and relevance to the investigation (e.g., trust). Later, additional fields were generated to distinguish between articles discussing trust, mistrust, and a variety of trust-related concepts. A full list of generated fields can be found in Appendix D.

\section{Using the Data Charting Form}

Citations were retrieved, compiled, and processed by a single investigator over the course of several months. Using the DCF, the investigator first read the title of each citation, then the abstract, and finally the full-text version of the citation if each of the previous (i.e., abstract or title) indicated the need for further investigation. Initially the investigator reviewed the citation material provided by the World Catalog in search of articles addressing trust or trustworthiness in patient-provider relationships from the perspectives of culturally Deaf patients. Later, full-text versions of potentially relevant citations were retrieved and reviewed to better differentiate how (and whether) trust was discussed in each citation.

To minimize false negatives during citation identification, the investigator assumed all citations addressed the perspectives of Deaf patients towards healthcare providers until this assumption was determined to be inaccurate. For example, the article titled Association between intention for physical activity practice, social support and physical activity (de Paiva et al., 2016) was filed among the citations in need of further 
investigation because its title and abstract did not rule out a discussion about trust in healthcare providers from the perspectives of patients who are Deaf. However, after reviewing the full-text version of the citation, the article by de Paiva et al. (2016) was excluded from the scoping review because it did not address trust, nor did it address people who are Deaf.

When recording in the DCF, citations at each stage of the investigation (i.e., title, abstract, and full-text article) were first removed from further investigation based on the target population of the citation, and then by the conceptual topic of the citation. For example, an article by Vermeulen et al. (2007) titled Reading comprehension of deaf children with cochlear implants was recorded and 'removed' from further investigation due to the target population (i.e., people with cochlear implants). Though the conceptual topic of this citation (i.e., education) also excluded the citation from further review, the conceptual topic of the citation was not recorded. Likewise, this citation's title suggests multiple target populations including non-healthcare-related professionals, but only one target population was recorded in the DCF.

Data for citations that underwent full-text review and addressed trust or trustrelated concepts were further delineated into a number of categories. First, articles referencing trust were filed as providing substantive content on trust (i.e., substantive trust), cursory content on trust (i.e., cursory trust), or both. Though many articles only contained one reference to trust, articles providing both substantive and cursory content on trust were double filed. Likewise, articles referring to trust-related concepts were filed under substantive concepts, cursory concepts, or both. In these situations, which trustrelated concepts the article contained were recorded. Finally, due to this data recording 
strategy, it was possible for a single article to be filed under all substantive and cursory categories of all types (i.e., substantive and cursory trust, as well as substantive and cursory trust-related concepts).

\section{Data Items}

\section{Target Population of Citation}

Citations referencing people with cochlear implants, blindness, and general disabilities were filed under the corresponding exclusion heading of the same name regardless of deafness. Though individuals with cochlear implants, blindness, and disabilities may identify as culturally Deaf, these individuals interact with the world and are perceived by the world - in alternative ways. Therefore, their perspectives were excluded from this scoping review. Citations focused on the opinions of individuals who were affiliated with, but were not, culturally Deaf were filed as either a) patients and family members, b) healthcare and healthcare-related professionals, c) non-healthcarerelated professionals, or d) other hearing people.

Healthcare professionals included providers at any level including all PCPs, pharmacists, nurses, and nursing aides. Healthcare-related professions included audiologists, social workers, and speech pathologists. Non-healthcare-related professions included teachers, unidentified researchers, economists, and theorists. Specific fields were created to identify citations pertaining to American Sign Language (ASL) interpreters and organizational trusts. Other hearing people included anyone not previously categorized. Examples include citations focused on the experiences of hearing people and performance comparisons made between deaf and hearing people. Any 
citations identified as focusing on the opinions or experiences of people with Deaf identities were differentiated further by their topic.

\section{Conceptual Topic of the Citation}

Eight conceptual topics were identified as beyond the topics of interest (i.e., trust and trust-related concepts). These eight topics included epistemology, experiences, education, social interactions, technology, health outcomes, knowledge, and cognition. Epistemology focused on the knowledge and beliefs Deaf people have about their own history or culture. Experiences focused on non-healthcare-related experiences. Education included all elements of learning, reading, and educational theory. Citations filed under social interactions focused on professional outcomes, personal choices, and other topics involving direct interactions with other (typically hearing) people. Technology included opinions about cochlear implants and other assistive devices as well as the perspectives of Deaf people toward healthcare procedures. Health outcomes focused on genetic issues, physical development, health maintenance, and access to care. Knowledge included beliefs, behaviors, and access to knowledge, including healthcare information about other people or other things. Finally, cognition focused on psychological development, including language, personality, and general knowledge acquisition.

\section{Trust-Related Concepts}

The focus of this scoping review concerned the concept of trust and its derivatives (e.g., mistrust). However, due to the underrepresentation of trust and mistrust in the healthcare literature surrounding Deaf populations, additional concepts were investigated. Citations including meaningful discussions about trust or mistrust were identified as 'substantive trust' articles, while articles providing perfunctory descriptions of trust or 
superficial acknowledgement of trust without contextual support were identified as 'cursory trust' articles. Likewise, articles providing substantive and cursory commentary on a series of trust-related concepts were respectively named, before further identifying the trust-related concepts of each.

Delineation into a trust-related category typically required use of the relevant term (e.g., use of the word 'ability' to be filed under 'Ability') or a term's derivative (e.g., use of the word 'unable' to be filed under 'Ability'). However, use of some terms permitted an article to be filed under a similar trust-related concept. For example, several antonyms are included in the concept 'Comfort', including shy, insecure, and fear. A complete list of trust-related concepts can be found in Appendix D.

\section{Synthesis of Results}

Data were summarized by first compiling the list of articles included in the fulltext review. For each article, previously charted information (e.g., authors) and compiled data (e.g., examples of substantive trust) were extracted from the DCF and inserted into a new tab within Microsoft Excel. From here, trends in the data were identified by comparing articles within each of the trust and trust-related concept categories.

\section{Results}

\section{Selection of Sources of Evidence}

Of the 1,925 citations initially retrieved, 106 articles were deemed eligible for review. The remaining 1,460 were excluded from review during the screening process for various reasons. The most common reason for exclusion was a lack of target population inclusion $(n=807)$. The majority of these citations focused on people with cochlear implants $(n=461)$ while other citations addressed the perspectives of hearing people 
towards people who are Deaf. The remaining citations $(n=653)$ were excluded for not incorporating trust or a trust-related concept in the article. Examples include citations focused on the visual perceptions, educational strategies, and knowledge sets of Deaf people. In addition, 13 eligible citations were not reviewed. Five of these citations were not written in English, despite their English titles and abstracts. The remaining eight were not able to be retrieved. A flow diagram related to evidence selection can be found in Appendix E.

In addition, the number of citations identified using the original search term (i.e., 'trust') and its derivatives (i.e., 'trust*', 'distrust', and 'mistrust') comprised less than $2 \%$ $(n=25)$ of the total number of citations identified during the identification phase of this review. Of the additional 11 search terms used, 'empath*', 'suspic*', 'believ*', 'rapport', 'opinions', and 'loyal' provided fewer than 40 citations each and represented $5.5 \%$ of the identified citations $(n=87)$. The search term 'perception' identified $47.5 \%$ of the citations $(n=746)$, with 'attitude' $(n=542)$ representing an additional $34.5 \%$. A list of search terms used, and the number of citations identified by cross-referencing each search term with the term 'deaf', can be found in Appendix C.

\section{Characteristics of Sources of Evidence}

A total of 93 full-text articles were reviewed for this scoping review. Data were extracted from each and then used to categorize each citation accordingly. Articles that yielded no identification or discussion about trust or trust-related concepts - as they pertained to members of Deaf communities - were removed $(n=42)$. The remaining articles $(n=51)$ were categorized according to the degree to which each identified and discussed trust and trust-related concepts within the patient-provider relationship from the 
perspectives of Deaf people. These categories were (a) substantive trust articles, (b) cursory trust articles, (c) substantive trust-related concepts, and (d) cursory trust-related concepts. Though each category exists independent of the others, several articles were filed in more than one category because they included a combination of substantive and cursory examples of trust and trust-related concepts (see Appendix F).

In addition, citations containing trust-related concepts were further delineated by the type of trust-related concept identified (e.g., communication versus confidentiality). A complete list of citations and their respective trust-related concepts can be found in Appendix G.

The demarcation between substantive and cursory forms of trust and trust-related concepts was the extent to which examples of the terms were provided in the text and discussed in context with the experiences of Deaf people as a whole. For example, an article stating the following was filed under substantive trust:

thereby allowing members of the community to 'hear about your experience, qualifications' and 'trust what [they] see for [them]selves.' This emphasis on overcoming mistrust is not unique to recruiting Deaf research participants, is a common thread that weaves through culturally-sensitive empirical work with any marginalized or oppressed group [citation] (Anderson et al., 2017a, p. 125)

In contrast, the following citation references a derivative of trust, trustworthiness, but does not discuss the term in context with other conceptualizations of trust or identify what attributes contribute the trustworthy identity in the following passage: "the uncertainty of their knowledge created an eager and energetic desire to understand and verify knowledge from sources they might identify as trustworthy" (Ferguson-Coleman et al., 2014, p. 677). Therefore, this passage would permit the article to be filed as cursory trust. 
Similar differences are seen with the use of trust-related concepts like this quotation from Horne and Pennington, (2010), "Assessment and diagnosis relies on the skills of communication and interpretation" (p. 357). When compared to the following passed by Iezzoni et al. (2004):

Inadequate communication can embarrass patients. "You write back and forth," recalled John, "and the doctor wrote 'C-O-K-E.' I said, 'Yes, a lot.' Suddenly, there were 3 people trailing me to the bathroom for a urine test. I thought they were trying to keep me from running away! I didn't understand where that was coming from. I thought he meant do I drink Coca-Cola. Why didn't he write the whole word 'cocaine'? It's not just 'coke.' (p. 359)

The first example does little more than acknowledge the existence of communication while the second example makes a claim about communication and supports this claim with an example. As such, the Horne and Pennington (2010) article was filed as a cursory trust-related article while the Iezzoni et al. (2004) article was filed as a substantive trustrelated article.

The majority of trust and trust-related concepts identified in articles easily aligned with their respective substantive and cursory categories. However, some examples proved more difficult to determine the extent to which the term needed to apply to the healthcare provider. For example, the passage by Anderson et al. (2017a) specifically discusses trust in healthcare research and in clinical healthcare researchers. Likewise, several articles discussed confidentiality in regard to private health information but directed the concern towards licensed interpreters. In both cases, these examples were included in this review because their concepts (as discussed) applied in the healthcare setting, and the concerns expressed could easily apply to the unnamed healthcare provider in these scenarios. 


\section{Synthesis of Results}

Of the 93 full-text articles reviewed, 51 discussed trust or a trust-related concept (see Appendix F). Of these, 11 articles discussed trust in a substantive manner, but many fell short of providing a robust discussion about trust from the perspective of Deaf people, and in some cases, applying the term substantive may have been an overreach. A few of these articles like Anderson et al. (2017a) provided a rich discussion about the nature of trust from the perspective of its participants, but other articles did little more than provide a cursory mention of trust by incorporating a single quote to elicit contribution. In addition, few extrapolated on the passages provided or sought to fully contextualize trust within the body of the text.

In contrast, nearly every article filed as providing substantive content for a trustrelated concept thoroughly discussed at least one of the concepts provided. Many of these articles provided short passages or a series of quotations from focus groups and interviews to substantiate these themes, but many of these articles identified an unannounced primary theme around which the rest of the article focused. A list of articles providing substantive and cursory discussion on trust and trust-related concepts can be found in Appendix F. In addition, further breakdown of the types of trust-related concepts discussed by article can be found in Appendix G.

Review of these data revealed that every article discussed at least one trust-related concept in a cursory fashion, with communication being discussed in almost every article. Of the 51 articles, $84 \%(n=43)$ of the reviewed articles discussed communication, of which $45 \%(n=19)$ discussed communication in a substantive manner. The second most 
frequently referenced concept was cultural competence $(n=21)$, followed by comfort $(n$ $=19)$ and understanding $(n=19)$.

\section{Discussion}

\section{Summary of Evidence}

\section{Cochlear Implants}

In total, 1,925 citations were identified using the electronic database CINAHL and performing reference list searches in related articles. Of the 106 articles deemed eligible for review, 93 were assessed for their relevance to trust and trust-related concepts in the nursing and allied health literature from the perspectives of Deaf people, but only 51 articles met the eligibility criteria after a full-text review was completed. Of the 42 articles deemed ineligible after review, several were removed for non-cochlear implant related foci, but the disproportionate number that were removed for their focus on cochlear implants warrants deeper consideration.

One possible reason for the elevated number of citations identified as addressing cochlear implants might be because CINAHL retrieves citations from allied health programs which frequently interact with people using cochlear implants. Examples include speech pathologists, audiologist, and some medical professionals. Another reason might be because only one exclusion rationale was recorded for removing each citation. As such, some citations may have had multiple exclusion rationale, but the single reviewer may have shown an unconscious bias to file citations meeting multiple exclusion criteria under 'cochlear implant' rather than one of the other exclusion categories. However, these potential justifications do not account for the volume of citations pertaining to cochlear implants when compared to the number of citations 
addressing Deafness from a cultural perspective. This contrast suggests that even among the nursing and allied health sciences greater research effort and emphasis is placed on pathologizing deafness than seeking to understand individuals who are Deaf.

\section{Trust}

Despite being a prominent area of research in health literature (Ozawa \& Sripad, 2013), trust in healthcare settings, institutions, or personnel is rarely mentioned or discussed in relation to people who are culturally Deaf. Of the 1,925 citations identified, 11 were retrieved using the search term trust, and almost half of these $(n=5)$ were inappropriately identified. In addition, after expanding the search for articles discussing trust from a Deaf perspective within the nursing and allied health literature, only an additional 18 were identified (including articles with either substantive or cursory mentions). In comparison, using the same search terms, more than 12 different trustrelated concepts were identified and discussed. This evidence suggests that among the healthcare literature focused on deafness and Deaf people, the concept of trust lacks conceptual clarity.

\section{Patient-Provider Relationships}

Though not recorded in the DCF several additional trends were noted in the articles retrieved for full-text review. First, very few of the articles directly investigated the patient-provider relationship. Instead, most articles addressed the general experiences of Deaf patients in healthcare settings and mention PCPs in passing. In addition, the majority of healthcare interactions described, and healthcare settings discussed, involved mental health services. Though psychiatric treatment is similar in many ways to primary practice, it is curious that so few articles were found to focus on primary care in a more 
traditional sense. Future investigations should include data on the setting of the patientprovider relationship in case the perspective of trust is influenced by the setting.

\section{Limitations}

This scoping review has several limitations. First, only one investigator charted the data. Therefore, no additional researchers contributed to a 'quality check' of the results. In addition, only one rationale was selected for excluding each citation. As such, additional conclusions might be made regarding the literature with more data. Similarly, only one database was used to compile the citations. Although this scoping review sought to summarize the literature surrounding trust within patient-provider relationships from the perspective of Deaf people within the nursing and allied health fields, findings regarding the conceptualization of trust and trustworthiness in the patient-provider relationships cannot be generalized to all databases due to the narrowed parameters of the CINAHL database.

\section{Conclusions}

This scoping review provides an overview of the literature on trust within patientprovider relationships from the perspective of Deaf people in the nursing and allied health arenas. From the 1,925 citations identified, only 11 discussed patient-provider trust from the perspective of Deaf people, in a substantive manner. In comparison, 43 articles discussed communication. As such, little consensus appears to exist regarding patientprovider trust in this population, supporting the need for further exploration on the topic of trust through from the perspectives of culturally Deaf people. 
CHAPTER III

\section{METHODOLOGICAL CHALLENGES AND STRATEGIES \\ FOR CROSS-CULTURAL RESEARCH IN ASL}

\section{Introduction}

Centuries of scholarly publications about Deaf people, their educational needs, and research methodologies exist, with the longest running journal dedicating more than 170 years to the state of the science (American Annals of the Deaf, 2020). Among these publications, many early and mid-twentieth century works misrepresent sign language and its users by inappropriately 'pathologizing' deafness and supporting a culture that boasted the superiority of individuals who hear over those who do not (Bauman, 2004; Myers \& Fernandes, 2010). However, the influences of the French philosopher Jacques Derrida (Bauman, 2004) and others (Myers \& Fernandes, 2010) transitioned the Deaf narrative from one of hearing superiority to one of cross-cultural exploration. From here, publications emphasizing ethical considerations in cross-cultural research with members of Deaf communities gained traction. Researchers like Pollard (1992), Harris et al. (2009), and Wolsey et al. (2017) all emphasize the need for community member integration in research projects and the investigation of culturally valued research results. However, few provide insight into the challenges experienced by researchers conducting studies with people who are Deaf, even when cross-cultural guidelines are followed. Therefore, the purpose of this paper is to describe unexpected events that occurred, and strategies used to address these events while, conducting a qualitative study with 
members of Deaf communities. This paper seeks to highlight the importance of following cross-cultural best practices for their practicality and provide evidence for how even the 'best laid schemes, [still go awry]' (Burns, 1785, as cited in Poetry Foundation, 2020).

The study on which this manuscript is based investigated the conceptualization of trust and trustworthiness towards primary care providers, from a Deaf perspective using a qualitative descriptive design. Data were obtained from a focus group of nine Deaf participants conducted in American Sign Language (ASL) and one phone interview conducted in spoken English. Prior to conducting the study, I increased my involvement with the local Deaf community, took classes to improve my ability to communicate in ASL, and completed an unrelated qualitative study (in English) as a primary investigator. The results of an initial literature review referenced the importance of addressing ethical guidelines when conducting research with members of Deaf communities (Harris et al., 2009; Pollard et al., 1992; Wolsey et al., 2017), but articles discussing methodological considerations were not identified. Some articles provided valuable (though often incomplete) insight in their method sections. For example, focus group size and facility accommodation guidelines were obtained from Balch and Mertens (1999) and information about informed consent construction and delivery were obtained from McKee et al. (2013). However, these articles do not provide examples of the challenges encountered while conducting cross-cultural research in ASL. Therefore, this paper presents key challenges concerning the consultation of, and engagement with, members of the Deaf community during cross-cultural research. Moreover, this paper specifically focuses on the importance of incorporating Deaf individuals in cross-cultural research and unforeseen challenges that arise when working with interpreters. 


\section{Findings}

\section{Deaf People}

It might go without saying that engaging members of your target population prior to implementation is beneficial, but their influence cannot be overstated. During the development, implementation, and analysis of this study, the lack of sustained involvement by Deaf people resulted in stagnation, confusion, and redundancy. It was only through the engagement of Deaf people that the project progressed.

Initially, Deaf individuals were tangentially consulted regarding the general purpose and desired outcome of this study. The overwhelming majority encouraged my interest, 'the healthcare experiences of Deaf people', but erroneously assumed my goal was to teach cultural competence to providers. Later, Deaf and interpreter faculty members inside the university, and outside the university, were consulted to contribute with little success. Eventually, a university employee within the ASL department (code name: Louis) became the project's unofficial 'cultural broker' due to his continued interest, availability, and desire to contribute.

A cultural broker is a person who acts as a mediator between members of the research team and the cultural group being investigated (Jezewski, 1993). This person is typically a member of the cultural group, instilling trust within the community, but when this person is also a member of the research team their role can be expanded to include more efficient content creation, recruitment, data collection, and data analysis. Several researchers use official cultural brokers when conducting research with members of Deaf communities (Cabral et al., 2013); however, my study lacked an official cultural broker. As such, the lack of cultural broker presented challenges in creating and distributing 
recruitment material, as well as in data analysis. Though Louis was not legally permitted to assist in several of these facets, he was able to provide valuable insight into Deaf culture which aided in the project's success.

\section{Deaf-friendly Recruitment Material Creation}

Apart from technical challenges inherent in filming and editing digital recruitment material in a 'Deaf-friendly' manner, writing and performing a script was fraught with complications. First, all English material needed to be translated into ASL (see Legal Interpreting below). Then, the translation needed to be performed and recorded. Given that I was conversational in ASL, I attempted the performance. However, review of the material by Louis revealed several errors in my performance. To address this problem, Louis acted as my speech coach, script supervisor, and director by correcting my grammar, performing my lines behind camera like a teleprompter, and approving each take. A better approach would have been to involve Louis in the creation of all recruitment material. His contribution would have been invaluable when creating a culturally desirable script, and if willing, he could have performed the script himself after undergoing the necessary training and IRB approvals to become a member of the research team.

\section{Recruitment}

I initially distributed flyers per the recruitment strategies approved by the IRB. These flyers, written in English, were distributed to members of the Deaf community at community events. During these events, I also communicated directly with members of the community, explaining the study and providing them with the opportunity to indicate their interest in participating by completing an electronic screening questionnaire at a 
later date. After several attempts spanning approximately two months, only four individuals completed the screening questionnaire, and none returned correspondence to schedule an interview or focus group. Later, I attempted to advertise electronically through Facebook. Electronic advertising permitted a direct link to the screening questionnaire, thus avoiding the need to type the provided URL into a web browser. This alteration yielded minimal additional traffic to the screening questionnaire and no additional screening questionnaire completions. After Louis viewed the Facebook post, he shared it with his friends and family and a surge of potential participants accessed the site. Initially, this increase in traffic yielded only a few additional contacts, all of whom lived out-of-state individuals and unable to attend an in-person focus group or interview. However, over the course of two months Louis' dissemination of recruitment material, yielded additional traffic which ultimately led to the identification of an adequate number of participants.

\section{Data Analysis}

Data for this study were analyzed in English. Audio recordings from the focus group and interview were transcribed and video recordings from the focus group were used to verify the relative accuracy of translations and attribute specific comments to their respective participant. This analysis of transcribed data did not pose any additional problems beyond those of traditional qualitative analysis. However, conducting an analysis in English required additional steps, including English transcript verification and continued checks for coding authenticity. Had a Deaf person been a member of the research team, they could have coded the material in its original form faster than the content was transcribed and verified by the non-Deaf researcher. In addition, working in 
the participant's native language would have provided more data to analyze as nuances contained in facial expressions and gestures may not have made the translation.

\section{Interpreters}

Three interpreters were used during the study. Peter translated the screening question material, including the preamble, while Laverne and Shirley co-interpreted during the focus group (all pseudonyms). No interpreter was employed during the phone interview conducted in English. All three interpreters were provided with reference material before their involvement: Peter received the Screening Questionnaire and preamble; Laverne and Shirley received the Informed Consent Form and interview script. Peter used a webcam to independently film and share his preamble translation in ASL. In contrast, Laverne and Shirley were not directly recorded during the in-person focus group. Instead, cameras periodically recorded Laverne and Shirley in the periphery while capturing participants arranged in an open trapezoid formation (see Appendix H). The use of interpreters provided two broad categories of challenges: Establishing Linguistic Equivalence and Using Interpreters.

\section{Establishing Linguistic Equivalence}

Unless an interpreter is familiar with the focus of the study and its methodology, the message may be inaccurately converted if the interpreter inappropriately emphasizes the wrong style of equivalence. For example, semantic equivalence is the idea that two words from different languages (e.g., English and French) contain the same meaning (Ervas, 2014): dog (English) vs. chien (French). The use of semantic equivalence is often contrasted with pragmatic (Ervas, 2014), dynamic (Nida, 1964), or functional equivalence (Jin \& Nida, 2006) in which the focus of the translation is placed on the 
effect the message has on the receiver (Brannen, 1993). Functional equivalence is most evident when translating idioms like the English expression 'to butter someone up' into the French form 'passer de la pomade á quelqu'un'. In both languages the underlying message is to make the other party feel good in hopes of receiving a favor, but depending on your preferred back-translation, the literal form 'pass of the ointment to someone' doesn't have the same meaning in English. However, elements of functional equivalence can also be seen in non-figurative translations when words hold different functions for grammatical reasons (Ervas, 2014). Likewise, cultural equivalence, an equivalence style akin to functional equivalence (Higashino, 2001), emphasizes the use of culturally congruent vocabulary - including a change in form when original meanings would be lost or misconstrued (de Waard \& Nida, 1998): subsidized housing (English) vs. logement social (French). During this study, examples of ineffective English to ASL conversions included the terms 'screening questions', 'integrity', 'benevolence', and Dr. Gregory 'House' (of television fame). In each of these cases, interpreters chose ASL words or phrases corresponding with the original text, but their adherence to an ineffective equivalence style created challenges during data collection.

Screening Questions. Screening questions are those provided to potential participants to determine their eligibility before offering study inclusion. This term appeared in the introductory videos to the study and was translated directly from the IRBapproved recruitment material. The interpreter translated this term literally by first signing a variant of the sign 'SCREEN' (a semipermeable, upright partition) immediately followed by repeating the sign 'QUESTION' (directed at the audience). I was informed by Louis that, in ASL, this sentence illogically translates to 'a screen that asks the 
audience questions.' Louis emphasized that the translation can be deciphered in context, but the message is not clear due to grammatical differences between verbal and signed languages.

The English word 'screen' is a homonym, while the relevant form (i.e., 'screening') is either an adjective (e.g., 'screening' questions) or a gerund (e.g., questions used for 'screening'). In both cases, the participle form of screen (i.e., screening) no longer functions as a verb. S-C-R-E-E-N (fingerspelled in ASL) is also a homonym, used for both door screens and computer monitors. However, the signed versions of both are distinctly different. In addition, neither version of SCREEN (signed in ASL) is an adjective or a gerund because ASL does not permit this part of speech (though some participles do exist). Therefore, it is best to convey the concept of screening questions by describing what will occur without using the term. An alternative is to initially fingerspell the word, then describe the concept, and then finally provide a culturally congruent manual sign that accurately conveys the concept in a sentence. Regardless, using a semantically equivalent approach to convert research jargon may be ill advised.

Integrity and Benevolence. As previously noted, this study was guided by the Proposed Model of Trust (Mayer et al., 1995) to conceptualize the perceptions of trustworthiness as experienced by people who are culturally Deaf. As such, the 'factors of perceived trustworthiness' provided in the model were key concepts in need of exploration: ability, integrity, and benevolence. A lack of semantic equivalence between the English words integrity and benevolence and their ASL counterparts was problematic.

First, ASL is not a non-spoken variant of American English, despite using several English words to convey linguistically challenging or foreign concepts. Like German 
words in English (e.g., zigzag), words that are fingerspelled in ASL are almost exclusively loanwords, but unlike most English loanwords, most ASL loanwords require the user to have access to an unnecessarily elevated lexicon. For example, some English to ASL loanwords appear to have full ASL integration (e.g., W-H-E-N), while other English words are fingerspelled to add clarity or specificity (e.g., fingerspelling V-A-N instead of signing CAR). Some English words have been lexicalized to create new words in the target language (e.g., R-E-F - delivered with unique hand and mouth gestures - for refrigerator). Other English words are spelled to add emphasis or personal flare in conversation. An English equivalent might be over-pronouncing the word 'résumé' in one's best French accent. However, loanwords conveying descriptions are infrequently used in ASL. English words like contiguous, callipygous, and cacophony describe very distinct and complex concepts; however, their meanings are conveyed clearly, and often in more entertaining ways, without a formal ASL sign or use of a loanword. Consistent with this trend, the English words 'integrity' and 'benevolence' do not exist in ASL.

A counter example would be the word 'ability'. Abilities are often evident and easily portrayed through actions. As such, ASL is equipped with two semantically equivalent signs for the English word 'ability', depending on the preferred meaning. However, 'integrity' and 'benevolence' are both used to describe intrinsic qualities. Though a person's actions are inextricably linked to these attributes, actions themselves are not examples of the attributes. Actions are indirect measures of these intrinsic attributes, and ASL appears to be less conducive to expressing intrinsic attributes. Moreover, the difficulty participants had identifying and relating to the terms support the 
Sapir-Whorf hypothesis of linguistic relativity: A person's language influences their perceptions (Lucy, 2015).

During the focus group, the interpreters used the sign 'HONESTY' as a proxy for 'integrity'. Though honesty is a part of integrity, the sign, which shares semantic equivalence with its English counterpart, fails to capture the moral and principle aspects of integrity. When asked to distinguish the two terms the entire group was not able to do so, and further description of the term did not improve clarity. A variant combining 'HONESTY' and 'INSIDE' exists (Lapiak, J., 2020b) but did not provide clarity. This prompted Laverne to tell a story about finding $\$ 20$. She emphasized the actions taken by the participants would indicate the participants' integrity: "DO WHAT? PUT-INPOCKET? LEAVE-IT? WHICH? NOT YOUR MONEY ... THAT ‘I-N-T-E-G-R-I-TY'”. Storytelling like this was effective in opening dialogue about the topic at hand but use of examples was not without complications.

Dr. Gregory House. The word 'benevolence’ also lacks a semantically equivalent ASL sign, though functionally equivalent versions can be found (Lapiak, 2020a). During the focus group, the interpreters fingerspelled benevolent, but more frequently signed 'NICE'. Lack of equivalence aside, many members of the focus group failed to grasp the relevance of the question. To help illustrate the absence of benevolence in healthcare, while retaining one's ability and integrity, the TV character Dr. Gregory House, was referenced. The interpreter signed, "YOU KNOW H-O-U-S-E? HOUSE." Most of the older focus group participants were acquainted with the television show and required no additional clarification. However, other focus group participants failed to identify that 'HOUSE' was a name, not a physical building. Several possible 
reasons exist for this confusion, but this example highlights an unexpected challenge when relying on poorly established loanwords in ASL. A better alternative might be to work with the interpreter before commencing interviews to identify and prepare the most effective translations and examples.

\section{Using Interpreters}

Following the recommendations of Sheppard (2011a), an attempt was made to locate interpreters with a minimum of five years of experience interpreting in related content areas. The interpreters identified each had more than eight years of experience with general interpreting, and one interpreter had research interpreting experience that did not include focus groups. Though this study did not focus on healthcare procedures or healthcare terminology, both Laverne and Shirley predominantly interpret in healthcare settings with medical interpreting certifications, and both Peter and Laverne worked with me in the past. With this in mind, several unexpected events occurred while obtaining interpreters, analyzing data from the interpretations, and establishing the interpreters' role in the study.

Legal Interpreting. No legal interpretation was performed during this study; however, the preamble and Informed Consent Form contained language reminiscent of legal documents. During the creation of the Screening Questionnaire, I struggled to find an interpreter willing to translate the preamble for fear their commissioned service might violate their license as a general interpreter (legal interpreting requires additional training and certification). I was able to convince Peter, an interpreter with whom I've maintained a semi-professional relationship with for several years, to provide a translation with the agreement that I 1) not reveal their identity, 2) use the video-recorded translation as a 
template to build my own translation, and 3) pay a discounted rate for their service. In contrast, legal interpretation did not appear to extend to the interpreters during the focus group in that no discussion occurred regarding their willingness (or lack thereof) to interpret my words as I read from the Informed Consent Form. The difference between the reaction of interpreters to the preamble and the informed consent may be related to differences in scope of work. When interpreting my words, Laverne and Shirley were operating under their license. When asked to translate a written document, Peter was not.

Choice of Linguistic 'Register'. Register is one of several linguistic devices used to typify language users. This combination of vocabulary, punctuation, and grammar signal formality with five, generally accepted, distinct categories: high formal, formal, neutral, informal, and vulgar (Haines, 2020). The choice in register can signal many things including a person's occupation (Trudgill, 1983) and status within a group (Agha, 2007). It is important for ASL interpreters to understand and appropriately use register while converting between languages to ensure the individuals accurately represented in the target language (Shaw, 1987).

During this study, Laverne and Shirley used different registers. Laverne used an informal register, supported by a truncated vocabulary, while Shirley used a formal register, supported by more eloquent speech patterns. As an example, Laverne converted the sign 'THAT++' (i.e., 'THAT' repeated for emphasis of agreement) into the English expressions "That!" or "Exactly!" while Shirley converted the same sign into the English expression "I would agree". Different use of registers might be warranted if different participants used different registers or if any participant switched between registers. However, change in register was particularly problematic when both interpreters took 
turns interpreting for the same person. In these cases, the switch between linguistic registers made thematic and analytic coding challenging. In addition, an unwarranted change in register might make other forms of (unused) coding challenging, if not entirely impossible (e.g., semantic analysis).

Choice in Role. The Code of Professional Conduct provided by the Registry of Interpreters for the Deaf (2005) explicitly states that interpreters are to "[r]efrain from providing counsel, advice, or personal opinions" while interpreting (p. 3). Interpreter training programs and agencies generally interpret the Code of Professional Conduct conservatively, meaning interpreters should be a 'machine': only able to convert languages and unable to assist in other ways (Baker-Shenk, 1992). However, some interpreters do not subscribe to the machine model of interpreting (Baker-Shenk, 1992). These interpreters acknowledge the power dynamics in each situation and react to these power dynamics as 'allies', empowering the Deaf person by correcting the imbalance (Baker-Shenk, 1992). During the focus group, Laverne and Shirley appeared to ascribe to different interpreting models.

Laverne was quick to interject and assist me (the focus group facilitator) by offering helpful alternatives to my questions or statements that lacked ASL equivalence. Shirley appeared less willing to assume this role, interpreting to the best of her ability without comment. If I said something illogical, both would interpret my words, but Shirley would not ask for clarity unless a participant explicitly asked. In this way, Laverne acted as an ally, wanting to ensure effective communication was established, while Shirley acted as a machine, wanting to ensure the truest version of each message was delivered. Ironically, the interpreter role assumed by both interpreters seemed at odds 
with their choice in registers: As an 'ally', Laverne used a register more congruent with participant comments; as a 'machine', Shirley, used an elevated register by converting single gestures into elegant commentary.

Interpretation Preferences. Advantages and disadvantages exist for both the 'machine' and the 'ally' role of interpreting. For example, one Deaf participant later informed me that they preferred Laverne's interpreting style over Shirley's because Laverne's style felt more authentic. For example, during the focus group, Laverne literally mimicked a participant by shrugging and saying "Ugh..." rather than attempt an English translation. In contrast, Shirley's interpreting style was more verbose, and her loquacious interpretations provided more data and greater nuance for analysis.

\section{Recommendations}

\section{Team-Up with Deaf People}

Ethical issues aside, conducting research in ASL and among members of the Deaf community presents many challenges. Taking a community engagement approach, including these individuals into the research decision making process can improve the oft-strained relationship between researchers and people who identify as culturally Deaf (Meador \& Zazove, 2005). In addition, community engagement approaches build needed partnerships between underserved communities and funding agencies (Agency for Healthcare Research and Quality, 2009). By engaging members of the Deaf community, these partnerships can grow, alleviating many of the challenges described above.

\section{Establish Yourself in the Community}

Sheppard (2011a) expressed the importance of establishing yourself among ASL interpreters who might act as gatekeepers, facilitating or hindering your research efforts. 
In my case, I felt well established in the local Deaf community and among local interpreters. As such, I did not notice any effort to hinder my study by either group, though several interpreters felt it would be inappropriate for them to use their position to disseminate information about the study on my behalf. In contrast, some individuals, many who only knew me through other members of the Deaf community, expressed interest in my study and a willingness to share it with others. Unfortunately, this did not result in enhanced recruitment due to the complexity of the implemented recruitment strategy. In particular, the implemented recruitment strategy required potential participants to read an English flier and manually transfer a multicharacter URL into a web browser, something that was later overcome by gaining IRB approval to use electronic forms of recruitment with embedded hyperlinks.

\section{If Possible, Forgo Interpreters}

Sheppard (2011a) recommends negotiating with interpreters, quoting a range per hour with variants in travel ('portal') fees, billing increments, and minimal duration of services. My experience with interpreters demonstrated a flat rate of $\$ 55$ per hour with a one-hour portal fee, and quarter-hour billing after the first hour. This price was quoted to me by both individuals and agencies, with Peter willing to accept a lower wage in exchange for anonymity. As such, the use of a focus group for data collection assisted in minimizing costs, but having an interpreter as a trained, fulltime research partner would have been financially unfeasible. One possible solution is to find interpreters who also hold faculty positions in search of scholarship opportunities. These individuals might be willing to engage in research projects for non-monetary remunerations such as authorship. Moreover, though the value of interpreters cannot be understated, the allotted 
money may be more equitably distributed by hiring and training someone who is Deaf. In this way, the individual would have the opportunity to gain research experience and profit from their abilities rather than subverting members of the Deaf community.

This is not to say interpreters should never be used. Depending on the researcher's ability to communicate in ASL, and more importantly, the willingness of people to work through any communication barriers, an interpreter might be needed while preparing an individual who is Deaf for their role as a research team member. Likewise, ASL interpreters should be used if hearing researchers want real-time feedback while observing facilitators and participants who use ASL (Singleton et al., 2014). In addition, an ASL interpreter should be used if a signing facilitator is not used and the facilitator lacks the ability to fully communicate in ASL (Singleton et al., 2014). Finally, if data will be analyzed in English, and the researcher lacks an alternative means of converting the transcript from ASL to English, interpreters should be hired to perform the task.

\section{Work in ASL}

Pollard (2002) stresses the importance of conducting research in the participant's 'natural' language. However, it is also important that the facilitator be able to communicate directly with participants (Singleton et al., 2014). For some culturally Deaf adults, written or spoken English may be acceptable options, but the language choice and ability of a facilitator to communicate through the chosen modality should be determined by the participant prior to attending an interview or focus group. If the facilitator is not the primary researcher, the facilitator should be trained to conduct the research independently, only reporting back to the primary researcher when necessary (Plumridge et al., 2012). Likewise, some scholars indicated ASL should be the 'lingua franca' (i.e., 
language of choice) for any research team with contributors who sign (Wolsey et al., 2017), though the extent of this recommendation may be situational depending on levels of involvement and communication preferences. Regardless, data should be coded in ASL, leveraging technology to visually identify themes and preserve as many nuances in the data as possible (Anderson et al., 2018). Only after identifying themes should data be translated to English for other team members to appraise and future researchers to understand.

\section{Conclusion}

In this manuscript I provided examples of specific challenges experienced while conducting research in ASL, with members of the Deaf population. Solutions to these challenges and recommendations for researchers planning to conduct cross-cultural research are also provided. Challenges faced range from research design to execution and focused on pragmatic issues in language conversion and interpreter use. Finally, I provided recommendations to help others avoid similar mistakes. These recommendations included increasing linguistic diversity with the research team, prolonged engagement with Deaf communities, less reliance on interpreters for essential research activities, and conducting studies solely in ASL (including data analysis). 


\section{CHAPTER IV}

TRUST IN PRIMARY CARE PROVIDERS:PERSPECTIVES FROM DEAF PERSONS

\section{Introduction}

\section{Background and Significance}

Trust is an important measure in patient-provider relationships. High levels of trust support better patient outcomes by increasing a patient's willingness to participate in preventative services (O'Malley et al., 2002; Pellowski et al., 2017), adhere to treatment regimens (Blackstock et al., 2012; Schoenthaler et al., 2014; Tran et al., 2017), not seek alternative providers (Hall et al., 2002), and be health literate (White et al., 2013). However, several cultural groups are known for having low levels of patient-provider trust, or high levels of mistrust (Murray \& McCrone, 2015). The preponderance of literature on the topic target Black/African American and Hispanic communities, with members of other cultural groups (e.g., Asians and Native Americans) loosely referenced in demographic tables (Jacobs et al., 2011). These studies rarely provided additional demographic information on the hearing status of participants, and due to common recruitment practices at the time, were unlikely to include deafened individuals in their sample (Barnett \& Franks, 1999).

Deaf people exist as a unique subset of American culture (Reagan, 1995). Deaf people, those who identify as 'Deaf' with a capital 'D', share similar life experiences, hold similar beliefs, speak the same language, and take pride in their heritage (Kaplan, 1996). Though Deaf culture is not monolithic, the historical oppression experienced by 
members of the Deaf community (Lane, 1999), may influence members of the Deaf community to have difficulty developing trust in healthcare providers. In addition, the continued lack of cultural competence within healthcare and healthcare-related fields (Anderson et al., 2017b; Ferguson-Coleman et al., 2014; Kaplan, 1996; Mprah, 2013) may further exacerbate the strain between these two cultures. Yet, a recent search of the healthcare and allied health fields literature revealed a lack of publications dedicated to understanding patient-provider trust from the perspective of people who identify as culturally Deaf.

The predominant theme within the Deaf-focused, healthcare literature surrounds barriers to care (Anderson et al., 2017a; Bat-Chava et al., 2005; Mprah, 2013; Reader et al., 2017; Thu \& Huang, 2014), general experiences in healthcare settings (Anderson et al., 2017b; Frank, 2017; Steinberg et al., 2002), and advice for healthcare providers caring for patients who identify as Deaf (Meador \& Zazove, 2005; Middleton, Turner, et al., 2010; Nonaka, 2016; Orrie \& Motsohi, 2018; Stebnicki \& Coeling, 1999). Though these publications are important to building a robust knowledge base detailing the intersection of healthcare and Deaf culture, few publications discuss the cornerstone of this intersection: trust. Instead, many references the concept of trust parenthetically (Maddalena et al., 2012), or assert a lack of trust without citation (Ferguson-Coleman et al., 2014; Jampel, 2010; Meador \& Zazove, 2005). Even in qualitative studies where healthcare experiences are discussed, trust is not commonly referenced by participants or discussed by researchers (Maddalena et al., 2012; Steinberg et al., 1998). As such, the purpose of this study was to explore the concepts of trust and trustworthiness as they relate to Deaf peoples' experiences with Primary Care Providers (PCPs). 


\section{Theoretical Framework}

This study was guided by the Proposed Model of Trust (PMT) as developed by Mayer et al., (1995). This model seeks to explain the feedback loop associated with an individual's level of trust (i.e., perception of trustworthiness) towards, and subsequent behavior with, an organization (see Appendix A). The PMT is applicable to patientprovider trust through the looped, linear relationship of the four core components: Perceived trustworthiness, trust, risk, and outcomes. This study focuses on the factors of perceived trustworthiness, trust and their moderating variable (i.e., trust propensity) as they are experienced by people who identify as culturally Deaf in patient-provider relationships (see Appendix B).

\section{Trust vs. Trustworthiness}

Though trust and trustworthiness may appear to be used interchangeably they are two different concepts. Trust, as it is defined for the purpose of this paper, is the willingness to be vulnerable to another person (Mayer et al., 1995). This willingness does not imply an individual will accept and actively take part in the inherent risk of trusting others, merely that an individual is willing to consider a specific course of action. In contrast, trustworthiness is a composite of attributes that, when combined, informs an individual whether someone else can be trusted (Mayer et al., 1995). In this way, trustworthiness is a measure of someone's ability to be trusted, as perceived by the individual doing the trusting (the 'trustor'). Trustworthiness is a personal characteristic given to someone by another, while trust is a personal decision an individual makes about themselves. 


\section{Research Design and Methods}

This study used a descriptive, qualitative ethnographic approach to investigate the experiences of individuals who are culturally Deaf. Using a predetermined set of questions, data were combined from a face-to-face focus group conducted in American Sign Language (ASL) and a one-on-one phone interview conducted in English to reveal unifying cultural experiences and perceptions about healthcare culture.

\section{Setting}

Focus group data were collected in Louisville, Kentucky at the University of Louisville's School of Nursing in a private classroom. The focus group was held in one of the building's lower classrooms, on a Friday night, after traditional course hours. In accordance with recommendations from Balch and Mertens (1999), the room had minimal visual distractions such as mirrors and had no windows to ensure privacy. Participants were originally arranged in a circular orientation without tables, but with the introduction of snacks and refreshments, the room was rearranged in a trapezoid formation, with no more than three participants on any of the sides, to incorporate tables (see Appendix H). The recording equipment, facilitators, and interpreters were arranged in a palindrome at the base of the trapezoid. One audio recorder was positioned on a stool in front of each interpreter and both video recorders were oriented to maximize data capture from participants, with interpreters occasionally observable in the periphery.

\section{Sample}

A relatively diverse set of participants from the local Deaf community were invited to participate. All participants $(\mathrm{N}=10)$ were pre-screened for eligibility using the following self-reported criteria: Age of majority, cultural Deafness, use of ASL as 
primary (or preferred) means of communication, previous experience with a PCP, and the ability to participate in the interview for the required amount of time (i.e., two hours for focus groups and one hour for interviews). Participants were not excluded for use of English or assistive devices including cochlear implants. Participants were purposefully selected in an effort to maximize diversity, although greater uniformity existed in their onset of deafness, lack of cochlear implant use, gender, and location of residence than idealized. The sample included a wide range of races/ethnicities, ages, education, and style of primary and secondary schooling attendance (see Appendix I).

\section{Measures}

\section{Demographic Questionnaire}

An 11-item demographic questionnaire was administered to all participants. The questionnaire covered general questions about age, race, and gender, but also included frequently reported demographic information pertaining to Deaf people including age of onset for deafness, cultural identity, and the types of schools attended for primary and secondary education.

\section{Interview Guide}

One interview guide was created for both focus group and one-on-one interviews. Originally, nine open-ended questions were derived from the theoretical framework (see Appendix A) with a specific focus on the components most relevant to uncovering cultural perceptions of trustworthiness with respect to healthcare providers (see Appendix B). However, keeping with the iterative nature of qualitative studies, participant responses to interview questions generated additional questions and topics of conversation mid-interview. For example, during the focus group, two of the questions 
were converted to Likert-style questions, prompted by participant request, due to the time constraints and the difficulty with which participants experienced previous open-ended questions.

The first three questions focused on general conceptualizations of trust including trust and related words. The next two questions focused on trust as an internal construct. For example, does trust change when discussing different people and how the knowledge of its experience is understood. The four remaining questions and their follow-up questions focused on trustworthiness as it pertained to PCPs. Using the PMT as a framework (Mayer et al., 1995), participants were asked about the relative importance of ability, integrity, and benevolence in the patient-provider relationship. The questions 'How trustworthy are PCPs?' and 'How does the trustworthiness of a PCP change if they are hearing or Deaf?' were converted to Likert-style questions.

Throughout the focus group, participants were encouraged to consider focusing their discussion on all types of PCPs, but due to cultural norms and linguistic vernacular most participants specifically discussed their experiences with physicians. As such, prepared prompts incorporated this language in an effort to obtain richer data.

\section{Procedure}

\section{Recruitment}

Participants were recruited using a combination of convenience and snowball sampling methods (Patton, 1990), with purposeful sampling used to finalize participant selection. First, recruitment material (i.e., study introduction, signed preamble, and screening questionnaire) was translated into ASL and uploaded to Google Forms. Then, fliers encouraging potential participants to access the online material were distributed at 
local Deaf events. Later, an electronic announcement directing potential participants to the same Google Form were created and distributed on Facebook. Successful completion of the Google Form provided self-reported potential participant contact information to the researcher for scheduling participation.

\section{Data Collection}

A private focus group discussion was chosen as the preferred means of data collection to minimize cost by hiring one interpreter for up to five participants and to allow participants the opportunity to extrapolate on the ideas of others, rather than be self-reliant when reflecting on abstract concepts. A singular one-on-one phone interview was also completed with one participant who was unable to attend the focus group. The focus group was conducted in an unoccupied classroom at the University of Louisville's School of Nursing. The phone interview was conducted over the university's secure wireless network in a vacant, private office, using the phone application 'TapACall Pro' (Epic Enterprises LLC, 2019).

Prior to offering study inclusion, all participants were screened and provided informed consent forms. Before providing written consent, licensed ASL interpreters translated for the researcher as the researcher read the informed consent form to the potential participants. For the phone interview, no translation was required as the participant's ability to speak and hear English was sufficient to provide consent without an interpreter. In addition, due to the physical distance between the participant and researcher, written consent was provided via an electronic signature, prior to enrollment.

Two weeks before the focus group, two licensed ASL interpreters, each with more than eight years of general interpreting experience and certifications in medical 
interpreting were scheduled through an agency. Only one interpreter had experience interpreting for research study participants. Both were provided with study material in advance, including a copy of the informed consent form, demographic questionnaire, and study questions. Contrary to the procedures of Sheppard (2011b), participants and interpreters were given general information about, but not the identity of, the other participants and interpreters. This change in protocol was chosen to avoid favoritism during the focus group and was deemed acceptable because no protected health information was discussed.

After providing written consent, participants were asked to complete and return the demographic questionnaire before the electronic equipment started recording. During this time, participants were able to ask the interpreters and the facilitators for assistance. After all demographic questionnaires were collected, the video and audio recorders were turned on, and the facilitator started the discussion using the interview guide.

\section{Data Management}

All physical data including informed consent forms and demographic questionnaires were transferred from the focus group site to a locked cabinet, behind a secure locked door, using a lockbox. Data from demographic questionnaires were entered into an electronic spreadsheet and transferred with the digital video and audio recordings to a password-protected server using a password-protected internet connection. After verifying the electronic transfers, the original digital data were deleted. The audio recordings from both focus group and the individual interview were transcribed by the researcher; the video recordings were used later to verify the accuracy of the transcripts. 
Any additional content, such as field notes, were electronically created and transferred to the password-protected server.

\section{Data Analysis}

Analysis of data was an iterative process. Descriptive statistics were calculated using mental math and transcripts were manually coded using Word (Microsoft, 2019). Following the recommendations of Polit and Beck (2012), qualitative analysis of the transcripts initially focused on thematic analysis, identifying three primary categories in which data seemed to reoccur. These categories were then compared and contrasted with the theoretical framework to provide context and identify concepts in need of further investigation. Additional rounds of analytic coding were used to further solidify categories into themes, collapsing several categories into multiple themes and identifying overarching themes.

\section{Rigor}

The trustworthiness of data was addressed by using member checks (Lincoln \& Guba, 1985). During the focus group, participants were asked to confirm the facilitator's understanding. Additional member checks were employed during data analysis by contacting participants to ensure the opinions of these participants were accurately represented in the findings.

The authenticity of data was established by developing and maintaining a trusting relationship with members of the local Deaf community and several of the participants for more than five years prior to starting the study (Manning, 1997). Authenticity was also established by maintaining a congenial and collaborative environment during the focus group. 
The quality of data was upheld by providing clear and defined inclusion criteria and reviewing both protocol and data with mentors from their respective areas (Krueger \& Casey, 2009). Quality was also upheld through the use of a theoretical framework and the future dissemination of findings among members of the Deaf community.

\section{Results}

\section{Demographic Characteristics}

A total of ten individuals participated in this study. The majority of participants self-identified as male, White, culturally Deaf, and deaf at birth. At least one participant self-identified as being a member of each age category and nearly half of the participants had obtained a post-secondary degree.

Half of the participants, including members from each age group, were exclusively educated in Deaf schools. Three were exclusively educated in hearing schools, and two received a mixture of Deaf and 'mainstream' schooling. Of those educated in mixed environments, both identified as bicultural (Hearing/Deaf), while all other participants identified as Deaf regardless of schooling - including the participant who was mainstreamed, spoke English, and used a cochlear implant.

Though the demographic questionnaire contained 11 questions, their provided responses to some questions contradicted later focus group discussion. For example, two participants reported that their PCP was not hearing, despite later discussions revealing that only one participant had ever seen a non-hearing provider, and this participant reported their current provider as hearing. These data are presented in Appendix I. 


\section{Overarching Themes}

\section{The Trust Kite}

To assist in the visualization of data, the imagery of a triangular kite was implemented (see Appendix J). In the figure, the fabric sail represents the beliefs and experiences of the Deaf patients while the person holding the spool represents the healthcare provider. These two are separated, but connected, by a quadruple threaded line representing the overarching theme, communication and its four subthemes: (1) conversational, (2) behavioral, (3) visual, and (4) environmental communications. Finally, each of the four identified themes (a) sameness, (b) power dynamics, (c) professionalism, and (d) culture clash are separated into their respective lobes along the base of the triangular kite.

The elements of this design are not arbitrary. The physical separation and positioning depicted between the healthcare provider and the kite signifies both the difference in role and positions of power in patient-provider relationships. In this depiction, the provider is in the position of power, and only able to interact with the patient through communication. To fly the kite and build trust, the healthcare provider is responsible for getting the kite off the ground and maintaining its trajectory. Over time, 'gales' may threaten the kite's ability to fly, but effective communication will allow the kite to overcome these adversities, letting it sail to new heights.

\section{Communication}

For the participants, communication incorporates more than the words we say and the way we say them. Though words are important, analysis of the focus group and interview data revealed four central forms of communication, many with little relation to 
linguistic communication: a) conversational, b) behavioral, c) visual, and d) auxiliary. These forms of communication are distinct. However, communication often contains indivisible aggregates of more than one form. For example, bypassing the interpreter to write a note directly to a patient contains conversational and behavioral forms of communication. In addition, combinations of each form act as the means through which the primary themes were experienced by participants because communication is how we convey meaning and intent. Therefore, each interaction is an opportunity to support or refute established narratives about healthcare providers and hearing culture. The following quotation is one such example and was provided by one of the youngest members of the focus group:

I know that [Deaf people] can support one another. We're the same. We're peers. Versus somebody who's hearing... We're not necessarily peers. Maybe they don't have the same respect. It's not necessarily that I can't trust them, but I have to (pause) - it takes more for me to trust them. I have to teach that - I have to develop that trust. (Participant 7)

Conversational Communication. Conversational communication incorporates all of the words and signs used during direct communication between patients and providers. This includes 'indirect' communication through an interpreter. This subtheme includes how PCPs communicate "I'm more apt to trust that doctor because he directly communicated with me" (Participant 3); the words they choose, "When I see all them big words, I don't know what they mean" (Participant 6); and even whether they choose to communicate at all, "I want them to explain more, in detail" (Participant 8). Perhaps more than others, use of this communication form signals the 'reference point' of the provider, characterizing the lens through which the provider, as an individual, views Deaf culture. Like all forms of communication, this characterization informs the Deaf patient 
how - not whether - PCPs perceive equality, power, professionalism, and culture. For example, in response to a healthcare provider referring to a participant as "hearing impaired", the participant refuted the assertion by emphasizing the healthcare provider viewed his hearing status from the wrong perspective: 'No. I'm Deaf. You know, I'm fourth generation Deaf so I'm - I didn't lose anything” (Participant 10).

Behavioral Communication. Behavioral communication complements conversational communication. Both incorporate elements of how and whether healthcare providers communicate with patient who are Deaf, but behavioral communication focuses on body language, including eye gaze, which can convey meaning without the use of words. This form of communication typically displays a healthcare provider's comfort interacting with people who are Deaf:

Like your body language, your behavior, your facial expression. Like, you know. If they kind of freak out, like "How am I going to communicate?" It's almost like they're scared or timid. You know, and then they shift aside (participant imitates behavior) before they go in [thinking] exactly how they're going to show themselves and prove themselves to us. (Participant 2)

Visual Communication. Visual communication is perhaps the most abstract form of communication to hearing healthcare providers. Although hearing people use visual cues to make decisions, it is often not the first or primary sense through which they experience the world. In contrast, sight is often the primary (and only) sense used by Deaf participants during communication. This contrast makes Deaf people hyperaware (by comparison) of visual anomalies in facial expression, gesturing, and timing that might suggest a lack of acceptance. Several of these elements may also be classified as behavioral communication, but specific elements of sameness and professionalism are 
easily influenced by non-behavioral, visual cues. For example, some participants

expressed concern for a lack of cultural congruence based on a PCP's attire:

Facilitator A: So, you're saying if you see somebody... and they're wearing a hijab, that [person] is less trustworthy to you because you have cultural differences? Because [the hijab] is not a part of your culture?

Participant 5: Yes. That's right.

Participant 9: Yeah. That's why was talking about doctors who are Indian or Muslim. Sometimes, I just don't feel comfortable with them because their culture. I prefer a doctor who has a culture that's similar to mine. I'm more comfortable with them.

Environmental Communication. Environmental communication appears to play a more subtle role in signaling the intentions or reference point of a healthcare provider within the healthcare environment. Many times, this form of communication is the antithesis of visual communication, highlighting what is not seen or not present. One common form of environmental communication bemoaned by every participant in the sample, across several healthcare settings, was the exclusive use of aural forms of communication:

You know, in a doctor's office, when they yell your name. When they call your name, I don't hear anything. And they step out that door and they say your name and I misunderstood. Like who they're calling. And they don't know who I am, because I'm deaf. (Participant 2)

Whether these events led to a lack of interpreter use during the patient's visit was not discussed, but the lack of 'Deaf-friendly' communication prior to the scheduled visit (e.g., access to ASL) forced participants to ineffectively attempt other forms of communication such as lipreading and writing. In addition, reliance on these forms of less effective communication strategies forced many participants to miss communication which signaled a lack of consideration for their needs. 


\section{Major Themes}

Data analysis revealed four primary themes: (a) sameness, (b) power dynamics, (c) professionalism, and (d) culture clash. Three of these major themes contain subthemes, and each of these major themes contain strands of the overarching theme: communication.

\section{Theme 1: Sameness}

One recurring theme experienced by everyone in both focus group and interview was a lack of recognition regarding their personhood while receiving care. The degree to which the feeling of personhood was withheld differed for each participant and was contingent on the specifics surrounding the experience, but the general feeling was ubiquitous - inadequacy and insignificance when viewed by both healthcare providers and hearing society. Participants typically framed this experience in the affirmative, emphasizing that we should all be treated as equals, but deeper investigation revealed an intended emphasis on equity, not equality.

Mild experiences focused on feeling 'lesser than' able-bodied people:

Recently I had a slight back injury and my doctor was not available, so there was another physician that was able to see me, and the first questions she asked me was 'How did you become deaf?'. And, I'm like, 'I'm not even here about that. I'm here about my back.'... I'm like, 'Does she look at me as being a broken individual... or is she really genuinely curious because she knows how to communicate?'. (Participant 10)

Other participants focused on a lack of common courtesy or professional response to a request, signaling to them a lack of concern for, or dismissal of, the needs of the participant:

The interpreter has the appointment. Everything is worked out, and that's fine, and I leave, and then it's close to the appointment. I'm like, 'Okay, before I leave the doctor's appointment I say [to myself], "Make sure you call and set up an 
interpreter," and then I'll call and say, "you got that interpreter," and they're like "(exacerbated sigh)". So then, like, the interpreter is late showing up, and everybody else is being seen in front of me, and my time is valuable too, but I keep getting pushed back. So, like, you know. Two / Three days later I have to come back. ... It's a waste of my time. (Participant 4)

More extreme examples appear to manifest when healthcare providers blatantly ignored the participant. Some compare this lack of acknowledgment to being deemed subhuman.

You know, I just prefer them to be looking at me. That way, I can (pause). Like even though the interpreter's over here, and signing, and I look at the interpreter. The doctor's looking all over the place. I want the doctor to look at me and acknowledge me as a human. (Participant 2)

The desire to have PCPs look directly at the participant during a healthcare visit was shared by nearly every member of the focus group. However, only a few openly expressed their agreement with feeling subhuman by the healthcare provider's actions.

Further exploration of this comment during a member check revealed a story, unconfirmed by this author, that at one time hearing people thought ASL was first created to communicate with monkeys and later taught to people who are Deaf (presumably) because they lacked the capacity to communicate through other means. This allegory, true or not, provides insight into the perceptions belief systems within Deaf culture, including the general belief beliefs that hearing people equate signers to monkeys. It also shows how a lack of acknowledgment can be viewed as signaling something so perverse. For one participant, the desire for acknowledgment was so strong that he praised a healthcare provider for bypassing the interpreter to communicate directly with the participant, as follows:

When the doctor is finished, they come up to me. They're not talking. Just showing me a piece of paper that said "I'll be back in a moment. Okay?", and the interpreter didn't have to tell me. I was impressed with that because [the 
healthcare provider] felt comfortable enough to come to me - directly. (Participant 3)

When discussing this concept of sameness, the signs "EQUAL" and "SAMESAME" were used, but further analysis revealed that most of the time equity, not equality, was the desired result. For example, participants expressed the desire to be treated as equals with the same level of autonomy, respect, and personhood as healthcare providers, and insisted that they receive the same quality of treatment as hearing people. This suggests the inclusion of accommodations makes the Deaf patient feel equal in their autonomy and individuality. For example, participants expressed a desire to effectively: 1) communicate with a healthcare provider, requiring an interpreter, 2) be identified in a waiting room, requiring a visual - not auditory - cue, and 3) be informed about what is written in their medical chart, which requires the interpreter to interpret what the healthcare provider writes. These actions are not 'normal' in that most hearing patients do not receive this style of care (equality), but the end result is one in which deaf and hearing patients receive the same experience (equity).

\section{Theme 2: Power Dynamics}

The data revealed power as a major theme, with two subthemes: Powerlessness and empowerment. Each appear to exist as opposite sides of the same coin, experiencing less powerlessness when empowered and vice versa. In addition, the healthcare system empowers healthcare providers more than patients. Therefore, PCPs are best positioned to equalize the difference in power.

Powerlessness. Many degrees of powerlessness were expressed by participants, ranging from abdication of trust to general lack of control. However, abdication of trust was expressed by only two participants: One of the youngest and one of the oldest 
members of the group. The older member, recalling his youth said, "You know. I didn't know that person, but Mom trusted them growing up. So, you knew that person is trustworthy because someone else trusted them" (Participant 2). The younger member demonstrated, rather than articulating, his abdication. The best example came when the younger member was asked questions about the trustworthiness of the PCPs in a story he told about an acquaintance. When asked how the participant knew the PCP was trustworthy, the younger participant said, "I don't know... That's what my teacher told me" (Participant 4). Other examples of powerlessness were expressed as a lack of control.

Examples of general powerlessness include the lack in ASL interpreter availability with no way to influence the situation. The best example of this came from a highly coordinated healthcare experience involving the participant, individuals at their primary care office, a third-party healthcare practitioner, and an ASL interpreter. In this example, the participant was powerless in his attempt to receive the test he needed because of consistent problems booking an interpreter. For example:

I had an appointment. [The office] brought someone in for me. I knew them. They were going to be doing a test... I asked them where the interpreter was and they told me [the interpreter] was postponed. So, I was going to have to come back. ... I knew that man who would be coming back [to administer the test] week after week, but [the office] postponed [the appointment] again. So, then I had to come back at a later date. (Participant 7)

Other examples of general powerlessness highlight the imbalance between patients who have no choice but to adhere to PCPs, even if the patient feels the PCP is making lucrative healthcare decisions at the participant's expense:

I've seen with doctors, often they'll make a mistake and take advantage [of their patient], and they'll need you to come back again and change your medication again. I think it's related to finance. I think it's related to money. (Participant 6) 
Empowerment. Empowerment, not power, is the opposing force in patientprovider relationships. Examples of empowerment described by focus group participants include the ability to choose a healthcare provider with whom the patient felt comfortable and having access to general health information (often written in English), as follows:

My wife researched online to try to find the best doctor, and they referred us here. And, we were able to find a lot of different information and brochures about them to see that this is the top place, and we knew to come here. Based on the images and research that we found. And, that's why we picked that. Based on our research. (Participant 9)

Other times, power was explicitly given to the patient by the healthcare provider:

"I'm getting older and so I needed to have a colonoscopy. So, when I got close to the age I needed to have one, my doctor let me know, and we talked about it" (Participant 10). In this example, the PCP empowered the participant by informing the participant about preventative health screenings and sharing the responsibility to make decisions about the participant's health.

\section{Theme 3: Professionalism}

Discussions of professionalism were both explicit and implicit throughout both the focus group and the one-on-one interview. In every case, being professional (or perceived as having a high degree of professionalism) was associated with being trustworthy, as follows, "if you're going to a good professional doctor instead of a doctor - compared to a doctor who isn't professional, you know the professional one is trustworthy" (Participant 8). Further analysis revealed professionalism to be a multifaceted construct of its own. Several participants used the term differently, but most participants expressed their agreement with professionalism in terms of quality and duration of their healthcare experience. 
Participant 4: You know, like a surgeon, for surgery. I wouldn't trust a person that was, you know, one year into it. I would obviously want a person with more experience.

Participant 3: Exactly. You only want the professional doing surgery, you know, doing surgery on you.

Participant 4: [The surgeon] has a couple years of experience. I would prefer him over a person who's new.

\section{Participant 1: Always}

Other participants equated professionalism to several additional attributes. These include professional communication, “A professional physician. They’re good. I understand them" (Participant 8), confidentiality, "you're able to find another doctor you might be more comfortable with, whose more friendly, who's more confidential" (Participant 9), general appearance "and then, you're able to find another doctor you might be more comfortable with... who dresses nicer than a doctor who is maybe a slob, and who is a mess" (Participant 9), and having a license: "is 'professional' a behavior, or is 'professional' a license?" (Facilitator A); “a license” (Participant 8).

\section{Theme 4: Culture Clash}

A common pattern among focus group and interview grievances was revealed about cultural differences between Deaf culture and hearing culture predominant in healthcare. Some of these differences are rooted in general differences between hearing culture and Deaf culture. For example, using auditory cues instead of visual cues when identifying patients in waiting areas "You know, sometimes they're screaming. I'm like, 'I can't hear" (Participant 3), explaining procedures while actively preparing for the same procedure, “They turn off the lights, and I'm like, 'Nope, I need the lights on [to communicate]" (Participant 10), and not providing enough eye contact, "I just feel like 
they're ignoring me and looking at the computer the whole time" (Participant 4). In addition, large divides exist between healthcare cultures and Deaf cultures, including a clash between the task-oriented healthcare systems and relationship-oriented Deaf communities, and what constitutes patient-centered care.

Task-Oriented vs. Relationship-Oriented Cultures. Several participants expressed frustration with providers using terms like 'hard-of-hearing' or 'hearing impaired'. To the participants, use of these terms signaled a lack of cultural competence, a view of deafness from a hearing reference point, and by extension, a discriminatory perception of Deaf people. This sentiment was discussed by almost every participant during the focus group, but perhaps the best example of this frustration and its significance was demonstrated during the one-on-one interview. As one participant described:

When I first met my doctor and she said - she was going through my medical history reading from the form I filled out - and she said, "I see you're hearing impaired," and I was like, "No. I'm Deaf." You know, I'm fourth generation Deaf so I'm - I didn't lose anything. I was born deaf, and that really set me (pause) That really made me not trust her at first. You know, like deficit thinking like that is a problem. I want us to be equals. If I'm going to trust you, I want to know you see me as a person, and not a disability. So, I want you to ask me how I identify before you assume I lost my hearing. (Participant 10)

Several minutes later the participant's opinion of his PCP shifted, after she made concessions regarding her use of terminology, as follows:

With my doctor, after I corrected her and said 'I'm Deaf" she started using my language to identify me. Now, when I go into see her she asks about my hearing by saying, "Has anything changed with your hearing" and that's fine, because, although I'm not there for that, I know she sees me as a person and she's just asking to make sure there're no new issues we need to look at. (Participant 10)

There were many more quotations exemplifying the culture clash between the different cultural orientations by members of both the focus group and the one-on-one 
interview. In the previous quote, the participant expressed his initial frustration with a new PCP because, from his perspective, the PCP misidentified his cultural identity and perceived him as someone with less than 'ideal' hearing. Later, the participant expressed less concern regarding the PCPs continued inclusion of his hearing status in routine care visits because he "know[s] she sees me as a person". Additional information provided in these quotations suggested the PCP was performing a routine assessment, which includes asking direct questions about provided information to verify and contextualize the patient's health status in the most efficient way possible (task-oriented communication). The situation was only ameliorated after the PCP sufficiently accommodated the Deaf participant, building a relationship through which tasks can be completed (relationshiporiented communication).

This participant further solidified his view about the perspectives of PCPs towards Deaf people in a closing statement. This statement highlighted the participant's desire to include more Deaf cultural content in healthcare courses, but more importantly, it highlighted a basic assumption that the perceptions of healthcare providers towards people with 'imperfections' mirrors the deficit language used to describe these deviations from the statistical norm.

I think there is more that should be done, and can be done, to eliminate any negative perception of any Deaf and hard of hearing people, and central biases, any (pause) you know, negative perceptions of Deaf and hard of hearing individuals. That is what comes to mind for me is there needs to be that particular area of emphasis and I don't think there is an emphasis on the need to make sure that providers are culturally sensitive and can provide culturally sensitive care. (Participant 10)

Patient-Centered Care. Another culture clash between healthcare culture and Deaf culture relates to patient-centered care. In many ways, participants expressed a 
desire to be 'equals' or collaborators in their care, as follows: "If they're acting in a way that isn't culturally competent and not treating me as an equal partner in my care, that indicates to me that there might be an issue" (Participant 10). However, there appeared to be a clash regarding what a patient-provider partnership means. Some participants toggled between referring to healthcare providers as partners "My doctor and I decide what's best for me" (Participant 10) and employees, "Are they willing to do what it takes to make sure that my needs are completely met, completely satisfied, before I leave today? (Participant 10).

One participant also alluded to a partnership while acknowledging a separation in roles, as follows: "I want the doctor to be motivated to really tell me like all these different things [about my care]" (Participant 6). However, several examples depicted the role of the PCP in ways akin to personal accountants, hired for a specific purpose and required to fulfill that role in a specific way. For example, one participant spoke truthfully about the "legal obligations" of healthcare providers providing licensed ASL interpreters, but disapproved of how this service was provided, as described in the following quote:

When I go, thinking there will be an interpreter, but it's through VRI [Video Remote Interpreting services]. So, they were honest. They do have an interpreter, but it's not a real interpreter. They have access to an interpreter, but now I have to be willing to navigate the screen and communicate when (pause). You know, they're terrible. Yeah, they work, but they can't see or interpret what's going on, and they're hard to see. (Participant 10)

Another participant was emphatic that the participant always be accompanied by a licensed ASL interpreter when attending a PCP visit, even in the unusual situation where the PCP also happens to be a certified ASL interpreter, as follows: "If the doctor [says,] 'I know how to sign. Means you don't have to have an interpreter.' I'm like, 'No. 
Absolutely not! I need an interpreter there. Period" (Participant 3). PCPs with dual certifications as providers and licensed interpreters in ASL are rare, but this quote highlighted the difference in cultural perceptions surrounding primary care, suggesting providers lack the ability to effectively practice in a second language, by adding the following: "They're focused on separate responsibilities. One is focused on interpreting... The other is focused on medical information... So that their brains are able to focus [on] specific tasks at hand" (Participant 3). This sentiment was adamantly supported by three others, one of whom added the following: "[I'm] saying be focused on one thing. And then waiting, and then focusing on the - him as the patient, second" (Participant 4).

Finally, a different participant wanted to know everything being written in their health record to improve their ability to understand their discharge paperwork, "I want to know what the doctor's typing... I think that's very important... Just to make sure it makes sense in the computer, and [I'm not thinking], like, 'What's on the [discharge] paper" (Participant 1). This particular quote highlighted a lack of understanding by the participant regarding the use and purpose of charting, but moreover, these examples as a whole showed the general misunderstanding of the parameters and mechanisms involved in healthcare delivery, as well as what patient-centered care means to PCPs.

\section{Discussion}

\section{Similar Experiences}

Before discussing the specific themes identified in this study, it is important to comment on the similarity to which the participants of this study experienced healthcare settings when compared to other people who identify as culturally Deaf. For example, 
every participant described some level of anxiety, fear, or confusion with waiting rooms, a sentiment expressed by dozens of others (DeVinney \& Murphy, 2002; Iezzoni et al., 2004; Reeves \& Kokoruwe, 2005; Ubido et al., 2002). Barriers to care like poor coordination with ASL interpreting services were expressed (Iezzoni et al., 2004), and PCPs not using licensed interpreters when required were expressed (DeVinney \& Murphy, 2002; Reeves \& Kokoruwe, 2005). Participant complaints about the use of technical jargon (Ubido et al., 2002), and beliefs about the negative views purportedly held by PCPs towards people with deafness (Anderson et al., 2017b; DeVinney \& Murphy, 2002; Iezzoni et al., 2004) have also been expressed by others.

\section{The Themes}

Several of the themes identified in this manuscript were expressed by others. In particular, communication is by far the most frequently referenced concept in healthcare literature pertaining to people who are Deaf. Other themes, like sameness (Witko et al., 2017), power dynamics (Anderson et al., 2017b), professionalism (Cabral et al., 2013), and the culture clash between healthcare services and Deaf culture (Harmer, 1999) are also identified. However, few publications discuss these themes in the depth provided by this study or provide commentary on the expressed experiences in relation to trust.

\section{Communication}

Communication is by far the most ubiquitous aspect of the patient-provider relationship identified in this study. Communication in one form or another directly impacted all aspects of Deaf participants' perspectives about PCPs, mirroring the works of others. For example, within the healthcare literature, effective communication with people who are Deaf is discussed as an important part in decreasing feelings of fear and 
mistrust (Steinberg et al., 2006), instilling inclusion (Meador \& Zazove, 2005), and building trust (Cappel, 2009). However, the majority of publications referencing communication identify, but do not discuss, its significance. Likewise, many authors identify the importance of specific aspects of communication, like eye gaze (Lieu et al., 2007), without discussing its relationships to communication and trust.

Understanding the four forms of communication identified in this study are important when seeking to build trust in any patient-provider relationship involving people who are Deaf. Many authors provide commentary on conversational communication, but miss the opportunity to discuss behavioral, visual, and environmental forms of communication. Analysis of the data suggests that egregious conversational communication resulted in the most visceral responses from Deaf participants, but visual forms of communication (including examples that may also be classified as behavioral or environmental) were more prolific and essential for establishing and maintaining trust.

For example, using the term 'hearing impaired' while talking to a participant immediately threatened trust in the relationship and warranted a correction, but behavioral signs of discomfort were only met with general discomfort, despite its contribution to the degradation of trust.

\section{Sameness}

When seeking a PCP, participants in other studies identified the desire to work with someone with shared life experiences (Anderson et al., 2017b). Perhaps this is why participants in other studies reported a desire to seek health information from other Deaf people before seeking advice from hearing professionals (Cabral et al., 2013). However, the theme of sameness pertains to more than identity and experience. 
Sameness also refers to the feeling of being equal, though different, in the eyes of those in power. For example, one researcher reported that lack of recognition by healthcare providers elicited feelings similar to "not being human" (Witko et al., 2017), and others reported that "people who leave feeling as though they have been treated as a non-person may be reluctant to go back to the doctor or hospital" (Robins \& Mangan, 1999, p. 31). This experience is not unique to Deaf people or Deaf culture. Within the disability literature - to which the Deaf do not align their identity - feelings of being treated 'less than' able-bodied people through exclusionary practices often ingrained social norms (Valente \& Danforth, 2016). These stories, and others, have promoted many to question the use of labels, like disabled, in nursing (Alex \& Whitty-Rogers, 2012) and other fields (Akhtar \& Jaswal, 2013). However, labels may be paradoxical. On one hand labels perpetuate standards that contribute to society's continued implicit biases (VanPuymbrouck et al., 2020), but on the other hand, labels can be a source of power to those who incorporate a label into their identify (Mogensen \& Mason, 2015; Hansen et al., 2014).

\section{Power Dynamics}

During any given appointment, care decisions are influenced - if not entirely controlled - by people other than the patient (Collyer et al., 2017). This power dynamic is further exacerbated when patients and providers speak different languages, transferring power to interpreters (Baker-Shenk, 1992), leading some scholars to report heightened concern regarding learned helplessness among patients who are Deaf (Harmer, 1999). However, this was not reflected in the sample. Only one participant discussed their healthcare experiences with any hint of helplessness, suggesting a possible change in the 
way modern patients who are Deaf perceive their role in healthcare settings when compared to the views expressed about similar patients near the turn of the century.

One stark difference between the experiences expressed by the participants in this study and the experiences of others in the literature is a lack of physical harm resulting from poor or incomplete communication. In this study, participants were instructed to avoid discussing protected health information, which may have influenced the lack of reported harm experienced by the participants. In contrast, several studies have presented information regarding harmful events caused by poor communication or naïve acceptance of care. One such example includes the wrongful administration of medication (Reeves \& Kokoruwe, 2005).

\section{Professionalism}

On the whole, being professional is not something readily discussed in Deaffocused health literature. However, aspects of professionalism are discussed. Among them, confidentiality is more frequently cited, but most comments about confidentiality focus on interpreters (Anderson et al., 2017b; Crowe, 2017; Mprah, 2013), other Deaf people (Cabral et al., 2013; Cabral et al., 2014; Harmer, 1999), and researchers (Meador \& Zazove, 2005) rather than PCPs. An interesting observation identified in this study is the existence of 'status' trust: the idea that an individual's status makes them more (or less) trustworthy (Lount \& Pettit, 2012). In this study, several participants reported that PCPs with 'professionalism' were more trustworthy, and that PCPs had more professionalism simply by virtue of their license. Therefore, PCPs were perceived as more trustworthy by virtue of their license. Likewise, experience acted as a form of status, instilling higher degrees of trust with each year of service. 


\section{Culture Clash}

Any generalization about Deaf people and Deaf culture may be dated or anecdotal (Miller, 2010), but a comparison of results of this study and results from other studies highlighted fairly consistent cultural contentions. Harmer (1999) discussed the cultural tendency for Deaf people to tell stories rather than provide parsimonious anecdotes to questions, highlighting a key difference in relationship-oriented Deaf culture and taskoriented healthcare culture. Meador and Zozoke (2005) described this communication difference as English 'working up' to the main point and ASL 'winding down'. Likewise, ASL is blunt, despite its use of storytelling (Moore \& Levitan, 2011), while many English speakers use passive forms of communication. For example, glancing at the clock to signal the end of a session or speaking figuratively (rather than literally) about disappointing results (Kaplan, 1996). These forms of communication can be confusing if not entirely ineffective to ASL users (Kaplan, 1996).

The desire to increase cultural competence among PCPs that was expressed in both the focus group and the one-on-one interview was also expressed in the literature (Ferguson-Coleman et al., 2014; Mprah, 2013), including the use of warm and friendly environments (Anderson et al., 2017a) with good lighting (DeVinney \& Murphy, 2002). However, no publications were found that identified the desire of Deaf people to learn more about healthcare culture. In this study, several participants discussed their desire to learn more about healthcare knowledge (e.g., anatomy, physiology, and pharmacology), but no participants expressed a desire to learn more about how healthcare works. In their defense, this study did not actively investigate the desire of participants to understand 
healthcare culture, but a deeper understanding might alleviate confusion and dissatisfaction by setting more realistic expectations about healthcare systems.

Some participants from this study expressed a desire to learn more about healthcare culture, including the participant who expressed speculation regarding his need for multiple visits without acknowledging the use of medication titration. This participant (Participant 6), as well as the participant who disapproved of Video Remote Interpreting services (Participant 10), may not have considered the negative financial balance accrued by PCPs when using live interpreters. Likewise, they may not have considered the standard of care provided to other non-English speaking patients including poor or entirely lacking visibility during a healthcare visit interpretation (e.g., interpreter phones). The participant who insisted on having a PCP and an interpreter at all visits (Participant 3), and those who sympathized with this request, may not have considered how and why PCPs simultaneously discuss and record patient information in any language they hold fluency. Finally, the participant who wanted to know everything that was written in his health record (Participant 1) may not have considered the purpose of notes and how they differ from discharge instructions. These examples did not suggest healthcare systems cannot or should not change to accommodate the needs of others. Instead, these examples suggested full accommodation for the preferences of Deaf people can place a heavy burden on healthcare systems. For example, the desire to never book two Deaf patients "back-to-back" for fear that their confidentiality will be breached if one of them sees the other's car (Anderson et al., 2017a).

Of course, not all Deaf people share the expressed desires of a few. Most notably, several researchers found a preference by Deaf people to directly interact with an ASL- 
fluent healthcare provider rather than use an interpreter during healthcare visits (Anderson et al., 2017a; Cabral et al., 2013; Feldman \& Gum, 2007; Steinberg et al., 2002). These findings differ from the findings of this study in which several participants expressed a desire to have an ASL interpreter in addition to a PCP rather than rely directly on an ASL-fluent healthcare provider.

Something not discussed in this study, but frequently reported by other scholars is PCPs covering their mouths while talking (Reeves \& Kokoruwe, 2005; Robins \& Mangan, 1999). Many PCPs may assume this only applies to the use of masks, but facial hair was the most frequent offender (Iezzoni et al., 2004; Kaplan, 1996; Wright, 1993). In addition, looking away or bowing their heads was equally disastrous for those who relied on lip reading (Iezzoni et al., 2004; Reeves \& Kokoruwe, 2005). This may explain why three participants preferred for their PCPs to look directly at them when talking, finish talking, and then look away to complete a task. As an extension, facial expressions are used to convey meaning in ASL (Kaplan, 1996). Therefore, the expressionless delivery with which PCPs are trained to provide healthcare information is not helpful, and "bad facial expressions" are deemed inappropriate (Anderson et al., 2017b).

\section{Integration with the PMT}

\section{Factors of Perceived Trustworthiness}

The PMT framework identifies three perceived attributes responsible for determining trustworthiness: Ability, integrity, and benevolence (Mayer et al., 1995). During the focus group and one-on-one interviews, participants uniformly identified the importance of ability in determining the trustworthiness of a PCP. However, discussions 
about the importance of integrity and benevolence were initially refuted, though they were later revealed as important through analysis of the stories about their experiences.

One participant suggested the attribute of ability included two separate skill sets: hard skills and soft skills. Hard skills were those needed to "do your job... [including] diagnose and prescribe medicines". Soft skills were those involving communication and general interaction. This dichotomy was not echoed by others, though all participants articulated the importance of hard skills and soft skills as this participant described. A review of Mayer et al. (1995) and Schoorman et al. (2007) suggests hard skills are ability attributes, as defined by the PMT. These include all physical and mental aspects of completing a desired task, like providing adequate patient care. Soft skills, as defined by the participant, do not address these aspects of care, and therefore, are best represented by either integrity or benevolence depending on the specific skill in question.

In this study, the four themes of sameness, power dynamics, professionalism, and culture clash predominately align with the attributes of integrity and benevolence, although exceptions exist. For example, comments about confidentiality and maintaining a professional license suggest the theme of professionalism inhabits the attribute of ability, but the professionalism element of attire aligns with integrity, and communication aligns with both integrity and benevolence. Mayer et al. (1995) distinguished between integrity and benevolence by emphasizing the importance of time in perceiving benevolence. When individuals meet, previous knowledge about a particular person mixes with first impressions to place a strong importance on the attribute of integrity. As time progresses, and individuals are able to observe one another, perceptions about the intentions of others toward the person doing the trusting become more influential. In this 
way, perceptions of integrity are strongly influenced by first impressions while perceptions of benevolence are strongly influenced by continued engagement over time.

In the identified themes, examples representing the attributes of integrity and benevolence were expressed for each major theme. For example, use of deficit language like hard-of-hearing or hearing impaired immediately triggered a lack of trust in participants by signaling a negative perception about people who are Deaf. This example spans the themes of sameness and culture clash, but the alignment of this example to the attributes of integrity and benevolence depend on when the terms were used. If a participant was called 'hearing impaired' at their first meeting, this example would influence perceptions of integrity. If a participant was called 'hearing impaired' years after an initial interaction, this experience would influence perceptions of benevolence. During the focus group discussion and the one-on-one interview, both versions of this scenario were described.

\section{Trust and the Trust Kite}

Revisiting the analogy of the trust kite, a previously mentioned element should be further discussed to integrate the factors of perceived trustworthiness (i.e., ability, integrity, and benevolence) with the themes identified in this study (i.e., sameness, power dynamics, professionalism, and culture clash). As previously stated, the kite's fabric (i.e., 'sail') represents the beliefs and experiences of the participants, but like real kites, sections of the sail can be differently colored to represent the proportion of the trust experience influenced by the factors of perceived trustworthiness (see Appendix K). From an aerial view, the top third of the triangular kite would be one color, representing the ability attribute. Its domain does not incorporate the themes identified in this study, 
and therefore is physically separated from the base of the triangle. The remaining two thirds will change colors over time as the identified themes transition from strongly influencing perceptions of integrity to perceptions of benevolence. In addition, data from this study would suggest trust kites for people who are Deaf might have larger sections dedicated to integrity than benevolence, even late in a relationship, because Deaf culture places a higher value on adherence to cultural values (a condition of integrity) than other cultures.

The Graph. Using a standard scatter plot with trustworthiness on the Y-axis and time on the $\mathrm{X}$-axis, one could visualize the trajectory of a trust kite away from the $\mathrm{Y}$-axis as the trustworthiness of a PCP improves or declines from the perspective of a Deaf patient. Using concepts from the PMT (Mayer et al., 1995), we can discern that the Yintercept would represent the participant's unadulterated propensity to trust ('t 0 '). The first point after the $\mathrm{Y}$-intercept, and every subsequent point before the time of the first PCP interaction (' $t 1$ ') would represent the change in trustworthiness based on information indirectly gained about the PCP from other people and similar life experiences. At this stage, the kite's sail is heavily proportioned to include integrity, potentially leading to drastic changes with small amounts of information. After the initial interaction, each subsequent point (' $\mathrm{t}^{\mathrm{n}}$ ') would represent an additional change in trustworthiness as new information about the PCP is gained. During this time, the sail of the kite would change to incorporate more shades of benevolence and fewer shades of integrity, with the hope that eventually the trustworthiness of the PCP will sail to its maximum height. 
Additional factors could be represented on this scatter plot. Keeping with the conceptualizations of trust used to construct the PMT (Schoorman et al., 2007), the top of the $\mathrm{Y}$-axis would represent total and complete trustworthiness and the $\mathrm{X}$-intercept would represent a total and complete lack of trustworthiness. As such, the propensity to trust others may create unexpected, horizontal asymptotes, preventing a PCP from obtaining maximum trustworthiness. Likewise, a unidirectional, horizontal asymptote may exist for any PCP whose trustworthiness falls below a certain level, representing a point at which a patient is unwilling or unable to build trust.

\section{Limitations}

This study contains several limitations. First, a convenience sample was used to identify ten participants. Though these participants articulated many of the same sentiments found in the literature, data from this study cannot be generalized to all Deaf populations. Likewise, though the sample was diverse in terms of the represented age categories, race, and education, the sample was fairly homogenous regarding their Deaf identity and onset of deafness. Therefore, these data may not be indicative of people with late onset deafness and hearing identities, people who use cochlear implants and effectively communicate in English, or people living in different regions of the country or the world. In addition, using transcripts created from the spoken interpretations of the focus group creates additional opportunities for data to be lost. Though member checks were employed to minimize data loss or general misrepresentation, future studies should implement a data capture and data analysis plan that minimizes translation and interpretation of data. 


\section{Conclusion}

This study focused on trust and trustworthiness in the patient-provider relationship from the perspectives of participants who identify as culturally Deaf. Findings revealed four major themes (i.e., sameness, power dynamics, professionalism, and cultural competence) and one overarching theme (i.e., communication) with four subthemes (i.e., conversational, behavioral, visual, and environmental communication). Discussions about the healthcare experiences of patients with Deaf identities reported in previous studies expressed similar experiences, with some researchers identifying similar themes. However, the identification of communication subthemes is poorly represented in the healthcare literature.

Among the most important findings is that trust within the patient-provider relationship appears to mirror the Proposed Model of Trust developed by Mayer et al. (1995) with few alterations. In addition, the continued divide between Deaf culture and healthcare culture stems from a lack of understanding, possibly due to a lack in identifying the existence of the other culture. As such, PCPs can and should do more to accommodate the needs of people who are culturally or functionally Deaf.

Focusing on the four subthemes of communication, PCPs should be more cognizant of their behavioral, visual, and environmental communication during visits. This includes approaching potentially challenging situations with aplomb and providing enough space and light to ensure effective communication. Most importantly, it is important for the PCP to remember that the conversation has not ended just because no one is talking. Results from this study indicated that behavioral, visual, and environmental forms of communication most frequently apply in situations where 
conversational communication is not actively happening. Therefore, changes in facial expressions and body mechanics are likely to be noticed and if uncontrolled, will convey unintended messages. The 'hearing' equivalent, might be breathing without controlling your vocal cords. You may not mean to communicate through sound, but that doesn't mean the noises you make are not contributing to the underlying message being communicated.

When PCPs are aware of these additional forms of communication they can, and should, use them to their advantage. They should use affirmative forms of visual displays like head nodding when in agreement or when reaching a mutual understanding, puzzlement when thinking, joy when delivering universally 'good' news, and sadness when delivering 'bad' news. They should use open body language to indicate receptiveness, confidence, and a general degree of professionalism. Finally, making small changes to the design and workflow of the PCP office allows the PCP to communicate to their patients through the environment. Using visual cues like signs or physically retrieving patients from waiting rooms can signal a lot about the integrity of the PCP and the office staff.

Addressing the major themes of trust, PCPs should strive to treat all patients the same (equality), while assuring all patients get the autonomy and respect they deserve (equity). To achieve equality while maintaining equitable treatment, PCPs should seek to adopt universally affirming practices like asking everyone how they identify or prefer to communicate before making assumptions. PCPs should also strive to empower their patients by providing them with enough knowledge to make health decisions and sharing the responsibility of their care. Finally, PCPs should remember that healthcare has a 
culture, often unseen by others. For most hearing people, knowledge regarding the language, beliefs and norms of healthcare culture is gained passively through sound. This concept is often referred to as 'incidental learning'. However, people who do not communicate via sound often lack this form of incidental learning, making it harder to learn the cultural nuances of hearing cultures. Therefore, PCPs should strive to be good hosts by openly discussing expectations and cultural norms, even if they believe 'everyone knows it'. Likewise, all patients should be encouraged to ask questions about routine occurrences and behaviors using their preferred communication format.

According to the results of this study, making these adjustments should improve trust within the patient-provider relationship. Furthermore, improving trust may create opportunities to discuss differences in cultural norms and help patients and providers set more realistic expectations regarding mutual interactions by changing the way we interact with one another. After all, when we dare to see the world through the lens of another, we learn more about ourselves than if we never looked. 


\section{CHAPTER V}

\section{SYNTHESIS AND CONCLUSIONS}

The purpose of this dissertation was to: 1) identify the extent to which trust and trustworthiness were discussed in the nursing and allied health literature as they pertain to patient-provider relationships among people who identify as culturally Deaf, and 2) explore the trust in Primary Care Providers (PCPs) from the perspective of people who are culturally Deaf. In addition, this dissertation includes a chapter focusing on the challenges met while conducting cross-cultural research in American Sign Language (ASL) and using experienced licensed ASL interpreters from the perspectives of an ASL conversationally fluent, hearing researcher. This chapter synthesizes the research findings from the scoping review conducted on trust and trustworthiness in the Cumulative Index of Nursing and Allied Health Literature (CINAHL) database, and the qualitative analysis conducted on data collected from a focus group discussion and an independent one-onone interview about patient-provider trust. Finally, implications for policy, nursing practice, nursing education, and research are discussed.

\section{Synthesis}

\section{Introduction}

Interest in trust as a social phenomenon began in the late 1950 s and early 1960 s as a means of investigating conflict resolution through the work of the American social psychologist Deutsch $(1958 ; 1960)$. These early works primarily focused on theoretical aspects of trust that remained relatively unchallenged until the late 1970s during which 
theoretical debates on trust furthered its conceptualization into the mid 1980s. During this flurry of activity, empirical work addressing trust appeared in the healthcare literature when Caterinicchio (1979) tested the relationships among levels of trust (i.e., trustworthiness) in PCPs and several patient attributes including trust-taking behaviors (e.g., tolerance for treatment pain intensity) and health outcomes (e.g., perceived health gain). For over a decade, the work of Caterinicchio was the prominent source for understanding trust in healthcare settings, as his transition from the theoretical discussion to the practical application of trust set a precedent within the healthcare literature. From here, a surge of interest surrounding trust within patient-provider relationships began, focusing on practical application. This trend started in the early 1990s with the work of Anderson and Dedrick (1990) and continues today (Müller et al., 2014).

Building trust between patients and their providers is important because high levels of trust are positively correlated with several beneficial healthcare behaviors (Thom et al., 1999; Russell, 2005; Caterinichhio, 1979). As such, instilling trust can improve the health of patients and studying trust enables PCPs to better understand the ways in which trust can be instilled. However, two prominent gaps exist in the healthcare literature related to patient-provider trust. First, a lack of conceptual agreement between scholars has created a plethora of instruments measuring trust in different ways (Ozawa \& Sripad, 2013). Second, several studies identify the use of specific trust instruments among explicit cultural groups, and though almost all provide demographic information about their sample, a review of this literature revealed a lack of instrument application among people who identify as Deaf. Therefore, little evidence exists to support the use of any particular instrument to measure trust in Deaf populations. 
Studies investigating trust in general populations traditionally use unidimensional and interpersonal instruments like the Trust in Physicians Scale (Anderson \& Dedrick, 1990) and the Wake Forest Physician Trust Scale (Hall et al., 2002). However, some scholars use multidimensional instruments, like the Trust Scale for the Patient-Physician Dyad (Leisen \& Hyman, 2001) or instruments designed to measure trust towards healthcare systems, like the Public Trust in Dutch Health Care Scale (Straten et al., 2002). Moreover, debate regarding the relationships between trust and its antithesis, mistrust, has created a divide with the majority of scholars measuring mistrust as an independent construct, rather than the absence of trust, in minority populations (Ozawa \& Sripad, 2013). This lack of congruence creates a lack of cohesion within the healthcarerelated trust literature, making it difficult to determine which instrument to use with previously understudied populations.

To understand the extent to which these conceptualizations of patient-provider trust are established in the healthcare literature among people who identify as Deaf, a scoping review was conducted within the nursing and allied health literature. Data from this review was then used to inform a subsequent study to investigate the perspectives of trust and trustworthiness towards PCPs in individuals who identify as culturally Deaf. By reviewing established literature and investigating the gaps in this literature, researchers can better ensure trust-related data are collected, analyzed and disseminated in culturally congruent ways.

\section{Methods}

Prior to developing and conducting the qualitative portion of this dissertation, a review of healthcare-related, patient-provider trust literature was conducted with a focus 
on its implication for people who identify as culturally Deaf. This scoping review was originally conducted in the summer of 2018, with additional reviews conducted in the Spring of 2020. The scoping review was conducted by a single investigator using the Preferred Reporting Items for Systematic Reviews and Meta-Analyses extension for Scoping Reviews (Tricco et al., 2018) and the work of Arksey and O’Malley (2005). Data gathered from the scoping review was used to identify a gap in the literature regarding the conceptualization of trust in patient-provider relationships from the perspective of people who identify as culturally Deaf. The primary data collection component of this dissertation addressed this gap by asking participants who are Deaf about their perspectives of the patient-provider relationship. The Proposed Model of Trust (PMT), as described by Mayer et al. (1995), was used as a theoretical framework for this study. Using the PMT, questions were generated to address the key dimensions of trustworthiness and general perspectives about PCPs.

\section{Results}

\section{Results from the Scoping Review}

Few articles addressing the experiences of participants who identified as Deaf directly discuss trust and its derivatives (e.g., mistrust). Instead, several articles discussed the impact and importance to trust-related concepts (e.g., communication) in lieu of an open discussion about trust itself. In addition, several articles identified trust as a

problematic, if not volatile, construct in the patient-provider relationship from the perspective of people with Deaf identities. However, these examples of trust rarely received more than a cursory mention in the literature and several of these claims lacked adequate supporting citations. 
Communication, comfort, confidentiality, and respect are among the most frequent trust-related concepts contributing to the understanding of patient-provider relationships from the perspective of individuals who identify as Deaf. Of the 93 citations reviewed, 51 articles identified or discussed at least one trust-related concept, 42 included communication, while 21 included cultural competence, 19 included comfort, and 19 included respect. As a comparison, 11 articles discussed trust in a substantive manner, and 28 discussed at least one trust-related concepts in a substantive manner.

\section{Results from Qualitative Study}

Results from the focus group and independent interviews revealed four major themes and one overarching theme. Communication, the overarching theme, contained four subthemes: conversational, behavioral, visual, and environmental communication. Each form of communication was clearly demarcated from the other though several examples of the overarching theme contained a mixture of communication forms. Likewise, the four major themes (i.e., sameness, power dynamics, professionalism, and culture clash) existed independent of the others with several experiences revealing more than one theme at a time.

In addition, results from the analysis indicate that trust, as it is described in the PMT, aligns with the conceptualization of trust as it was discussed by Deaf participants. Two of the factors of perceived trustworthiness (i.e., integrity, and benevolence) subsumed the four major themes when participants contemplated the degree to which a PCP was trusted. The third factor of perceived trustworthiness (i.e., ability) existed independent of the major themes. Finally, the propensity to trust others, a moderating concept within the PMT, was identified within each of the major themes. 


\section{Integration of Results}

Trust as it is perceived by people with Deaf identities was rarely discussed in the nursing and allied health literature. However, several concepts akin to trust were discussed. Many of these concepts were explicitly identified by study participants recalling their experiences with healthcare providers and in healthcare settings. A comparison of the results reported by others and the results of this dissertation suggested trust is an integral part of the patient-provider relationship from the perspective of people who identify as Deaf. In addition, several of the concepts discussed and the quotations provided by other articles align with the themes identified in the qualitative portion of this dissertation. However, these themes were not the primary focus of these articles. Instead, these articles primarily focused on describing the experiences of participants who are Deaf by outlining barriers to care rather than connecting these experiences and barriers to larger themes within healthcare literature.

\section{Theoretical Frameworks in Deaf-Focused Research}

One possible reason for this lack of 'connection' is the infrequent use of theoretical frameworks to underpin study designs. For example, Steinberg et al. (2002) identified several themes akin to trust including understanding, communication, and ability but did not report the use of a theoretical framework to investigate these experiences. As such, this publication acted more as a statement piece about the experiences of Deaf people in healthcare settings than a comment on any one particular aspect of care. Steinberg et al. (2002) included several rich and important examples including problems with access to care, communication barriers, and use of negative imagery in the translation of medical vocabulary. However, the publication did little to 
integrate these findings into care delivery models or validate structural issues identified by researchers investigating other marginalized populations. Steinberg et al. (2002) did raise concerns about healthcare delivery, highlighted flaws in healthcare systems, and made recommendations for PCPs based on participant comments and legal precedent. However, without a framework or deeper discussions about the position of their findings within larger contexts, it is challenging to integrate these findings into larger concepts. In comparison, other articles that briefly provided substantive examples of trust also lacked a theoretical underpinning. The secondary analysis by Anderson et al. (2017a) successfully expanded the themes identified in a previous publication. During the first analysis, Anderson et al. (2017b) identified and discussed several of the same trustrelated concepts identified and discussed by Steinberg et al. (2002). However, in their later publication, Anderson et al. (2017a) repurposed their findings to make claims about trust-related concepts in research. Though the second publication could benefit from a theoretical underpinning, the repurposing of data to make broader claims about a nonDeaf specific concept (i.e., research) is an appropriate step forward.

Without doubt, several of the experiences discussed in the literature reviewed for this study were unique to people who identify as Deaf. Many of these experiences, including anxiety related to audible cues in waiting rooms and problems with finding physical interpreters, were identified during data collection and data analysis. However, the aforementioned trend of failing to provide contextual commentary about these experiences contradicts the underlying desire for equity discussed in Chapter IV. 


\section{Equity of Data Dissemination}

One of the major themes of the qualitative portion of this dissertation was 'sameness'. This theme held several subthemes, most notably the desire to be perceived as an equal and not 'lesser than' a person who hears. However, further analysis revealed that participants were more concerned with equitable treatment than equal treatment. Though the results reported in Chapter IV directly discuss sameness in the context of healthcare experiences, assimilation with the findings from the scoping review indicate a lack of equitable use of data.

Review of the literature showed that the majority of scholars interested in the experiences of Deaf people read (based on reference lists) and publish (based on journal of publication) in exclusive 'Deaf' journals. This practice isolates the knowledge generated by packaging and disseminating the content to a same niche community responsible for generating the content, creating an incestuous research environment. As such, it is easy for scholars from other disciplines and scholars not specifically interested in Deaf experiences to unknowingly ignore research about Deaf culture simply because assess to this body of knowledge requires addition awareness and effort. By comparison, scholars publishing on the experiences of people from other cultural groups are readily available in non-culturally specific journals.

One of the primary reasons Deaf scholars publish in Deaf journals is because Deaf scholars write about Deaf experiences. This process is not inherently flawed, but without fully integrating other (more prominent) topics in the research design and dissemination strategy, few non-culturally specific journals will want to publish the findings. On one hand, publishing articles specifically dedicated to the experiences of 
Deaf individuals is an effective way of maintaining equality. This is achieved by countering the overwhelming narratives of hearing experiences in research (Barnett \& Franks, 1999). However, on the other hand, not discussing the findings in the greater context of research endeavors makes the findings less enticing to non-niche scholars. As an extension, fewer journals may publish the findings, fewer scholars will access the findings, and fewer authors will reference the findings. In this way, the published findings will not be used equitably because equitable dissemination is achieved by contextualizing findings in ways that promote the inclusion of these findings in the greatest number of possible sources.

\section{Implications}

\section{Research Implications}

Trust is a heavily researched, and frequently discussed, concept in healthcare literature (Ozawa \& Sripad, 2013; Müller et al., 2014). Though this dissertation specifically focused on trust and the trustworthiness of PCPs, articles discussing trustworthiness among other healthcare providers are equally prevalent (Rørtveit et al., 2015). Yet, despite the ubiquity with which the term trust is applied, review of these systematic reviews did not yield a single study dedicated to perspectives of trust by people who are Deaf. Though it is possible the authors neglected to include a citation or that researchers neglected to provide the hearing status of their participants when providing their demographic data, the scoping review of the nursing and allied health literature provided in Chapter II only yielded 11 studies identifying the term 'trust' in conjunction with 'deaf' (duplicates and derivatives of trust not included). Of these, five were identified because the article referenced 'trust', as a financial organization, and four 
provided a cursory mention, leaving two articles with enough discussion to be identified as substantive. Likewise, of the 1912 citations identified using trust-related terminology, the majority $(n=461)$ focused on cochlear implants and discussed deafness from a pathological point of view. This lack of cultural representation of Deaf people in studies surrounding prominent healthcare issues and over pathologized body of literature of those included indicates the need for more culturally competent and inclusive research practices among healthcare researchers. Though several additional challenges must be overcome when conducting cross-cultural research, creating ASL-friendly content and incorporating people who identify as Deaf will enhance knowledge. Chapter III identifies several challenges that can be faced by researchers wanting to conduct cross-cultural research with Deaf populations, but perhaps the most important implication of these findings is for researchers to work towards establishing a network of individuals capable of conducting and contributing to nursing research in ASL.

\section{Practice Implications}

This dissertation focused on healthcare experiences of Deaf people with PCPs. As such, several of the implications stemming from the topics discussed and the themes identified in the qualitative portion of this dissertation are recommendations for the care and the care experience provided by PCP offices.

According to the PMT establishing trustworthiness requires a combination of ability, integrity, and benevolence (Mayer et al., 1995). PCPs must establish that they have the physical and mental capacity to accomplish the task at hand. They must demonstrate an adherence to a set of principles and cultural values the patient finds acceptable, and they must demonstrate a positive attitude towards the patient in such a 
way that the patient feels the PCP is motivated to act in the patient's best interest. This dissertation revealed that participants overwhelmingly believed PCPs possess the physical skill and mental acumen to diagnose and treat disease, but frequently lacked the tact to establish an acceptable set of cultural values. People who identify as Deaf are acutely aware of the historic and continued oppression of 'differently-abled' bodies. As such, many report a standing level of distrust toward healthcare providers.

One way to build trust is by providing culturally competent care before the PCP sees a patient who is Deaf. Examples include creating a warm and welcoming environment that accommodates their communication needs by using visual, instead of auditory, cues when interaction with patients. For example, flagging a patient's chart to indicate the need for the patient to be retrieved from the waiting room. An alternative opinion might include a 'buzzer' or text to indicate simple patient instruction, like 'Come to the door, please'. Likewise, ensuring interpreter services are available as soon as the patient arrives and that anticipatory information, like an estimated wait time, is provided upon arrival.

During visits, PCPs should remember that behavioral and visual communication does not stop as soon as people stop talking. Therefore, it is important to remember to actively use facial expressions and body language to indicate understanding or disagreement. Likewise, decoupling instructions from testing by first providing patients with instructions, and then giving the patient enough time to digest the information before proceeding with a procedure, may increase the quality and quantity of communication during the visit. 


\section{Education Implications}

Several participants commented on the need to increase cultural competence among healthcare providers. One way to do this is to increase awareness before PCPs graduate from their accrediting institutions. The Commission on Collegiate Nursing Education requires all nursing graduate to receive some form of diversity training; however, specifics associated with this requirement are not provided. As such, educational institutions need to take the initiative to incorporate education opportunities for students to learn about Deaf culture. This might include attending Deaf Events or partnering with local Deaf organizations to create healthcare themed educational material. These activities will not only provide additional opportunities for students to engage with course material, but it will create opportunities to make connections between Deaf and healthcare communities.

\section{Policy Implications}

The Americans with Disabilities Act (1990) requires PCPs to provide reasonable accommodations to patients who need accommodations. This includes arranging licensed interpreters for people who do not speak English and creating accessible environments for people with physical disabilities. However, though legal requirements are provided, little guidance appears to be given regarding the specifics of how these are to be achieved. For example, review of healthcare literature about the experience of people who are Deaf revealed an ongoing lack of accommodation for their needs in waiting rooms, a sentiment echoed in the qualitative portion of this dissertation. Though formal legislation regarding how accommodations should be provided in primary care settings may be an overreach of federal legislation, further guidance may be required. As such, 
professional organizations including the American Medical Association, the American Association of Nurse Practitioners, the American Nurses Association, and other allied health agencies like the American Dental Association should consider working with national agencies dedicated to the health and well-being of culturally Deaf people, like the National Association of the Deaf, to provide guidance in the form of policy statements. Similarly, regulatory bodies should include Deafness among their required diversity training. This could include degree confirmation coursework or revolving continued educational credits. Such actions would provide more visibility to this marginalized population and solidarity regarding the importance of including all people in care delivery models.

\section{Summary}

Trust remains an integral part of the patient-provider relationship. Instruments used to measure patient-provider relationships continue to be used and new variants continue to be developed. Yet few researchers conduct this research with members of Deaf communities. Likewise, researchers engaged with members of Deaf communities frequently fail to fully align their research questions with broader research trends. Researchers on both sides should continue their programs of research but should try harder to enrich their samples by including member of Deaf communities or diversify their foci to incorporate prominent healthcare themes. By doing so, researchers may continue to build on established literature in their respective fields while broadening the impact of their findings. Likewise, pooling available data to conduct secondary data analyses by comparing data across different studies may permit researchers the opportunity to publish in their respective fields, on the topics they find most pressing, 
while enticing scholars to investigate what the literature on deafness and Deaf people has to offer. 


\section{REFERENCES}

Agency for Healthcare Research and Quality. (2009). AHRQ activities using communitybased participatory research to address health care disparities. AHRQ. https://www.ahrq.gov/sites/default/files/publications/files/cbprbrief.pdf

Agha, A. (2007). Language and social relations. Cambridge: Cambridge University Press.

Alex, M., \& Whitty-Rogers, J. (2012). Time to disable the labels that disable: The power of words in nursing and health care with women, children, and families. Advances in Nursing Science, 35(2), 113-126.

Akhtar, N., \& Jaswal, V. K. (2013). Deficit or difference? Interpreting diverse developmental paths: An introduction to the special section. Developmental Psychology, 49(1), 1-3.

Altice, F. L., Mostashari, F., \& Friedland, G. H. (2001). Trust and the acceptance of and adherence to antiretroviral therapy. Journal of Acquired Immune Deficiency Syndromes, 28(1), 47-58. doi:10.1097/00042560-200109010-00008

American Annals of the Deaf. (2020). Unofficial title 'American Annals of the Deaf homepage'. Gallaudet University Press. http://gupress.gallaudet.edu/annals/

Americans With Disabilities Act of 1990, Pub. L. No. 101-336, 104 Stat. 328 (1990).

Anderson, L. A., \& Dedrick, R. F. (1990). Development of the Trust in Physician Scale: A measure to assess interpersonal trust in patient-physician relationships. Psychological Reports, 67, 1091-1100. 
Anderson, M. L., Riker, T., Gagne, K., Hakulin, S., Higgins, T., Meehan, J., . . Wolf Craig, K. S. (2018). Deaf Qualitative Health Research: Leveraging Technology to Conduct Linguistically and Sociopolitically Appropriate Methods of Inquiry. Qualitative Health Research, 28(11), 1813-1824. doi:10.1177/1049732318779050

Anderson, M. L., Wolf Craig, K. S., \& Ziedonis, D. M. (2017a). Barriers and facilitators to Deaf trauma survivors' help-seeking behavior: Lessons for behavioral clinical trials research. Journal of Deaf Studies \& Deaf Education, 22(1), 118-130. doi:10.1093/deafed/enw066

Anderson, M. L., Wolf Craig, K. S., \& Ziedonis, D. M. (2017b). Deaf people's helpseeking following trauma: Experiences with and recommendations for the Massachusetts behavioral health care system. Psychological Trauma: Theory, Research, Practice \& Policy, 9(2), 239-248. doi:10.1037/tra0000219

Arksey, H., \& O'Malley, L. (2005). Scoping studies: Towards a methodological framework. International Journal of Social Research Methodology, 8(1), 19-32. doi:10.1080/1364557032000119616

Austin, S. \& McGrath, M. (2006). Telemental Health technology in deaf and general mental-health services: Access and use. American Annals of the Deaf, 151(3), 311-317.

Baier, A. (1986). Trust and antitrust. Ethics, 96(2), 231-260.

Baker-Shenk, C. (1992). The interpreter: Machine, advocate, or ally? In J. Plant-Moeller (Ed.), Expanding Horizons (pp. 120-140). Silver Spring, MD: RID Publications. 
Balch, G. I., \& Mertens, D. M. (1999). Focus Group Design and Group Dynamics: Lessons from Deaf and Hard of Hearing Participants. American Journal of Evaluation, 20(2), 265-277. doi:doi:10.1177/109821409902000208

Barnett, S., \& Franks, P. (1999). Telephone ownership and Deaf people: Implications for telephone surveys. American Journal of Public Health, 89(11), 1754-1756.

Bat-Chava, Y., Martin, D., \& Kosciw, J. G. (2005). Barriers to HIV/AIDS knowledge and prevention among deaf and hard of hearing people. AIDS Care, 17(5), 623634.

Bauman, H. D. L. (2004). Audism: Exploring the Metaphysics of Oppression. Journal of Deaf Studies and Deaf Education, 9(2), 239-246.

Blackstock, O. J., Addison, D. N., Brennan, J. S., \& Alao, O. A. (2012). Trust in primary care providers and antiretroviral adherence in an urban HIV clinic. Journal of Health Care for the Poor and Underseved, 23(1), 88-89. doi:10.1353/hpu.2012.0006.

Blendon, R. J., Benson, J. M., \& Hero, J. O. (2014). Public trust in physicians: U.S. medicine in international perspective. The New England Journal of Medicine, 371(17), 1570-1572. doi:10.1056/NEJMp1407373

Bok, S. (1978). Lying: Moral choice in public and private life. New York City, NY: Pantheon Books.

Boulware, L. E., Cooper, L. A., Ratner, L. E., LaVeist, T. A., \& Powe, N. R. (2003). Race and trust in the health care system. Public Health Reports (1974-), 118(4), $358-365$.

Brannen, N. S. (1993). Translating Style. Japan Quarterly, 40(2), 142-155. 
Buhr, T., \& Blendon, R. J. (2011). Trust in government and healthcare institutions. In R. J. Blendon, M. Brodie, J. M. Benson, \& D. E. Altman (Eds.), American public opinion and health care (pp. 15-38). Washington, D.C.: CQ Press.

Cabral, L., Muhr, K., \& Savageau, J. (2014). Hearing those who can't: Perspectives of the Deaf and hard of hearing on mental health services. Clinician's Research Digest, 32(3), 3-3. doi:10.007/s10597-012-9569-z

Cabral, L., Muhr, K., \& Savageau, J. (2013). Perspectives of people who are deaf and hard of hearing on mental health, recovery, and peer support. Community Mental Health Journal, 49(6), 649-657. doi:10.1007/s10597-012-9569-z

Cappell, M. S. (2009). Universal lessons learned by a gastroenterologist from a deaf and mute patient: The importance of nonverbal communication and establishing patient rapport and trust. American Annals of the Deaf, 154(3), 274-276.

Caterinicchio, R. P. (1979). Testing plausible path models of interpersonal trust in patient-physician treatment relationships. Social Science \& Medicine. Part A: Medical Psychology \& Medical Sociology, 13, 81-99. doi:https://doi.org/10.1016/0271-7123(79)90011-7

Collyer, F. M., Willis, K. F., Lewis, S. D. (2017). Gatekeepers in the healthcare sector: Knowledge and Bourdieu's concept of field. Social Science \& Medicine, 186, 96103. doi:10.1016/j.socscimed.2017.06.004

Crowe, T. (2017). Is telemental health services a viable alternative to traditional psychotherapy for Deaf individuals? Community Mental Health Journal, 53(2), 154-162. doi:10.1007/s10597-016-0025-3 
Daniel J. Edelman Holdings Inc. (2019). Trust in healthcare. Retrieved from https://www.edelman.com/post/trust-in-healthcare-2018

de Paiva, H. K., de Camargo, É. M., de Paula da Silva, A. A., \& Reis, R. S. (2016). Association between intention for physical activity practice, social support and physical activity. Brazilian Journal of Kineanthropometry \& Human Performance, 18(6), 67-677. doi:10.5007/1980-0037.2016v18n6p667

de Waard, J., \& Nida, E. A. (1998). From one language to another: Functional equivalence in Bible translating. New York: United Bible Societies.

Registry of Interpreters for the Deaf. (2005). NAD-RID Code of Professional Conduct. Alexandria, VA: Registry of Interpreters for the Deaf.

Deutsch, M. (1958). Trust and suspicion. Journal of Conflict Resolution, 2(4), 265. Deutsch, M. (1960). The effect of motivational orientation upon trust and suspicion. Human Relations, 13(2), 123-139. doi:10.1177/001872676001300202

DeVinney, J., \& Murphy, S. (2002). Mental health experiences and deafness: Personal and legal perspectives. Psychiatric Rehabilitation Journal, 25(3), 304-309.

EBSCO Information Services. (2020). CINAHL Complete. https://www.ebsco.com/products/research-databases/cinahl-complete Epic Enterprises LLC. (2019). TapeACall pro: Call recorder (Version 4.9.0). TelTech. https://apps.apple.com/us/app/tapeacall-pro-call-recorder/id577499909

Ervas, F. (2014). Chapter 5: On Semantic and Pragmatic Equivalence in Translation. In Translating the DCFR and Drafting the CESL : A Pragmatic Perspective: De Gruyter: Berlin, Boston. 
Feldman, D. M., \& Gum, A. (2007). Multigenerational perceptions of mental health services among deaf adults in Florida. American Annals of the Deaf, 152(4), 391397

Feldman, D., Kluwin, T. N., \& McCrone, W. P. (2005). Deaf clients' perceptions of counseling expertise as a function of counselors' signing skills, gender, and therapy type. American Annals of the Deaf, 150(5), 408-414.

Fellinger, J., Holzinger, D., \& Pollard, R. (2012). Mental health of Deaf people. Lancet, 379(9820), 1037-1044.

Ferguson-Coleman, E., Keady, J., \& Young, A. (2014). Dementia and the Deaf community: Knowledge and service access. Aging \& Mental Health, 18(6), 674682. doi:10.1080/13607863.2014.880405

Frank, A. (2017). Deaf families' unique experiences and obstacles. Journal of Social Work in Disability \& Rehabilitation, 16(3/4), 216-229. doi:10.1080/1536710X.2017.1372238

Gratz, E. F. (2018). An examination of the mediating effect of institutional trust on interpersonal trust and readiness for change in university faculty (10742936). [Doctoral dissertation]. ProQuest Dissertations Publishing, University of La Verne.

Guthmann, D., \& Blozis, S. A. (2001). Unique issues faced by deaf individuals entering substance abuse treatment and following discharge. American Annals of the Deaf, 146(3), 294-304. 
Haines, D. (2020, April 02). Register in English writing and how to control it. Just Publishing Advice. https://justpublishingadvice.com/control-register-in-yourwriting/

Hall, M. A., Dugan, E., Zheng, B., \& Mishra, A. K. (2001). Trust in physicians and medical institutions: What is it, can it be measured, and does it matter? Milbank Quarterly, 79(4), 613-639.

Hall, M. A., Zheng, B., Dugan, E., Camacho, F., Kidd, K. E., Mishra, A., \& Balkrishnan, R. (2002). Measuring patients' trust in their primary care providers. Medical Care Research and Review, 59(3), 293-318. doi:10.1177/1077558702059003004

Hansen, H., Bourgois, P., \& Drucker, E. (2014). Pathologizing poverty: New forms of diagnosis, disability, and structural stigma under welfare reform. Social Science \& Medicine, 103(1), 76-83. doi:10.1016/j.socscimed.2013.06.033

Harmer, L. M. (1999). Health care delivery and Deaf people: Practice, problems, and recommendations for change. Journal of Deaf Studies \& Deaf Education, 4(2), 73-110.

Harris, R., Holmes, H. M., \& Mertens, D. M. (2009). Research Ethics in Sign Language Communities. Sign Language Studies, 9(2), 104-131.

Higashino, Y. (2001). Cultural equivalence: Its effectiveness and complications - Has "white glove" achieved the equivalent effect of "shiro tabi"? Japan Association for Interpreting and Translation Studies, 1(December), 53-63.

Horne, N., \& Pennington, J. (2010). The role of the nurse specialist in the highly specialized field of mental health and deafness. Journal of Psychiatric \& Mental Health Nursing, 17(4), 355-358. doi:10.1111/j.1365-2850.2010.01552.x 
Iezzoni, L. I., Davis, R. B., Soukup, J., \& O'Day, B. (2002). Satisfaction with quality and access to health care among people with disabling conditions. International Journal for Quality in Health Care, 14(5), 369-381. doi:intqhc/14.5.369

Iezzoni, L. I., O'Day, B. L., Killeen, M., \& Harker, H. (2004). Communicating about health care: Observations from persons who are deaf or hard of hearing. Annals of Internal Medicine, 140(5), 356-362.

Jackson, M. (2011). Deafness and antenatal care: Understanding issues with access. British Journal of Midwifery, 19(5), 280-284.

Jacobs, E. A., Mendenhall, E., McAlearney, A. S., Rolle, I., Whitaker, E. E., Warnecke, R., \& Ferrans, C. E. (2011). An exploratory study of how trust in health care institutions varies across African American, Hispanic and White populations. Communication and Medicine, 8(1), 89-98. doi:10.1558/cam.v8i1.89

Jampel, J. B. (2010). When hearing clients work with a deaf therapist. Psychotherapy, 47(2), 144-150. doi:10.1037/a0019753

Jezewski, M. A. (1993). Culture brokering as a model for advocacy. Nurse Health Care, $14(2), 78-85$.

Jin, D., \& Nida, E. A. (2006). On translation: An expanded edition. Hong Kong: City University of Hong Kong Press.

Jones, J., \& Saad, L. (2019). Honesty and ethics survey: December wave one, final topline. Retrieved from https://news.gallup.com/file/poll/274751/200106HonestyEthics.pdf

Kao, A. C., Green, D. C., Davis, N. A., Koplan, J. P., \& Cleary, P. D. (1998). Patients' trust in their physicians: Effects of choice, continuity, and payment method. 
Journal of General Internal Medicine, 13(10), 681-686. doi:10.1046/j.15251497.1998.00204.x

Kaplan, H. (1996). The nature of Deaf culture: Implications for speech and hearing professionals. Journal of the Academy of Rehabilitative Audiology, 29, 71-84.

King, J. F. (2005). Practical considerations for accommodating the deaf patient. Patient Care, 39(1), 17-23.

Kobayashi, Y., Boudreault, P., Hill, K., Sinsheimer, J. S., \& Palmer, C. G. S. (2013). Using a social marketing framework to evaluate recruitment of a prospective study of genetic counseling and testing for the deaf community. BMC Medical Research Methodology, 13(1), 145. doi:10.1186/1471-2288-13-145

Kritzinger, J., Schneider, M., Swartz, L., \& Braathen, S. H. (2014). 'I just answer 'yes' to everything they say': Access to health care for deaf people in Worcester, South Africa and the politics of exclusion. Patient Education \& Counseling, 94(3), 379383. doi: $10.1016 /$ j.pec.2013.12.006

Krueger, R. A., \& Casey, M. A. (2009). Focus groups: A practical guide for applied research (4th ed.). Thousand Oaks, CA: SAGE Publications, Inc.

Lane, H. (1999). The mask of benevolence: Disabling the Deaf community. New York: Knopf.

Lapiak, J. (2020a, April 22). ASL sign for: BENEVOLENCE. Handspeak. https://www.handspeak.com/word/search/index.php?id=8366

Lapiak, J. (2020b, April 22). ASL sign for: INTEGRITY. Handspeak. https://www.handspeak.com/word/search/index.php?id=7180 
Lapinski, J., Colonna, C., Sexton, P., \& Richard, M. (2015). American Sign Language and Deaf culture competency of osteopathic medical students. American Annals of the Deaf, 160(1), 36-47. doi:10.1353/aad.2015.0014

Leisen, B., \& Hyman, M. R. (2001). An improved scale for assessing patients' trust in their physician. Health Marketing Quarterly, 19(1), 23-42. doi:10.1300/J026v19n01_03

Lieu, C. C., Sadler, G. R., Fullerton, J. T., \& Stohlmann, P. D. (2007). Communication strategies for nurses interacting with deaf patients. Medsurg Nursing, 16(4), 239245.

Lincoln, Y. S., \& Guba, E. G. (1985). Naturalistic inquiry. Newbury Park, CA: SAGE Publications.

Long, G., Stinson, M., Kelly, R. R., \& Liu, Y. (1999). The relationship between teacher sign skills and student evaluations of teacher capability. American Annals of the Deaf, 144(5), 354-364.

Lount, R. B., \& Pettit, N. C. (2012). The social context of trust: The role of status. Organizational Behavior and Human Decision Processes, 117(1), 15-23. doi:10.1016/j.obhdp.2011.07.005

Lucy, J. A. (2015). Sapir-Whorf Hypothesis. In J. D. Wright (Ed.), International Encyclopedia of the Social \& Behavioral Sciences (Second Edition) (pp. 903906). Oxford: Elsevier.

Luhmann, N. (1979). Trust and power: Two works. New York City, NY: John Wiley \& Sons. 
Lynch, T. J., Wolfson, D. B., \& Baron, R. J. (2019). A trust initiative in health care: Why and why now? Academic Medicine: Journal of the Association of American Medical Colleges, 94(4), 463-465. doi:10.1097/ACM.0000000000002599

Machado, W. C. A., Machado, D. A., de Figueiredo, N. M. A., Tonini, T., de Miranda, R. S., \& de Oliveira, G. M. B. (2013). Sign Language: How the nursing staff interacts to take care of deaf patients? Revista de Pesquisa: Cuidado e Fundamental, 5(3) 283-292. doi:10.9789/2175-5361.2013v5n3p283

Maddalena, V., O'Shea, F., \& Murphy, M. (2012). Palliative and end-of-life care in Newfoundland's Deaf community. Journal of Palliative Care, 28(2), 105-112.

Manning, K. (1997). Authenticity in constructivist inquiry: Methodological considerations without prescription. Qualitative Inquiry, 3(1), 93-115. doi:10.1177/107780049700300105

Mayer, R. C., Davis, J. H., \& Schoorman, F. D. (1995). An integrative model of organizational trust. Academy of Management Review, 20(3), 709-734. doi:10.5465/AMR.1995.9508080335

McKee, M., Schlehofer, D., \& Thew, D. (2013). Ethical issues in conducting research with deaf populations. American Journal of Public Health, 103(12), 2174-2178. doi:10.2105/AJPH.2013.301343

Meador, H. E., \& Zazove, P. (2005). Health care interactions with Deaf culture. The Journal of the American Board of Family Practice, 18(3), 218-222. doi:10.3122/jabfm.18.3.218

Mechanic, D. (1996). Changing medical organization and the erosion of trust. The Milbank Quarterly, 74(2), 171-189. 
Mechanic, D., \& Meyer, S. (2000). Concepts of trust among patients with serious illness. Social Science \& Medicine, 51(5), 657-668. doi:https://doi.org/10.1016/S02779536(00)00014-9

Microsoft. (2019). Microsoft Word for Mac (Version 16). https://www.microsoft.com/enus/microsoft-365/word

Middleton, A., Hewison, J., \& Mueller, R. F. (1998). Attitudes of deaf adults toward genetic testing for hereditary deafness. American Journal of Human Genetics, 63(4), 1175-1180. doi:10.1086/302060

Middleton, A., Emery, S. D., \& Turner, G. H. (2010). Views, knowledge, and beliefs about genetics and genetic counseling among deaf people. Sign Language Studies, 10(2), 170-196.

Middleton, A., Turner, G. H., Bitner-Glindzicz, M., Lewis, P., Richards, M., Clarke, A., \& Stephens, D. (2010). Preferences for communication in clinic from Deaf people: A cross-sectional study. Journal of Evaluation in Clinical Practice, 16(4), 811-817. doi:10.1111/j.1365-2753.2009.01207.x

Miller, M. S. (2010). Epistemology and people who are deaf: deaf worldviews, views of the deaf world, or my parents are hearing. American Annals of the Deaf, 154(5), 479-485.

Mogensen, L., \& Mason, J. (2015). The meaning of a label for teenagers negotiating identity: Experiences with autism spectrum disorder. Sociology of Health \& Illness, 37(2), 255-269. doi:10.1111/1467-9566.12208

Moore, M. S., \& Levitan, L. (2011). For hearing people only (3rd ed.). Rochester, NY: Deaf Life Press. 
Mprah, W. K. (2013). Perceptions about barriers to sexual and reproductive health information and services among Deaf people in Ghana. Disability, $C B R \&$ Inclusive Development, 24(3), 21-36. doi:10.5463/DCID.v24i3.234

Mprah, W. K., Anafi, P., \& Yeaboah, P. Y. A. (2017). Exploring misinformation of family planning practices and methods among deaf people in Ghana. Reproductive Health Matters, 25(50), 20-30.

doi:10.1080/09688080.2017.1332450

Müller, E., Zill, J. M., Dirmaier, J., Härter, M., \& Scholl, I. (2014). Assessment of trust in physician: A systematic review of measures. PLoS ONE, 9(9), e106844. doi:10.1371/journal.pone.0106844

Munro, L., Knox, M., \& Lowe, R. (2008). Exploring the potential of constructionist therapy: Deaf clients, hearing therapists and a reflecting team. Journal of Deaf Studies \& Deaf Education, 13(3), 307-323.

Murray, B., \& McCrone, S. (2015). An integrative review of promoting trust in the patient-primary care provider relationship. Journal of Advanced Nursing, 71(1), 3-23. doi:10.1111/jan.12502

Myers, L. L., \& Thyer, B. A. (1997). Social work practice with deaf clients: Issues in culturally competent assessment. Social Work in Health Care, 26(1), 61-76.

Myers, S. S., \& Fernandes, J. K. (2010). Deaf Studies: A Critique of the Predominant U.S. Theoretical Direction. Journal of Deaf Studies and Deaf Education, 15(1), $30-49$.

Nagakura, H., Schneider, G., Morris, J., Lafferty, K. A., \& Palmer, C. G. S. (2015). Assessing deaf awareness training: Knowledge and attitudes of recent genetic 
counseling graduates. Journal of Genetic Counseling, 24(1), 104-116.

doi:10.1007/s10897-014-9742-3

Napier, J., \& Kidd, M. R. (2013). English literacy as a barrier to health care information for deaf people who use Auslan. Australian Family Physician, 42(12), 896-899.

Nida, E. A. (1964). Toward a science of translating: With special reference to principles and procedures involved in Bible translating. Leiden: E. J. Brill.

Nonaka, A. M. (2016). Legal and ethical imperatives for using certified Sign Language interpreters in health care settings: How to 'do no harm' when 'its (all) Greek' (Sign Language) to you. Care Management Journals, 17(3), 114-128. doi:10.1891/1521-0987.17.3.114

O'Malley, A. S., Forrest, C. B., \& Mandelblatt, J. (2002). Adherence of low-income women to cancer screening recommendations: The roles of primary care, health insurance, and HMOs. Journal of General Internal Medicine, 17(2), 144-154. doi:10.1046/j.1525-1497.2002.10431.x

Orrie, S., \& Motsohi, T. (2018). Challenges experienced by healthcare workers in managing patients with hearing impairment at a primary health care setting: A descriptive case study. South African Family Practice, 60(6), 207-211. doi:10.1080/20786190.2018.1507566

Ozawa, S., \& Sripad, P. (2013). How do you measure trust in the health system? A systematic review of the literature. Social Science Medicine, 91, 10-14. doi:10.1016/j.socscimed.2013.05.005

Patton, M. Q. (1990). Qualitative evaluation and research methods (2nd ed.). Newbury Park, CA: Sage Publications. 
Pellowski, J. A., Price, D. M., Allen, A. M., Eaton, L. A., \& Kalichman, S. C. (2017). The differences between medical trust and mistrust and their respective influences on medication beliefs and ART adherence among African-Americans living with HIV. Psychology Health, 32(9), 1127-1139.

doi:10.1080/08870446.2017.1324969.

Penman, D. T., Holland, J. C., Bahna, G. F., Morrow, G., Schmale, A. H., Derogatis, L. R., Carnrike, C. L., \& Cherry, R. (1984). Informed consent for investigational chemotherapy: Patients' and physicians' perceptions. Journal of Clinical Oncology, 2(7), 849-855.

Pereira, P. C. A., \& Fortes, P. A. C. (2010). Communication and information barriers to health assistance for deaf patients. American Annals of the Deaf, 155(1), 31-37.

Platt, J. E., Jacobson, P. D., \& Kardia, S. L. R. (2018). Public trust in health information sharing: A measure of system trust. Health Services Research, 53(2), 824-845. doi:10.1111/1475-6773.12654

Plumridge, G., Redwood, S., Greenfield, S., Akhter, N., Chowdhury, R., Khalade, A., \& Gill, P. (2012). Involving interpreters in research studies. Journal of Health Services Research \& Policy, 17(3), 190-192.

Poetry Foundation. (2020, April, 24). To a Mouse by Robert Burns. https://www.poetryfoundation.org/poems/43816/to-a-mouse-56d222ab36e33

Polit, D. F., \& Beck, C. T. (2012). Resource manual for nursing research: Generating and assessing evidence for nursing practice (9th ed. ed.). Philadelphia: Wolters Kluwer Health/Lippincott Williams \& Wilkins. 
Pollard, R. (2002). Ethical conduct in research involving Deaf people. In V. Gutman (Ed.), Ethics in Mental Health and Deafness (pp. 162-178). Washington, D.C.: Gallaudet University Press.

Pollard, R. Q. (1992). Cross-cultural ethics in the conduct of deafness research. Rehabilitation Psychology, 37(2), 87-101.

Reader, D., Foulkes, H., \& Robinson, C. (2017). Investigating barriers to mental health care experienced by the Deaf community in North Wales. Mental Health Nursing, $37(3), 14-19$.

Reagan, T. (1995). A sociocultural understanding of deafness: American Sign Language and the culture of Deaf people. International Journal of Intercultural Relations, 19(2), 239-251. doi:10.1016/0147-1767(95)00007-X

Reeves, D., \& Kokoruwe, B. (2005). Communication and communication support in primary care: A survey of deaf patients. Audiological Medicine, 3(2), 95-107.

Robins, R., \& Mangan, M. (1999). Seen and not heard. Nursing Times, 95(37), 30-32.

Rørtveit, K., Hansen, B. S., Leiknes, I., Joa, I., Testad, I., \& Severinsson, E. (2015). Patient's expereinces of trust in the patient-nurse relationship: A systematic review of qualitative studies. Open Journal of Nursing, 5, 195-209. doi:10.4236/ojn.2015.53024

Rose, A., Peters, N., Shea, J. A., \& Armstrong, K. (2004). Development and testing of the health care system distrust scale. Journal of General Internal Medicine, 19(1), 5763. doi:10.1111/j.1525-1497.2004.21146.x 
Rosen, R. G. (2000). Perspectives of the deaf community on early identification and intervention: A case for diversity and partnerships. Seminars in Hearing, 21(4), $327-428$.

Russell, S. (2005). Treatment-seeking behaviour in urban Sri Lanka: Trusting the state, trusting private providers. Social Science \& Medicine, 61(7), 1396-1407. doi:https://doi.org/10.1016/j.socscimed.2004.11.077

Schoenthaler, A., Montague, E., Manwell, L. B., Brown, R., Schwartz, M. D., \& Linzer, M. (2014). Patient-physician racial/ethnic concordance and blood pressure control: The role of trust and medication adherence. Ethnicity \& Health, 19(5), 565-578. doi:10.1080/13557858.2013.857764

Schoorman, F. D., Mayer, R. C., \& Davis, J. H. (2007). An integrative model of organizational trust: Past, present, and future. The Academy of Management Review, 32(2), 344-354.

Shaw, R. (1987). Determining Register in Sign-to-English Interpreting. Sign Language Studies, 1057(1), 295-322. doi:10.1353/sls.1987.0019

Sheppard, K. (2011a). Using American Sign Language interpreters to facilitate research among deaf adults: Lessons learned. Journal of Transcultural Nursing, 22(2), 129-134. doi:10.1177/1043659610395765

Sheppard, K. (2011b). Using American Sign Language interpreters to facilitate research among Deaf adults: Lessons learned. Journal of Transcultural Nursing, 22(2), 129-134. doi:10.1177/1043659610395765 
Singleton, J. L., Jones, G., \& Hanumantha, S. (2014). Toward Ethical Research Practice With Deaf Participants. Journal of Empirical Research on Human Research Ethics: An International Journal, 9(3), 59-66.

Straten, G. F., Friele, R. D., \& Groenewegen, P. P. (2002). Public trust in Dutch health care. Social Science \& Medicine, 55(2), 227-234.

Stebnicki, J. A., \& Coeling, H. V. (1999). The culture of the Deaf. Journal of Transcultural Nursing, 10(4), 350-357. doi:10.1177/104365969901000413

Steinberg, A. G., Barnett, S., Meador, H. E., Wiggins, E. A., \& Zazove, P. (2006). Health care system accessibility: Experiences and perceptions of Deaf people. Journal of General Internal Medicine, 21(3), 260-266. doi:10.1111/j.15251497.2006.00340.x

Steinberg, A. G., Sullivan, V. J., \& Loew, R. C. (1998). Cultural and linguistic barriers to mental health service access: The Deaf consumer's perspective. American Journal of Psychiatry, 155(7), 982-984. doi:10.1176/ajp.155.7.982

Steinberg, A. G., Wiggins, E. A., Barmada, C. H., \& Sullivan, V. J. (2002). Deaf women: Experiences and perceptions of healthcare system access. Journal of Women's Health (15409996), 11(8), 729-741. doi:10.1089/15409990260363689

Stepanikova, I., Mollborn, S., Cook, K., Thom, D., \& Kramer, R. (2006). Patients' race, ethnicity, language, and trust in a physician. Journal of Health and Social Behavior, 47(4), 390-405.

Thom, D. H., Hall, M. A., \& Pawlson, L. G. (2004). Measuring patients' trust in physicians when assessing quality of care. Health Affairs, 23(4), 124-132. doi:10.1377/hlthaff.23.4.124 
Thom, D. H., Ribisl, K. M., Stewart, A. L., \& Luke, D. A. (1999). Further validation and reliability testing of the trust in physician scale. Medical Care, 37(5), 510-517.

Thu, J., \& Huang, J. (2014). Bridging the gap: Effective communication despite barriers. California Pharmacist, 61(3), 21-25.

Tran, D. Q., Barry, V., Antun, A., Ribeiro, M., Stein, S., \& Kempton, C. L. (2017). Physician trust and depression influence adherence to factor replacement: A single-centre cross-sectional study. Haemophilia, 23(1), 98-104. doi:10.1111/hae.13078

Tricco, A. C., Lillie, E., Zarin, W., O’Brien, K. K., Colquhoun, H., Levac, D. ... Moher, D. (2018). PRISMA extension for scoping reviews (PRISMAScR): Checklist and explanation. Annals of Internal Medicine, 169(7), 467-473. doi:10.7326/M180850

Trudgill, P. (1983). On dialect: Social and geographical perspectives. New York: New York University Press.

Ubido, J., Huntington, J., \& Warburton, D. (2002). Inequities in access to healthcare faced by women who are deaf. Health and Social Care in the Community, 10(4), 247-253. doi:10.1046/j.1365-2524.2002.00365.x

Valente, J. M., \& Danforth, S. (2016). Life in inclusive classrooms: Storytelling with disability studies in education (Vol. 36). Bank Street Occasional Paper Series: Bank Street College of Education.

van Velsen, L., Tabak, M., \& Hermens, H. (2017). Measuring patient trust in telemedicine services: Development of a survey instrument and its validation for 
an anticoagulation web-service. International Journal of Medical Informatics, 97, $52-58$.

VanPuymbrouck, L., Friedman, C., \& Feldner, H. (2020). Explicit and implicit disability attitudes of healthcare providers. Rehabilitation Psychology, 65(2), 101-112. doi:10.1037/rep0000317

Vermeulen, A. M., van Bon, W., Schreuder, R., Knoors, H., \& Snik, A. (2007). Reading comprehension of deaf children with cochlear implants. Journal of Deaf Studies \& Deaf Education, 12(3), 283-302.

Wang, H., Schlesinger, M., Wang, H., \& Hsiao, W. C. (2009). The flip-side of social capital: The distinctive influences of trust and mistrust on health in rural China. Social Science \& Medicine, 68(1), 133-142. doi:10.1016/j.socscimed.2008.09.038

White, R. O., Osborn, C., .Y., Gebretsadik, T., Kripalani, S., \& Rothman, R. L. (2013). Health literacy, physician trust, and diabetes-related self-care activities in Hispanics with limited resources. Journal of Health Care for the Poor and Underseved, 24(4), 1756-1768. doi:10.1353/hpu.2013.0177

Witko, J., Boyles, P., Smiler, K., \& McKee, R. (2017). Deaf New Zealand Sign Language users' access to healthcare. New Zealand Medical Journal, 130(1466), 53-61.

Wolsey, J. A., Dunn, K. M., Gentzke, S. W., Joharchi, H. A., \& Clark, M. D. (2017). Deaf/Hearing Research Partnerships. American annals of the deaf, 161(5), 571582. doi:10.1353/aad.2017.0007

Woodroffe, T., Gorenflo, D. W., Meador, H. E., \& Zazove, P. (1998). Knowledge and attitudes about AIDS among deaf and hard of hearing persons. AIDS Care, 10(3), 377-386. 
Wright, D. (1993). Deaf people's perceptions of communication with nurses. British Journal of Nursing, 2(11), 567-571.

Young, A. M., Ackerman, J., \& Kyle, J. G. (2000). On creating a workable signing environment: Deaf and hearing perspectives. Journal of Deaf Studies \& Deaf Education, 5(2), 186-195.

Young, A., Ferguson-Coleman, E., \& Keady, J. (2018). Authentic public and patient involvement with Deaf sign language users: It is not just about language access. Dementia, 17(8), 1001-1010. doi:10.1177/1471301218789567

Young, A., Ferguson-Coleman, E., \& Keady, J. (2016). Understanding dementia: Effective information access from the Deaf community's perspective. Health $\&$ Social Care in the Community, 24(1), 39-47. doi:10.1111/hsc.12181

Zhao, D-H., Rao, K-Q., \& Zhang, Z-R. (2016). Patient trust in physicians: Empirical evidence from Shanghai, China. Chinese Medical Journal, 129(7), 814-818. doi:10.4103/0366-6999.178971 


\section{Appendix A}

Figure 1

Proposed Model of Trust

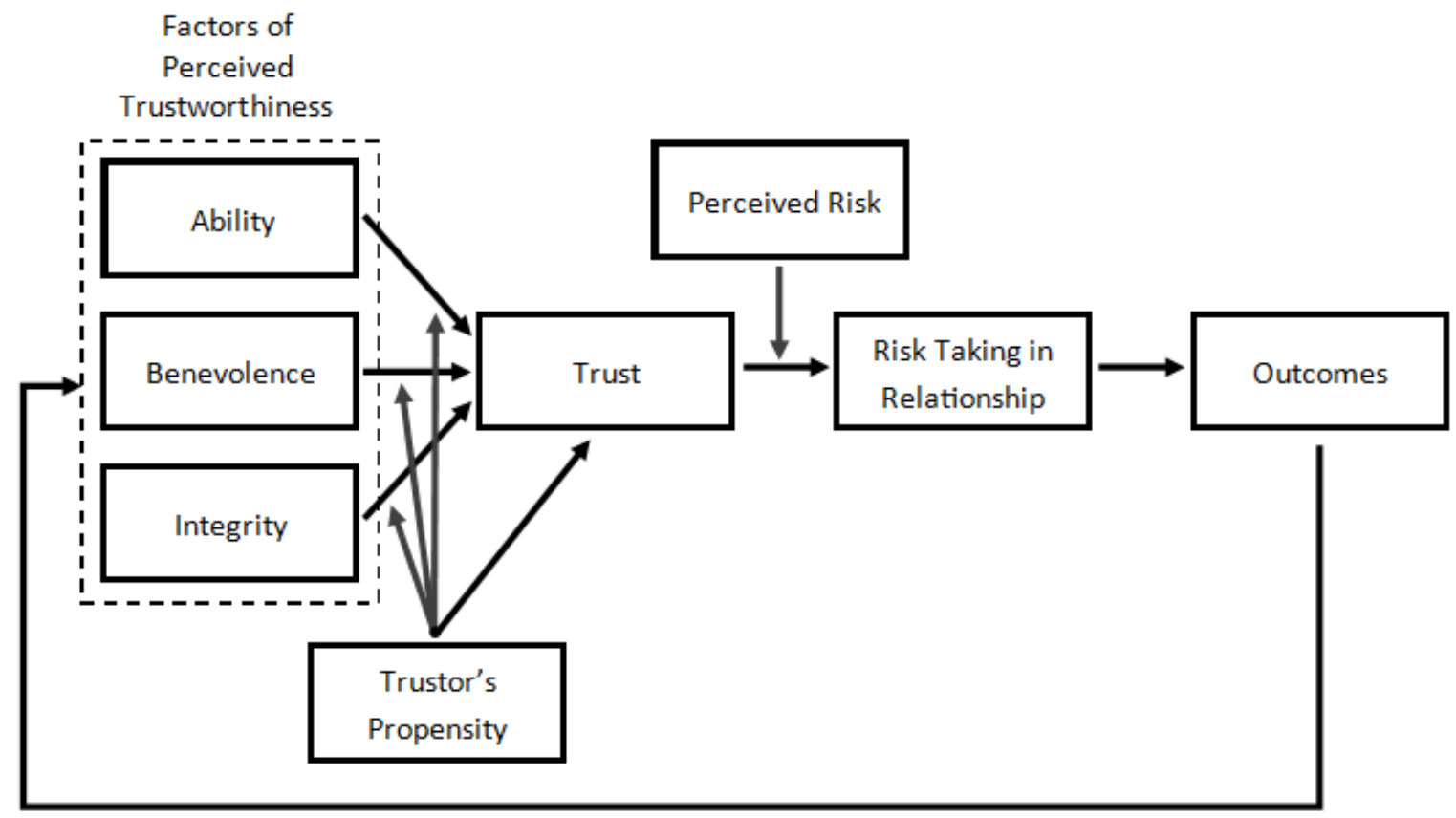

Note. Reproduced from Mayer et al. (1995) 


\section{Appendix B}

Figure 2

Trust Components of the Proposed Model of Trust

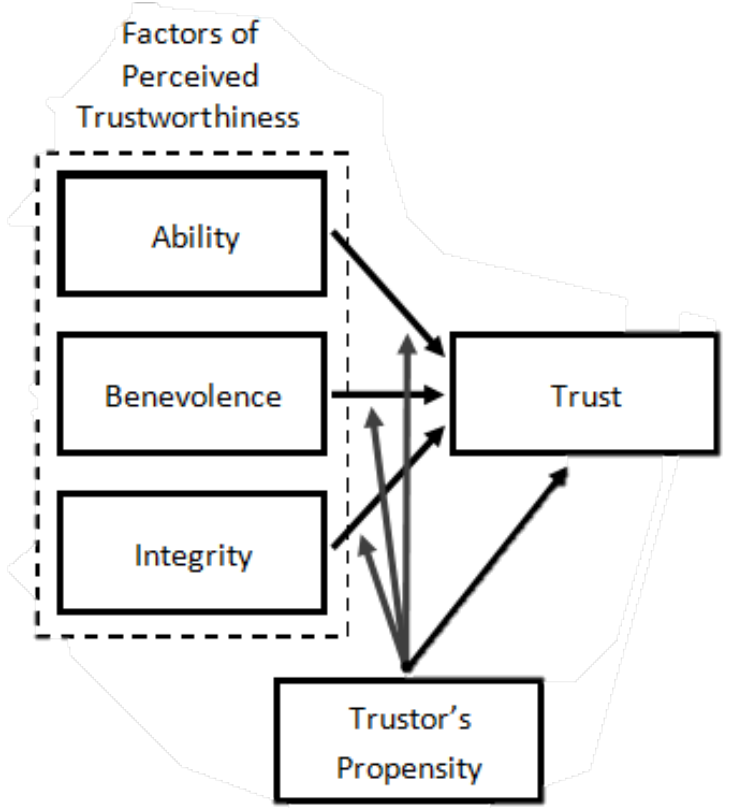

Note. Reproduced from Mayer et al. (1995) 


\section{Appendix C}

\section{Table 1}

Citation Identification, Screening, Review, and Inclusion by Order of Search Terms Used

Search Terms

\begin{tabular}{|c|c|c|c|c|c|c|c|c|c|c|c|c|c|c|c|}
\hline Phases & $\underset{E}{E}$ & $\stackrel{*}{\stackrel{*}{\rightleftarrows}}$ & 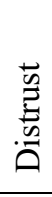 & $\stackrel{\tilde{n}}{\stackrel{\tilde{n}}{E}}$ & 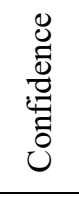 & 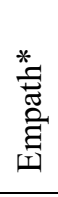 & 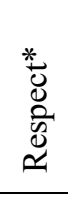 & $\begin{array}{l}* \\
.0 \\
\stackrel{0}{0} \\
\tilde{\omega}\end{array}$ & 兽 & 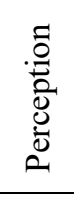 & 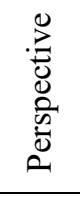 & $\stackrel{*}{\stackrel{*}{\partial}}$ & 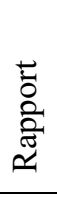 & 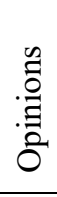 & 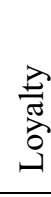 \\
\hline Identified & 12 & 20 & 1 & 4 & 120 & 14 & 146 & 6 & 542 & 746 & 234 & 35 & 2 & 29 & 1 \\
\hline Screened & 11 & 8 & 1 & 4 & 119 & 12 & 139 & 6 & 498 & 591 & 128 & 20 & 1 & 14 & 1 \\
\hline Eligible & 6 & 7 & - & 4 & 7 & 5 & 6 & 1 & 50 & 21 & 20 & 5 & 1 & 1 & - \\
\hline Reviewed & 6 & 7 & - & 4 & 7 & 4 & 5 & 1 & 46 & 16 & 19 & 5 & 1 & 1 & - \\
\hline Excluded & 1 & 1 & - & - & 5 & 2 & 1 & 1 & 24 & 6 & 10 & 2 & - & - & - \\
\hline
\end{tabular}

Note. $(*)$ indicates truncated search term. Citations identified in the reference lists of reviewed articles not included. See Appendix E for number of citations and articles in each phase. Duplicates included to show multiple retrieval options. 


\section{Appendix D}

Table 2

Data Charting Form (DCF) Columns by Processing Order of Data

\section{DCF Pre-Sets}

\begin{tabular}{|c|c|}
\hline $\begin{array}{l}\text { EndNote } \\
\text { Fields }\end{array}$ & $\begin{array}{c}\text { Processing } \\
\text { Stage }\end{array}$ \\
\hline ID \# & Title \\
\hline Author & Abstract \\
\hline Year & Article \\
\hline Title & \\
\hline Abstract & \\
\hline
\end{tabular}

Data Exclusion / Inclusion Categories in Order

\begin{tabular}{|c|c|c|c|}
\hline \multicolumn{2}{|c|}{ Title / Abstract Review } & \multicolumn{2}{|c|}{ Full-text Review } \\
\hline $\begin{array}{c}\text { Not About } \\
\text { Deaf People }\end{array}$ & $\begin{array}{c}\text { Deaf People, } \\
\text { No Trust }\end{array}$ & $\begin{array}{l}\text { Deaf People, } \\
\text { Maybe Trust }\end{array}$ & $\begin{array}{l}\text { Sub. / Cursory } \\
\text { Concepts }^{\mathrm{d}}\end{array}$ \\
\hline Cochlear Implants & Cognition & No Trust ${ }^{\text {ae }}$ & Ability \\
\hline Deaf-Blind Persons & Education & Substantive Trust & Comfort \\
\hline Disabled Persons & Epistemology & Cursory Trust & Communication \\
\hline Experts (Health) & Experiences & Sub. Concepts ${ }^{\mathrm{d}}$ & Condescension \\
\hline Experts (Other) & H. Outcomes & Cursory Concepts ${ }^{\mathrm{d}}$ & Confidence \\
\hline Hearing Persons & Knowledge & & Confidentiality \\
\hline Interpreters (ASL) & Technology & & Cultural Comp. \\
\hline Parents \& Family & Social Inter. & & Rapport \\
\hline & & & Respect \\
\hline & & & Satisfaction \\
\hline & & & Understanding \\
\hline
\end{tabular}

${ }^{\mathrm{a}}$ Citation exclusion criteria.

'If no exclusion criteria identified under 'Not About Deaf People', citation reviewed for additional exclusion criteria. 'If no exclusion criteria found under 'Title / Abstract Review', full-text reviewed for trust and trust-related concepts.

'Trust-related concepts further delineated under 'Sub. / Cursory Concepts'.

'Citations recategorized under most appropriate 'Not About Deaf People' or 'Deaf People, No Trust' category. 


\section{Appendix E}

\section{Figure 3}

\section{Flow Diagram of Study Selection}

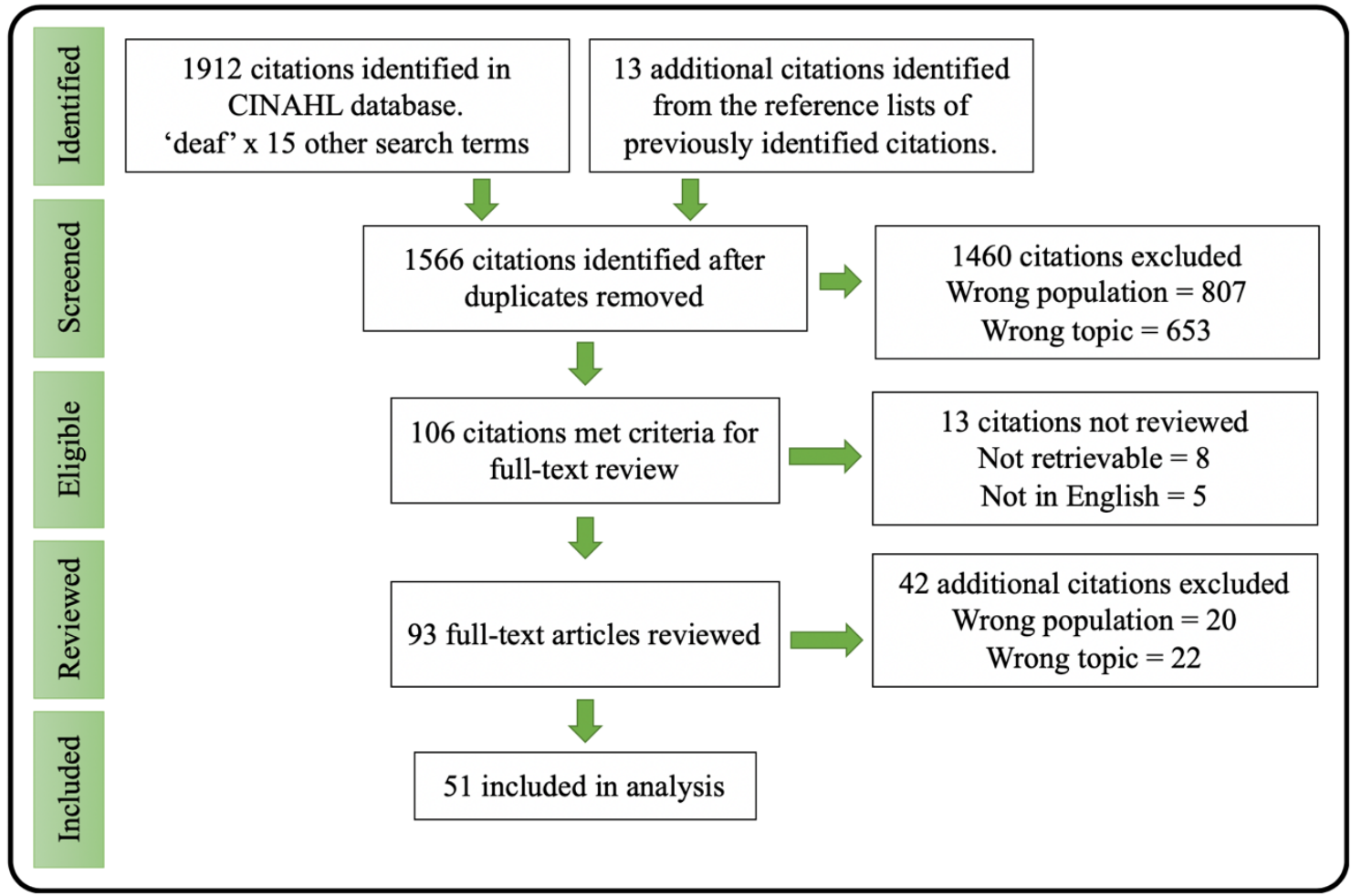




\section{Appendix F}

Table 3

Articles with Substantive and Cursory Content Related to Trust

\section{In-Text Citation \\ Anderson et al., 2017a \\ Anderson et al., 2017b \\ Austen \& McGrath, 2006}

Cacsar 2013

Cabral et al., 2014

Cabral et al., 2013

Cappell, 2009

DeVinney \& Murphy,

2002

Feldman et al., 2005

Feldman \& Gum, 2007

Fellinger et al., 2012

Ferguson-Coleman et

al., 2014

Frank, 2017

Guthmann \& Blozis, 2001

Harmer, 1999

Horne \& Pennington,

2010

Iezzoni et al., 2002

Iezzoni et al., 2004

Jackson, 2011

Jampel, 2010

King, 2005

Kobayashi et al., 2013

Kritzinger et al., 2014

Long et al., 1999

Maddalena et al., 2012

Machado et al., 2013

McKee et al., 2013

Meador \& Zazove,

2005

Middleton et al., 1998

Middleton, Emery, et

al., 2010
Trust

\begin{tabular}{|c|c|}
\hline Substantive & Cursory $^{\mathrm{a}}$ \\
\hline $\mathrm{O}$ & - \\
\hline $\mathrm{O}$ & - \\
\hline- & - \\
\hline
\end{tabular}

Trust-Related

\begin{tabular}{ccc}
\hline Substantive & & Cursory \\
\cline { 1 - 1 } $\mathrm{O}$ & & $\mathrm{X}$ \\
$\mathrm{O}$ & & $\mathrm{X}$ \\
$\mathrm{O}$ & & $\mathrm{X}$
\end{tabular}

$\mathrm{O}$

$\mathrm{O}$

$\mathrm{O}$

$\mathrm{O}$

$\mathrm{O}$

O X

$\mathrm{O}$

-

$\mathrm{O}$

O X

-

$\mathrm{X}$

$\mathrm{X}$

$\mathrm{X}$

O X

$\mathrm{O} \quad \mathrm{X}$

O X

O X

$\mathrm{O} \quad \mathrm{X}$

- $\mathrm{X}$

- X

$\begin{array}{ll}- & X \\ - & X\end{array}$

- X

O X

- X

$\mathrm{O} \quad \mathrm{X}$

- $\quad X$

O X 


\section{In-Text Citation}

Middleton, Turner, et

al., 2010

Mprah, 2013

Mprah et al., 2017

Munro et al., 2008

Myers \& Thyer, 1997

Napier \& Kidd, 2013

Nonaka, 2016

Pereira \& Fortres,

2010

Reader et al., 2017

Reeves \& Kokoruwe, 2005

Robins \& Mangan, 1999

Rosen, 2000

Steinberg et al., 2006

Steinberg et al., 1998

Steinberg et al., 2002

Thu \& Huang, 2014

Witko et al., 2017

Woodroffe et al., 1998

Wright, 1993

Young et al., 2000

Young et al., 2018

Young et al., 2016

Total
Trust Substantive
Trust-Related

Substantive Cursory

$\begin{array}{ll}- & - \\ - & - \\ - & - \\ - & X \\ - & - \\ - & X \\ - & X \\ - & X \\ - & X \\ - & X\end{array}$

$\mathrm{O}$

X

- $\quad X$

- $\mathrm{X}$

- $\quad X$

O X

- $\quad X$

- X

- X

- $\quad X$

O X

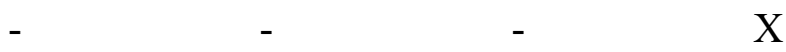

\begin{tabular}{cccc}
- & - & - & $\mathrm{X}$ \\
$\mathrm{X}$ & - & $\mathrm{X}$ \\
$\mathrm{X}$ & - & $\mathrm{X}$ \\
$\mathrm{X}$ & $\mathrm{O}$ & $\mathrm{X}$ \\
- & - & $\mathrm{X}$ \\
- & - & $\mathrm{O}$ & $\mathrm{X}$ \\
$\mathrm{X}$ & - & $\mathrm{X}$ \\
& - & $\mathrm{O}$ & $\mathrm{X}$ \\
- & - & $\mathrm{O}$ & $\mathrm{X}$ \\
& $\mathrm{X}$ & $\mathrm{O}$ & $\mathrm{X}$ \\
\hline 1 & $\mathrm{X}$ & 28 & 51
\end{tabular}

aArticles referencing organizational 'trusts' or 'trustworthiness' of data not included. 


\section{Appendix G}

Table 4

Frequency of Trust-Related Concepts in Patient-Provider Relationships

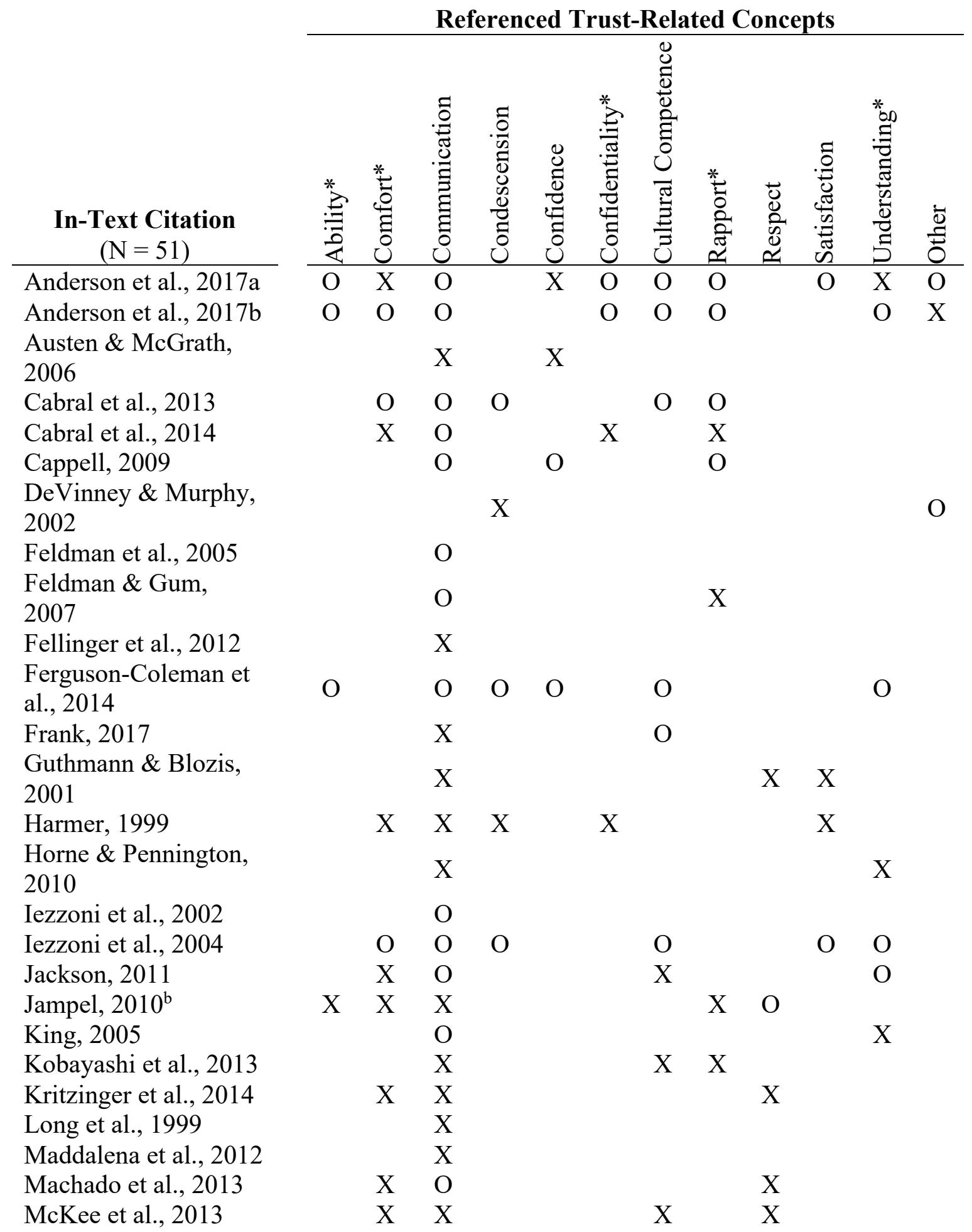




\section{Referenced Trust-Related Concepts}

\begin{tabular}{|c|c|c|c|c|c|c|c|c|c|c|c|c|}
\hline $\begin{array}{l}\text { In-Text Citation } \\
(\mathrm{N}=51)\end{array}$ & 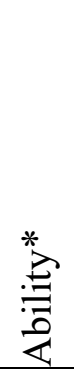 & 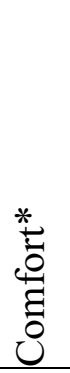 & 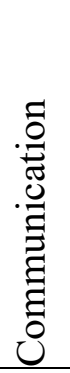 & 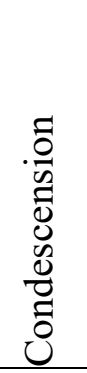 & 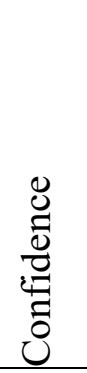 & 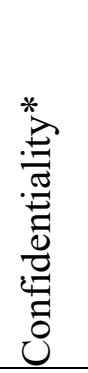 & 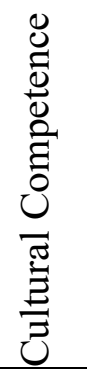 & 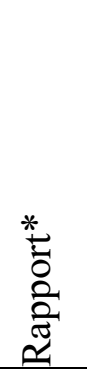 & 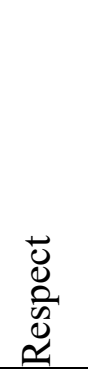 & 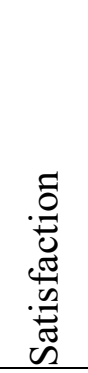 & 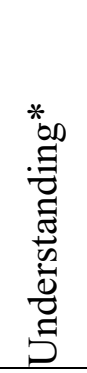 & $\begin{array}{l}\dot{ \pm} \\
\overrightarrow{0}\end{array}$ \\
\hline $\begin{array}{l}\text { Meador \& Zazove, } \\
2005\end{array}$ & & & $\mathrm{O}$ & & & $\mathrm{O}$ & & & & & & \\
\hline Middleton et al., 1998 & & & & & & & $\mathrm{X}$ & & & & & \\
\hline $\begin{array}{l}\text { Middleton, Emery, et } \\
\text { al., } 2010\end{array}$ & & & $\mathrm{O}$ & & & & $\mathrm{X}$ & & & & $\mathrm{X}$ & \\
\hline $\begin{array}{l}\text { Middleton, Turner, et } \\
\text { al., } 2010\end{array}$ & & & & & & $\mathrm{X}$ & $\mathrm{O}$ & & & & & \\
\hline Mprah, 2013 & & $\mathrm{X}$ & & & & $\mathrm{X}$ & $\mathrm{X}$ & & & & & \\
\hline Mprah et al., 2017 & & $X$ & $\mathrm{X}$ & & & & & & $X$ & & $\mathrm{X}$ & \\
\hline Munro et al., 2008 & & $\mathrm{X}$ & $\mathrm{X}$ & & & & $\mathrm{X}$ & $\mathrm{X}$ & & & $\mathrm{X}$ & \\
\hline Myers \& Thyer, 1997 & & & $\mathrm{O}$ & & & & $\mathrm{X}$ & $\mathrm{O}$ & & & & \\
\hline Napier \& Kidd, 2013 & & $\mathrm{X}$ & & & $\mathrm{X}$ & & $\mathrm{X}$ & & & & & \\
\hline Nonaka, 2016 & & & $\mathrm{X}$ & & & & & & & $\mathrm{X}$ & $\mathrm{X}$ & \\
\hline $\begin{array}{l}\text { Pereira \& Fortres, } \\
2010\end{array}$ & & & $\mathrm{X}$ & & & & $\mathrm{X}$ & & $\mathrm{X}$ & & $\mathrm{X}$ & \\
\hline Reader et al., 2017 & $\mathrm{X}$ & $\mathrm{X}$ & & & & & $\mathrm{X}$ & & & & $\mathrm{X}$ & \\
\hline $\begin{array}{l}\text { Reeves \& Kokoruwe, } \\
2005\end{array}$ & $\mathrm{X}$ & & $\mathrm{O}$ & & $\mathrm{O}$ & & & & $\mathrm{X}$ & $\mathrm{X}$ & $\mathrm{O}$ & $\mathrm{X}$ \\
\hline $\begin{array}{l}\text { Robins \& Mangan, } \\
1999\end{array}$ & & $\mathrm{X}$ & $\mathrm{X}$ & & & & & & & & & \\
\hline Rosen, 2000 & & & & & & & & & $\mathrm{X}$ & & & \\
\hline Steinberg et al., 2006 & & $\mathrm{X}$ & $\mathrm{X}$ & & & & & & & & & \\
\hline Steinberg et al., 1998 & & $\mathrm{X}$ & $\mathrm{X}$ & $\mathrm{X}$ & & $\mathrm{X}$ & & & & & & \\
\hline Steinberg et al., 2002 & $\mathrm{O}$ & & $\mathrm{O}$ & & & & & & & & $\mathrm{O}$ & \\
\hline Thu \& Huang, 2014 & & & $\mathrm{X}$ & & & & & & & & & \\
\hline Witko et al., 2017 & & & $\mathrm{O}$ & & & & & & & & $\mathrm{O}$ & $X$ \\
\hline Woodroffe et al., 1998 & & & $\mathrm{X}$ & & & & $\mathrm{X}$ & & & & $\mathrm{O}$ & \\
\hline Wright, 1993 & & & $\mathrm{X}$ & & & & $\mathrm{X}$ & & & & $\mathrm{X}$ & \\
\hline Young et al., 2000 & $\mathrm{X}$ & & $\mathrm{X}$ & & $\mathrm{O}$ & $\mathrm{X}$ & & & $\mathrm{O}$ & & & \\
\hline Young et al., 2018 & & & $\mathrm{X}$ & & & & & & $\mathrm{O}$ & & & \\
\hline Young et al., 2016 & & & & & & & $\mathrm{O}$ & & & & $\mathrm{X}$ & $\mathrm{X}$ \\
\hline Total Count & 8 & 19 & 43 & 6 & 7 & 9 & 21 & 10 & 11 & 6 & 19 & \\
\hline
\end{tabular}

Note. ( *) indicates a composite term. ( ' $\mathrm{O}$ ' ) indicates substantive content. ( ' $\mathrm{X}$ ') indicates cursory content. If both ' $\mathrm{O}$ ' and ' $\mathrm{X}$ ' in same box, only ' $\mathrm{O}$ ' is recorded. ${ }^{a}$ Discussed trust in more detail than other articles

${ }^{\mathrm{b}}$ Article about Deaf psychologist and hearing clients. 


\section{Appendix H}

Figure 4

Focus Group Set-Up

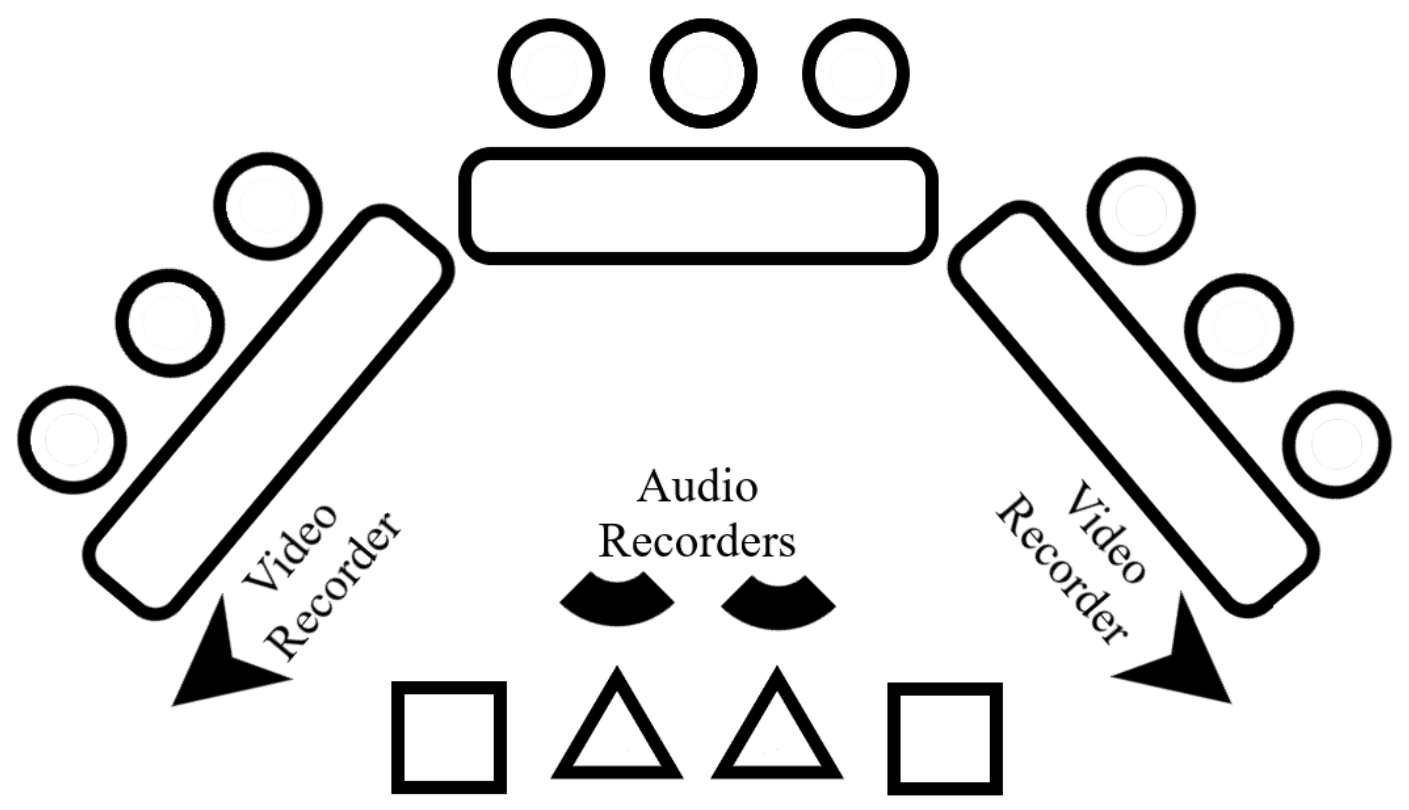

Note. Circles represent participants. Squares represent facilitators. Triangles represent interpreters. 


\section{Appendix I}

\section{Table 5}

Participant Demographics

\begin{tabular}{|c|c|c|c|c|c|}
\hline \multicolumn{6}{|c|}{ Demographics } \\
\hline Gender & $n$ & Race $^{a}$ & $n$ & Age & $n$ \\
\hline Male & $\overline{9}$ & White & $\overline{6}$ & $18-29$ & 2 \\
\hline Female & 1 & Hispanic 30-39 & 3 & $30-39$ & 2 \\
\hline & & Black 40-49 & 1 & $40-49$ & 1 \\
\hline & & Native Amer. & 1 & $50-59$ & 2 \\
\hline & & & & $60+$ & 1 \\
\hline & & & & Undisclosed & 1 \\
\hline
\end{tabular}

\begin{tabular}{|c|c|c|c|c|c|}
\hline \multicolumn{6}{|c|}{ Education and Age of Onset } \\
\hline Education & $n$ & Schooling & $n$ & Age of Deafness & $n$ \\
\hline Less than High School & 1 & Deaf Schools & 5 & Birth & 7 \\
\hline High School or GRE & 5 & Mainstream & 3 & Pre-lingual $(<1)$ & 1 \\
\hline Associate & 3 & Both & 2 & Post-lingual $(>1)$ & 1 \\
\hline Bachelor & 0 & & & Undisclosed & 1 \\
\hline Master & 1 & & & & \\
\hline
\end{tabular}

$\begin{array}{ccc}\text { Deaf } & \begin{array}{c}\text { Cultural Identity } \\ \text { Bicultural }\end{array} & \frac{\text { Hearing }}{n=2}\end{array}$

\begin{tabular}{|c|c|c|c|c|c|}
\hline \multicolumn{6}{|c|}{ Primary Care Provider Information } \\
\hline Type & $n$ & Hearing Status $^{\mathrm{b}}$ & $n$ & Communication & $n$ \\
\hline Any/All & $\overline{4}$ & Hearing & $\overline{6}$ & ASL Only & $\overline{1}$ \\
\hline Physician (MD) & 5 & Non-Hearing & 2 & $\mathrm{ASL}+$ Interpreter $^{\mathrm{c}}$ & 5 \\
\hline Undisclosed & 1 & Unknown & 2 & $\begin{array}{c}\text { ASL + English } \\
\text { English Only }\end{array}$ & $\begin{array}{l}3 \\
1\end{array}$ \\
\hline
\end{tabular}

Note. The total number of responses for each subheading is $10(N=10)$.

${ }^{a}$ One participant identified as biracial Native American/White.

${ }^{b}$ Responses contradict focus group discussions (Hearing $=10$; Non-Hearing $=0$ ).

${ }^{\mathrm{c}}$ One interpreter was an unlicensed family member. 


\section{Appendix J}

\section{Figure 5}

The Trust Kite: Themes

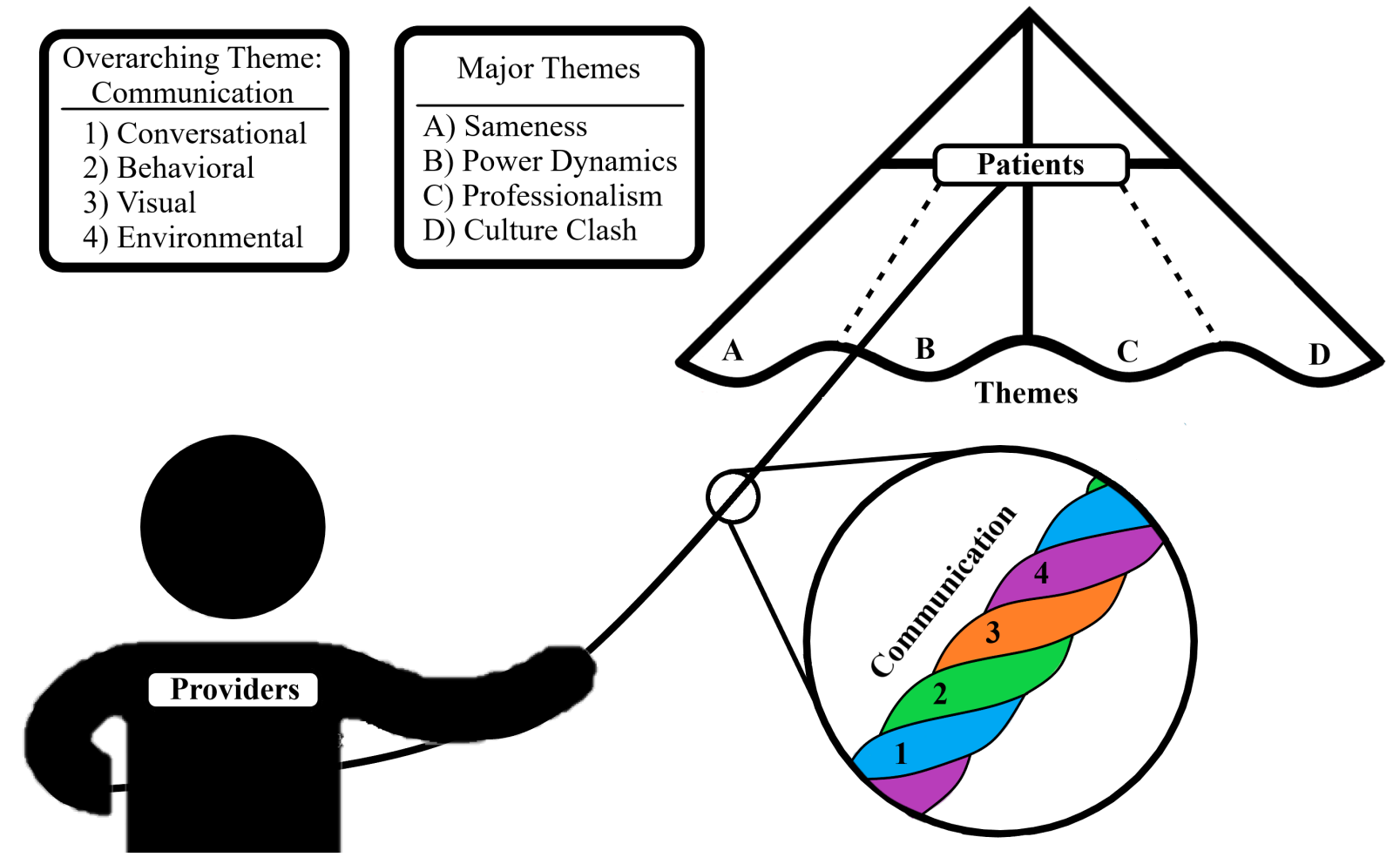




\section{Appendix K}

\section{Figure 6}

The Trust Kite: Trustworthiness

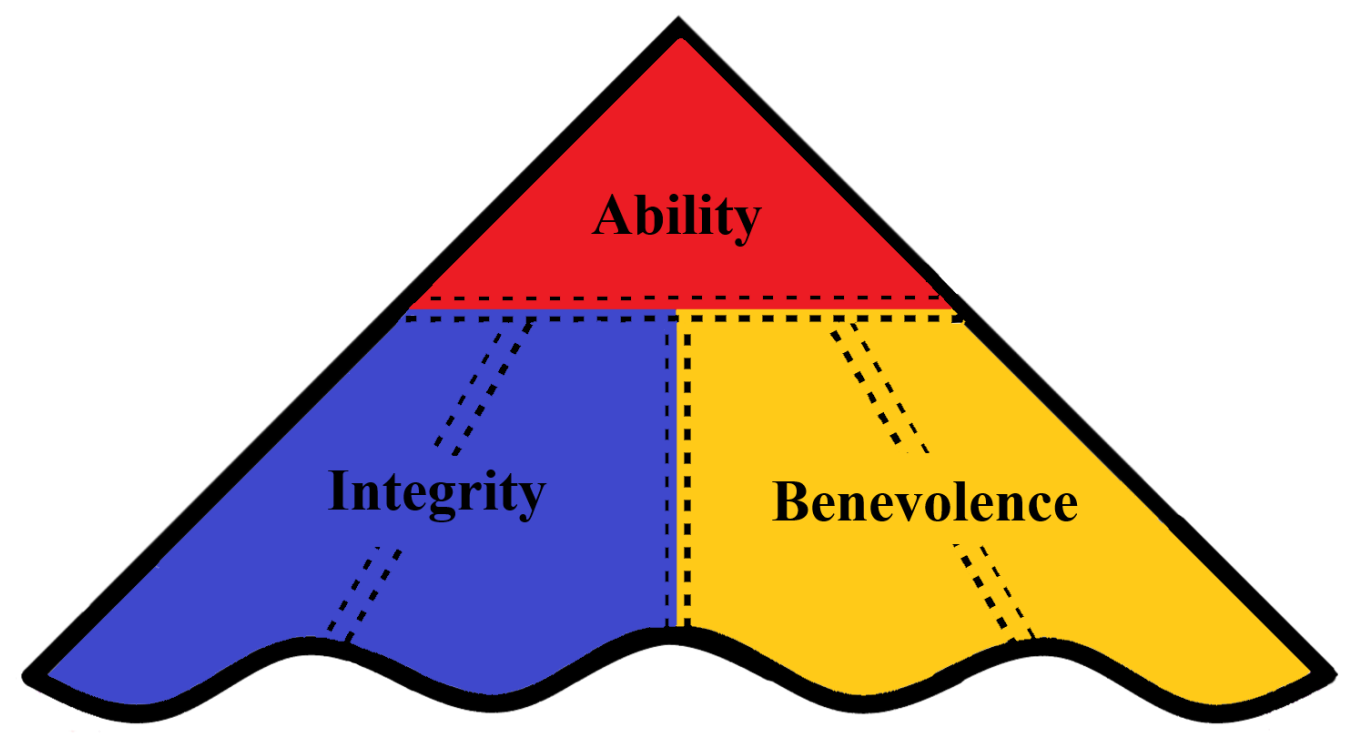

Note. The orientation of the themes (colors) in integrity and benevolence is arbitrary. See Appendix $\mathrm{J}$ for list of themes. 


\section{CURRICULUM VITAE}

NAME:

ADDRESS: $\quad$ University of Louisville, School of Nursing 555 S Floyd St.

Louisville, KY, 40202

EDUCATION: $\quad$ B.S., Human Biology: Health and Disease

Indiana University - Bloomington

2005-2010

B.S., Nursing (Accelerated)

Bellarmine University

2011-2012

MSN, Family Nurse Practitioner

Bellarmine University

2013-2015

Ph.D., Nursing

University of Louisville

2015-2020

EMPLOYMENT: Systems Nurse Educator, Norton Healthcare - Institute for Nursing Graduate Teaching/Research Assistant, UofL - School of Nursing Game Developer \& Facilitator, UofL - Thrivals and SHPEP

LICENSE \&

CERTIFICATIONS: Progressive Care Critical Nurse (PCCN)

Registered Nurse, Kentucky. (\#1151209)

Registered Nurse, Indiana. (\#28215406A)

Registered Nurse, North Dakota. (\#R37922)

2013-Present

2016-Present

2014

2012-2016

HONORS

\& AWARDS:

Jonas Scholarship

2016

Nurse of the Year (nomination)

PUBLICATIONS: Flynn, G.A.H., Polivka B., \& Behr, J.H. (2017). Smartphone use by nurses in acute-care settings (2017). CIN: Computers, Informatics, Nursing, 35(12). Published online December 2017. 
PRESENTATIONS: Flynn, G. A. H., Polivka, B. (2018). Data use and interpretation from a bedside nurse perspective - Keynote Speaker.

Research!Louisville: Hurstbourne Green. Louisville, KY

Flynn, G. A. H. (2018). Examples of Undergraduate Involvement in Research - Invited Podium Presentation. Research Symposium: University of Louisville, School of Nursing

Flynn, G. A. H. (2017). Best Practices in Handoff Reporting Podium Presentation. Motivational Magic: A PCA Experience Symposium: Norton Healthcare. Louisville, KY.

Flynn, G. A. H. (2016). Smartphone Use by Nurses in the Clinical Setting - Podium \& Poster presentation.

Research!Louisville: Baptist East Hospital. Louisville, KY

Flynn, G. A. H. (2015). Rapid Response Relevance: When to call and what to do. Grand Round Symposium: Audubon Hospital. Louisville, KY.

Flynn, G. A. H. (2014). Nursing tools: Don't be one, use one. Grand Round Symposium: Audubon Hospital. Louisville, KY.

Flynn, G. A. H. (2009). Carbon footprint calculations. National Teach-in on Global Warming. Bloomington, IN.

PRESS

INVOLVMENT: Kobin, B. (2020, Mar. 6). As coronavirus spreads, U of L students use board game to teach youth about pandemics. Courier Journal. Retrieved from https://www.courierjournal.com/story/news/education/2020/03/06/coronaviruslouisville-students-use-board-game-teach-pandemics/4975980002/

University of Louisville (2018, Apr. 11). UofL Today with Mark Hebert: Child birth dangers; Black males and mental health; Nursing Scholars [Audio Podcast]. Retrieved from $\underline{\text { https://soundcloud.com }}$

University of Louisville (2018, Jan. 15). UofL Today with Mark Hebert: NSA cybersecurity grant; Nurses and Smartphones [Audio Podcast]. Retrieved from https://soundcloud.com

PROFESSIONAL INVOVLEMENT: American Academy of Nursing Midwest Nurses Research Society 